\title{
SPATIO-TEMPORAL ACQUISITION, RECONSTRUCTION, AND VISUALIZATION OF DYNAMIC SCENES FOR PLANT PHENOTYPING
}

\author{
A Dissertation presented to \\ the Faculty of the Graduate School \\ at the University of Missouri-Columbia
}

In Partial Fulfillment

Of the Requirements for the Degree

Doctor of Philosophy

by

Ali Shafiekhani

Dr. Guilherme N. DeSouza, Dissertation Supervisor

December 2020 
The undersigned, appointed by the dean of the Graduate School, have examined the dissertation entitled

SPATIO-TEMPORAL ACQUISITION, RECONSTRUCTION, AND VISUALIZATION OF DYNAMIC SCENES FOR PLANT PHENOTYPING

Presented by Ali Shafiekhani, a candidate for the degree of Doctor of Philosophy, and hereby certify that, in their opinion, it is worthy of acceptance.

Dr. Guilherme N. DeSouza, Associate Professor, Dept. of Electrical Engineering and Computer Science

Dr. Jeffrey Uhlmann, Associate Professor, Dept. of Electrical Engineering and Computer Science

Dr. Kannappan Palaniappan, Professor, Dept. of Electrical Engineering and Computer Science

Dr. Felix Fritschi, Professor, Division of Plant Sciences 
Dedicated to my mother and father who taught me how to believe in myself, to pursue my dreams, and work hard for my destiny. I would not have got where I am today without their continuos love and support. 


\section{ACKNOWLEDGMENTS}

First and foremost, I wish to thank my advisor, Dr. Guilherme DeSouza who helped me along the way on this journey, and without his guidance, I would not have made it. His continuous support paved the way for me, and here I would like to extend my sincere gratitude to him. I also would like to express my deepest appreciation to my thesis committee: Dr. Jeffrey Uhlmann, Dr. Kannappan Palaniappan, and Dr. Felix Fritschi, for their valuable advice and practical suggestions.

I am deeply indebted to my family for all their love and support. Their trust and encouragement have made me strong not to fear of any problems. Many thanks to my parents, who helped me to pursue my dreams. Special thanks to my dear sister, Dr. Soraya Shafiekhani, who was beside me whenever I need her. I can not thank enough to my darlings Somayeh and Masoumeh who everything I have is because of them and my brothers, Hamid and Reza who are my true friends.

Last but not least, I would like to acknowledge and thank my lab mates, Dr. Luis Rivera, Tushar Nakini, Jacket Dembys, and Yixiang Gao, for their help throughout my study. 


\section{CONTENTS}

ACKNOWLEDGMENTS ....................... ii

LIST OF FIGURES . . . . . . . . . . . . . . . . . vi

LIST OF TABLES . . . . . . . . . . . . . . . . . $\mathrm{x}$

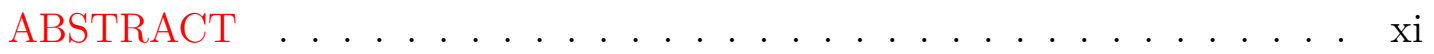

1 Introduction 1

2 Application, Material and Motivation 4

2.1 Platforms for Phenotyping . . . . . . . . . . . . 5

2.2 Computer Vision in Plant Phenotyping . . . . . . . . . . . . . 7

2.3 Developed Phenotyping System . . . . . . . . . . . . . . 7

2.3.1 Vinoculer ................... 8

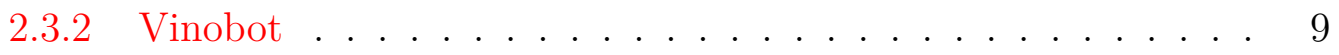

2.3.3 Advantages over Other Systems . . . . . . . . . . . . . . 10

2.4 Plants Behavior to Environment . . . . . . . . . . . . . . . 12

3 Challenges in Dynamic Scene 13

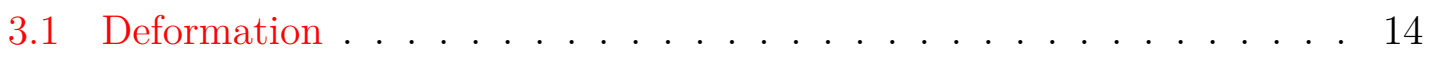

3.2 Motion ....................... 16

4 Literature Review $\quad 18$

4.1 Registration . . . . . . . . . . . . . . . . 19

4.2 SLAM or SFM . . . . . . . . . . . . . . . . . . . 19

4.2.1 Bayes Filter Framework . . . . . . . . . . . . . . 20

4.2.1.1 Parametric Filters . . . . . . . . . . . . . 21

4.2.1.2 Non-parametric Filters . . . . . . . . . . . . 24

4.2.2 Graph Based Framework . . . . . . . . . . . . . . . . 24 
4.2 .3 Bundle Adjustment . . . . . . . . . . . . . . . . . . . 26

5 Proposed Method $\quad 27$

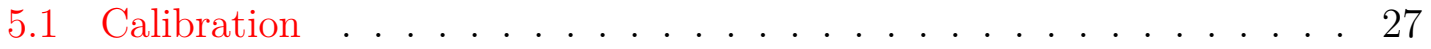

5.1.1 Geometric Camera Calibration . . . . . . . . . 27

5.1 .2 Stereo Camera Calibration . . . . . . . . . . . . . 28

5.1 .3 Head-eye Calibration . . . . . . . . . . . . . . . . 28

5.25 D Reconstruction Pipeline . . . . . . . . . . . . . . . 30

5.2 .1 Pre-processing . . . . . . . . . . . . . . . . . . 30

5.2 .2 3D Reconstruction . . . . . . . . . . . . . . 31

5.2.3 4D Reconstruction (Temperature) . . . . . . . . . . . . 32

5.2.4 5D Registration (Temporal) . . . . . . . . . . . 33

5.3 Non-Rigid Registration . . . . . . . . . . . . . . . . . . 36

5.3.1 Non-Isometric Transformation . . . . . . . . . . . . . . 36

5.3.2 Trust Region and Damped Methods . . . . . . . . . . . . . 37

5.4 VisND: a High Dimensional Spatio-Temporal Visualization Tool . . . 38

6 Experiments and Results $\quad 42$

6.1 Datasets . . . . . . . . . . . . . . . . . . . . . 42

6.1.1 Vinoculer (Temporal Stereo RGB-Thermal Images) . . . . . . 42

6.1 .2 Synthetic range data . . . . . . . . . . . . . . . 43

6.1.3 African violet spatio-temporal dataset . . . . . . . . . . 44

6.2 5D Reconstruction Pipeline . . . . . . . . . . . . . . 45

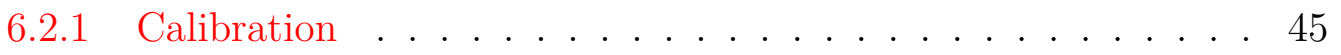

6.2.2 3D Reconstruction . . . . . . . . . . . . . 47

6.2.3 Validation of Thermal to 3D projection . . . . . . . . . 50

6.2.4 4D-RGB Point Cloud of the Canopy . . . . . . . . . . . . 51

6.2 .5 5D Reconstruction . . . . . . . . . . . . . 53

6.3 Non-Isometric Registration . . . . . . . . . . . . . . . . . . . . 54

6.3 .1 Synthetic range dataset . . . . . . . . . . . . . . 54

6.3.2 Non-Isometric Registration of African Violet dataset . . . . 56 
7 Conclusions and Future Work $\quad 65$

7.1 Future work . . . . . . . . . . . . . . 66

$\begin{array}{ll}\text { Bibliography } & 67\end{array}$

Vita

74 


\section{LIST OF FIGURES}

2.1 The developed platforms for high-throughput phenotyping in the field deployed at the Bradford Research Facility: (a) observation tower, Vinoculer (ViGIR-lab Phenotyping Trinocular Observer) shown at $15 f t$ high; and (b) ground vehicle, Vinobot (ViGIR-Lab Phenotyping Robot) ...................... 8

3.1 An agricultural field over the growing season (DAP: Days After Plant-

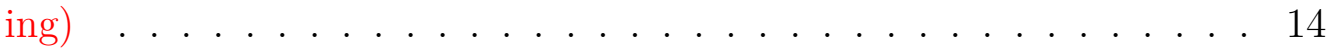

3.2 Temporal feature matching. Most mismatching are among keypoints that belong to the canopy . . . . . . . . . . . . . . 15

3.3 Temporal registration of two sparse point clouds created using images taken with 5 days time period gap . . . . . . . . . . . . 15

3.4 Variation of light condition over time . . . . . . . . . . . 16

4.1 Pose graph representation of SLAM. Blue nodes are pose of robots at different time, green nodes are the position of landmarks, and arrows are different type of constraints (observations) between nodes. . . . . 26

5.1 Three typical images of the aluminum + paper pattern used for calibration of RGB and IR cameras. (a) Left RGB; (b) IR Image; (c) Right RGB ..................... . . 29

5.2 Coordinate frame of left camera with respect to turn-table (head-eye

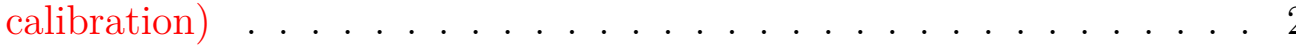

5.3 Depiction of the (a) spatial and (b) temporal constraints used for the creating of the 5D-RGB models . . . . . . . . . . . . 34

5.4 Temporal feature matching between images taken 5 days apart . . . . 35 
5.5 Effect of robot penalty function (a) Geman-McClure penalty function for different value of $\mu$,(b) The object function with Geman-McClure robust estimator as the penalty function [1]. . . . . . . . . . . . . 38

5.6 VisND Graphical User Interface (GUI) . . . . . . . . . . . . . . . 39

5.7 Snapshots of VisND demonstrating its current feature: (a) Manual ground detection (b) Plant segmentation by removing ground plane (c) Appearance rendering (d) Temperature rendering using Jet color

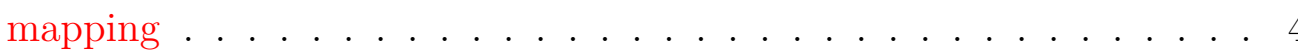

6.1 Field configuration at the MU Bradford Research Center. Small vertical boxes represent 10 plants, with sorghum marked by blue and corn marked by red boxes. Full boxes indicated plants selected for destructive sampling. . . . . . . . . . . . . . . . . . . . . 43

6.2 Synthetic range data . . . . . . . . . . . . . . . . . . . 44

6.3 (Left) Schematic representation of the 4D Scanner equipped with a stereo-rig camera, a turn table, two raspberry pi, and a LED lamp. It can collect temporal $360^{\circ}$ view of the object. (Right) A sample stereo images: top, and bottom images captured by 4D Scanner . . . . . . . 45

6.4 A time-series of 3D point clouds of the same African violet plant captured during the blooming of its flowers processed using COLMAP $[2,3]$. The proposed registration algorithm is able to capture its anisotropic growth, development and deformations over a 10-days period of time. For different days, the presented clouds are obtained from raw 2D stereo images taken exactly at the same time 01:00 AM. 45

6.5 Stereo RGB-Thermal camera configuration . . . . . . . . . . . . 46

6.6 Re-projections of the 3D coordinates of the corners obtained from left-right stereo reconstruction onto the thermal images. The " $x$ " and "+" indicate the re-projections and extracted corners from the thermal image, respectively. The images were not used in calibration

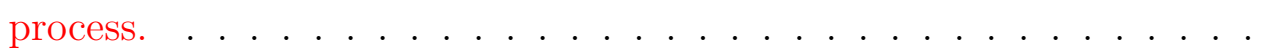

6.7 Re-projection error of 3D points found by triangulation of corners on left and right RGB images .................. . . . 
6.8 Configuration of selected points as collar of top most leaf on 3D model created by Vinoculer images . . . . . . . . . . . . . . . . . . . . . 48

6.9 3D Reconstruction of entire field (side view) using stereo RGB images collected by the Vinoculer . . . . . . . . . . . . . . . . . . . 48

6.10 Evaluation of phenotyping features: (a) height, and (b) Leaf Area Index (LAI) extracted from 3D models . . . . . . . . . . . . . .

6.11 (a) RGB(left) - Thermal (right) 3D model used for quantitative evaluation of thermal 3D projection. (b) Temperature of distinct points (in RGB and Thermal images) visible in point cloud and thermal image are compared. . . . . . . . . . . . . . . . 50

6.12 (a) RGB (top) Thermal (bottom) 4D reconstructed point cloud of the entire field at 19 DAP (days after planting), (b) Canopy temperature by ground subtraction . . . . . . . . . . . . . . 51

6.14 Snapshot of Thermal 3D models of the field at different times at 38

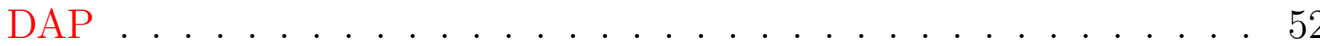

6.13 Snapshot of 4D-RGB models of the field throughout the season at

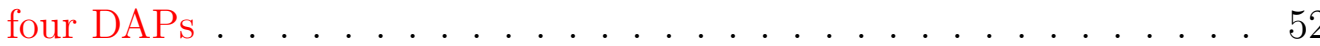

6.15 Temporal registration of two sparse point clouds created using images taken with 5 days time period gap (a) before optimization (b) after

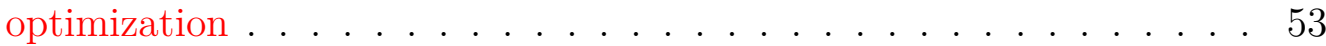

6.16 Non-rigid registration for different $\mu$ values of Geman-McClure robust

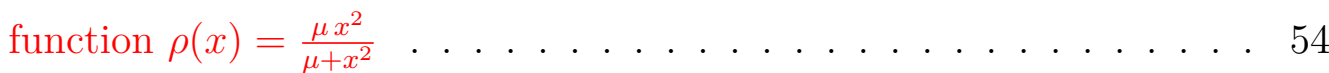

6.17 Optimization of non-rigid registration using Geman-McClure robust estimator objective function . . . . . . . . . . . . . . 55

6.18 A comparison between objective functions defined (a) with penalty function, (b) without penalty function, (c) as described in fast global registration approach $[1] \ldots \ldots \ldots \ldots$. . . . . . . . . 56

6.19 Qualitative results for non-rigid registration. Top row models show source and target point cloud before registration. Middle and bottom rows are the results of non-rigid registration for models with no noise and noise level 2 , respectively. . . . . . . . . . . . . . 57 
6.20 The pipeline for the proposed method of spatio-temporal reconstruction 57

6.21 An example of the 3D registration of the same two consecutive point clouds from the violet dataset using (a) PFH, (b) FPFH, and (c)

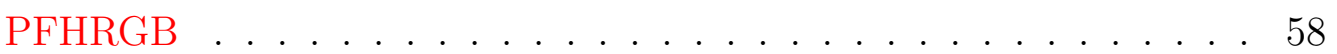

6.22 Representation of bundles with sliding window and gap. At each bundle optimization, all models within the bundle are aligned with respect to the first model. Sliding this bundle optimization by a gap step smaller than window size ensures overlaps. . . . . . . . . . . 60

6.23 registration of violet dataset using the pairwise approach. . . . . . . 61

6.24 registration of violet dataset using the incremental approach. . . . . 62

6.25 registration of violet dataset using the bundle approach. . . . . . . . 63

6.26 A comparison between all the registration methods over 10 days under the 7 DoF transformation . . . . . . . . . . . . . . . 64

6.27 Snapshots of VisND with Spatio-temporal models of African violet . . 64 


\section{LIST OF TABLES}

2.1 Comparison between Vinoculer and the UAV-based phenotyping system presented in [4]. . . . . . . . . . . . . . . . . . . 11

2.2 Comparison between field-based systems that perform phenotyping

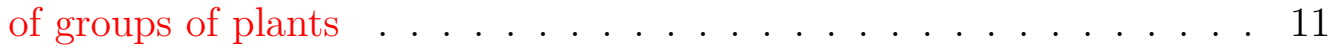

2.3 Comparison between field-based systems that perform detailed phenotyping of individual plants . . . . . . . . . . . . . . . . . 12

6.1 Sampling method performed per indicated type of plots . . . . . . . . . 43

6.2 Average errors for each time-series registration approach with theAfrican violet dataset under 6, 7 and 9 DoF transformations. The values in the table are expressed in millimeter $(\mathrm{mm})$. . . . . . . . . . 63 


\begin{abstract}
Recent advances in robotic vision are permitting breakthrough solutions to various agriculture-related problems, including farming, field phenotyping, food security, etc. Among these technological advancements, autonomous data collection for field phenotyping enables plant scientists to further correlate plant behavior to environmental conditions using a large amount of high-quality data captured throughout the growing season. In that sense, the ability to process such a large amount of image data must be made possible via computer vision techniques that fuse raw data in order to extract relevant changes during the growing season. For instance, correlating 3D models of plants over time enables scientists to analyze phenotypical data and identify favorable traits in terms of maximum growth, yielding, response to abiotic stressors, etc. Furthermore, the fusion of 3D models of individual plants into larger and cohesive models of the entire crop over time can lead to important discoveries in plant genomics by biology researchers. In this work, we propose an end-to-end system to acquire, fuse, and visualize multimodal data collected over time from an agricultural field. The system consists of two field robots and a visualization tool. The robots can collect and process RGB, Thermal, and 3D information from close proximity or from above ground. The visualization tool allows users to select and process the same data in order to create Spatio-temporal, high dimensional models that describe the physical and temporal structure, color, and temperature of plants in crop fields. Once created, users can visualize the same N-dimensional models using a newly proposed GUI, named VisND. Technically speaking, the proposed system registers time-stamped 5D models (3D plus RGB and IR) of the field as observed by the robots into a single-registered Spatio-temporal model. This process requires a non-rigid (ie. deformable) registration of the $3 \mathrm{D}$ structures so that plant
\end{abstract}


behavior (e.g. growth, leaf angle, response to stress, etc) can be visualized over time. 


\section{Chapter 1}

\section{Introduction}

In the past years, computer vision problems have found applications in different industries e.g. medicine, agriculture, transportation, military to name few. These methods are often required to be customized for the used case, make use of assumptions to simplify the problem, add more or relax some constraints that exist in the application. For instance, while most computer vision algorithms make use of the static scene assumption, a lot of real-world problems have to deal with dynamic scenes. In such cases, temporal change is the key information to be extracted from the problem. Therefore, one should revisit the problem and take into account temporal information.

In agriculture, monitoring plants' behavior and their response to environmental conditions is called plant phenotyping. Plant phenotyping helps scientists to better understand the crops and allows them to link different genotypes with a particular response. Monitoring the plants over the growing season and collecting informative data have attracted great attention past few years. While traditional methods used manual data collection, different automated systems have recently been introduced to collect detailed data. These systems can be classified into two groups: indoor (greenhouse), and outdoor (field). Although, greenhouses offer extraction of a large number of features throughout the year, correlation of these features with those of plants grown in the field is often unsatisfactory. On the other hand, a system that can collect and extract a large amount of data from crops in the field faces more challenges such as weather conditions, unstructured crop, and field configuration. 


\section{Chapter 1. Introduction}

Another challenging problem one has to deal with is the temporal changes that happen in the field as plants grow. Having a dynamic scene adds a level of complexity to the problem specially if the temporal information is required. There are cases that the problem is solved by ignoring the dynamic components of the scene as outliers. In such cases, although the algorithm is robust even in presence of moving or deformable objects, one could not extract temporal information.

3D Modeling is active research in computer vision and has applications in various fields including agriculture. For instance, correlating 3D models of plants over time enables scientists to analyze phenotypical data and identify favorable traits in terms of maximum growth, yielding, response to abiotic stressors, etc. Furthermore, the fusion of $3 \mathrm{D}$ models of individual plants into larger and cohesive models of the entire crop over time can lead to important discoveries in plant genomics by biology researchers. A combination of RGB, Thermal and 3D model over the growing season makes the interpretation of plant status in the context of environmental conditions easier.

In this work, we developed two phenotyping platforms for the in-situ characterization of plant responses to changes in their environment. These platforms consist of two robotic systems: a mobile, observation tower, for canopy characterization and general inspection of the crop; and a ground vehicle, for individual plant phenotyping. Together, the developed platforms can collect data in two scales: an entire field and individual plants which makes the architecture a suitable system for high throughput plant phenotyping. Our developed phenotyping platforms can collect multi-modal spatio-temporal information from plants and process the raw data and create unified and consistent data that can be used to extract phenotyping features. Specifically, we developed a pipeline to create time-stamped 5D models (3D plus RGB and IR) of the field. The spatio-temporal model is capable of providing rich information such that many plant phenotypes can be extracted and correlated with each other and with plant performance. The spatio-temporal reconstruction pipeline is as follows: first 3D models of the canopy at different timestamps are reconstructed, next, temperature information is fused with the reconstructed 3D model and finally, all individual temporal models are registered to the same refer- 


\section{Chapter 1. Introduction}

ence coordinate frame. This step requires a non-rigid (ie. deformable) registration of the 3D structures so that plant behavior (e.g. growth, leaf angle, response to stress, etc) can be visualized over time.

Plant phenotyping is data-driven research where the interpretation of large and often multidimensional data is required. Therefore, effective visualization of plant phenotypes plays a major role in data analytics as it can provide scientists with the required tool to extract and infer important information. In that sense, unifying the large and multidimensional phenotypical data into one single model of the canopy can help plant biologists to correlate information from different dimensions and derive new observations and understandings in plant sciences. Therefore, we developed a spatio-temporal visualization tool for high-dimensional temporal visualization of canopy for plant phenotyping. The goal is to offer an open-source visualization tool, named VisND (for N-Dimensional), that will provide a Graphical User Interface (GUI) where plant scientists can easily extract and analyze multidimensional models, registered over time, from different sensors and viewpoints.

The rest of this work is organized as follows: in Chapter 2, an overview of the application i.e. plant phenotyping is given and the developed material to solve the problem is introduced. Chapter 3 reveals the challenges one could face when dealing with a dynamic environment and identifies places in the algorithm where can be affected by the dynamic environment. Chapter 4 presents the literature of computer vision problems e.g. registration and reconstruction and identifies the strength and weaknesses of them. In Chapter 5 the proposed method for spatiotemporal reconstruction and visualization of the agricultural field is described in detail. Chapter 6 illustrates experiments and results obtained from our developed hardware and software plant phenotyping system. Finally, in Chapter 7 we conclude our work and present future work considered in the study. 


\section{Chapter 2}

\section{Application, Material and Motivation}

Population increases, climate change, degradation and loss of arable land, and the increasing appearance of new pests and diseases threaten the world's food supply [5]. Understanding how plants respond to environmental and genetic perturbations is essential to accelerating the improvement of crops and agriculture [6]. High-throughput phenotyping provides an unprecedented opportunity to study the physiological, developmental, and molecular mechanisms that govern the dynamic behavior of plants [7]. However, existing systems that allow the highly automated collection of basic phenotypic data for small numbers of plants in the greenhouse fall far short of the need to examine and characterize thousands of plants under real world conditions. Building systems that can collect multi-modal, multi-character data in real time in the field requires integrating plant biology and crop science with robotic vision and computer engineering. These systems must be accurate and reliable, and should provide richer information than the current methods available for automated greenhouse or manual field phenotyping. By doing so, they will help us to link plant genotypes as well as the molecular and eco-physiological responses with the expression of specific phenotypes in response to the growing conditions [8].

In recent years, given the world requirements for high-yielding crops, demand for more fully automated systems is increasing. Those systems include both manned and unmanned, as well as ground and aerial vehicles, all equipped with advanced 


\subsection{Platforms for Phenotyping}

sensors and sophisticated control algorithms. From those early systems to today's field robotics, developing reliable sensing technologies, from reliable odometry to sophisticated machine vision, plays a key role in advancing agricultural automation.

\subsection{Platforms for Phenotyping}

Crop canopy characteristics critically influence yield formation [9]. In fact, important traits such as plant height, weight, volume, biomass, shape, color, temperature, light absorption, and potentially many others can be obtained from the simple observation of the canopy as a whole. In that case, thermal, multi-spectral, and hyper-spectral imaging from either airborne or remote sensing (satellite) can play a greater role in plant phenotyping in the field. Unfortunately, the cost and availability of these systems may pose a large burden on research and still lack the necessary resolution. In that sense, while some researchers invest in larger vehicles to increase payload, optimize volume and type of data acquired on a single flight [4], other systems rely on multiple micro-UAVs to achieve higher availability [10] and still perform canopy characterization. In [11], for example, a simple, but an ingenious technique for measuring the height of plants using micro-UAVs was proposed. In that system, a laser scanner was mounted onto the micro-UAV to estimate plant height by measuring the difference between the ground and the top of the canopy.

Based on the literature, platforms that are designed for HTPP can be grouped into two categories: those developed for indoor (greenhouse or laboratory) [12] and those designed for outdoor environments (field) $[13,14]$. Alternatively, platforms may be classified based on whether they collect data from a group of plants or from individual plants.

The majority of efforts in recent years has been placed on the development of HTPP platforms for plants grown in controlled environments (i.e. greenhouses). For instance, in [12], the authors introduced an automated HTPP platform, Phenoscope, to collect data from 735 individual pots brought into imaging stations by conveyor belts. Like other indoor platforms, Phenoscope is able to water, weigh and image plants continuously, allowing for homogenized conditions for all plants. The same 


\subsection{Platforms for Phenotyping}

is true for the Scanalyzer ${ }^{3 D H T}$, made by LemnaTec and reported in [15]. However, this indoor platform also has the ability to capture images in different wavelengths: i.e. from far infrared (FIR) to ultraviolet (UV). The platform is equipped with a system of conveyor belts capable of carrying 600 individual plants, and its imaging systems can capture 9000 images per day [15]. Each plant carries a radio frequency identification (RFID) for individualized data management, watering and nutrient supplementation. Although, greenhouses offer extraction of a large number of features throughout the year, correlation of these features with those of plants grown in the field [16] [17] is often unsatisfactory.

Therefore, platforms for field phenotyping with various degrees of automation have emerged over the years $[13,14,18-21]$. In [19], for example, the authors introduced a tractor-pulled platform, BreedVision, consisting of various optical sensors, such as light curtain, 3D Time-of-Flight (ToF) cameras, laser range sensors, and multispectral cameras, including RGB. A similar platform was recently presented in [14], with a series of sensors including infrared thermometer (IRT), ultrasonic sensor, and crop canopy sensors: multispectral, NDVI, etc. This combination of sensors allowed the system to collect both architectural and morphological information from plants. However, they employed a vehicle with limited clearance, and hence, limitations on the types of plants to be analyzed could be expected. Also, while the system could cover a reasonably large area at each passage, its limited maneuverability and the fact that navigation was not fully autonomous constrained its throughput and its ability to freely move in the field. In that sense, a faster and more flexible approach is found in [20], thanks to its more autonomous field navigation and its individual plant phenotyping capabilities. Adapting existing equipment is not always a limiting factor. In [13] for example, a field-based HTPP platform was developed using a high-clearance tractor - later referred to as a Phenomobile [22]. Three types of sensors were grouped in four sets and mounted on the front boom of the tractor enabling the simultaneous collection of data from four adjacent rows of cotton plants. Although most phenomobile platforms have a high coverage rate (in this case, $0.84 \mathrm{ha} / \mathrm{h}$ ), soil compaction is often a main concern [23]. 


\subsection{Computer Vision in Plant Phenotyping}

In order to understand plant adaptation to the environment and management practices, architectural phenotypes such as height, width, leaf area, leaf angle, leaf color, etc. are very important [24]. Traditionally, these traits are measured by hand, consuming an enormous amount of time. To date, computer vision has already made an impact in speed and volume of plants phenotyped (i.e. high throughput), specially when it comes to phenotyping in growth chambers and greenhouses [12, 16, 25-28] [29]. Also, 3D imaging of plant shoots and roots [30-32] are becoming the standard in storing all possible details from plants - i.e. details with hitherto unknown value, but which can prove useful in the future. Indeed, while 3D imaging in the field may still have a long way to go vis-a-vis their greenhouse counterparts, some systems, such as in [33], are already causing great impact.

\subsection{Developed Phenotyping System}

As evidenced by previous sections, advances in sensing technology can make plant phenotyping more reliable, easily available, and accurate. Consequently, robots that can deliver and operate those sensor platforms in the field are in high demand. In fact, without robots that can operate such sensors automatically and with high availability in collection of both structural and environmental characteristics, true HTPP is impossible. So, in our research, we developed two phenotyping platforms [34, 35] for in-situ characterization of plant responses to changes in their environment (e.g., management practices, drought, flood, heat, etc.). These platforms consist of two robotic systems: a mobile, observation tower, Vinoculer, for canopy characterization and general inspection of the crop; and a ground vehicle, Vinobot, for individual plant phenotyping. Figure 2.1 shows the Vinobot and Vinoculer in a field at the Bradford Research Center near Columbia, Missouri, USA.

The first platform, Vinoculer, for ViGIR-Lab (Vision-Guided and Intelligent Robotics Lab, Columbia, MO, USA) Phenotyping Trinocular Observer, is a mobile observation tower equipped with a 360-degree robotic vision system that can oversee a large area of the crop using two (stereo) RGB cameras and one IR (thermal) 


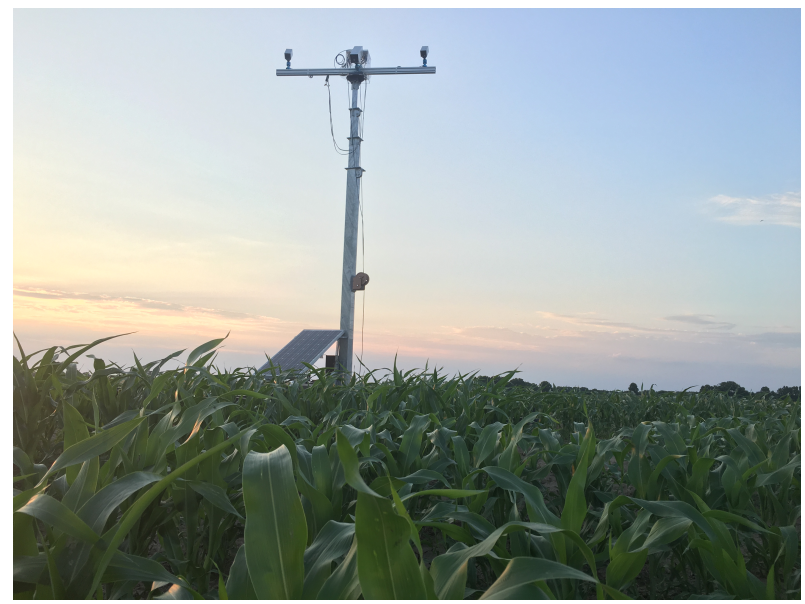

(a) Vinoculer

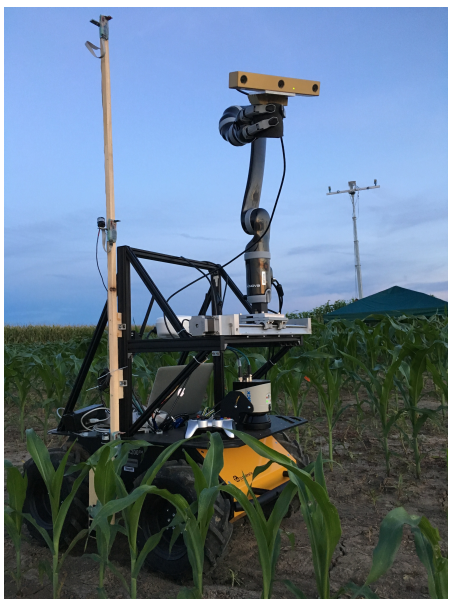

(b) Vinobot

Figure 2.1: The developed platforms for high-throughput phenotyping in the field deployed at the Bradford Research Facility: (a) observation tower, Vinoculer (ViGIRlab Phenotyping Trinocular Observer) shown at 15ft high; and (b) ground vehicle, Vinobot (ViGIR-Lab Phenotyping $\overline{\text { Robot) }}$

camera. The main purpose of this system is to detect regions of the crop under stress, and deploy the ground vehicle for further investigation.

The ground vehicle, or Vinobot for ViGIR-Lab Phenotyping Robot, is responsible for phenotyping plants individually. That is, the Vinobot moves around the field and collects data from each plant, on a regular schedule or by demand. The Vinobot consists of multiple sensors, such as 4D imaging (3D stereo + thermal), temperature, humidity, light intensity (PAR), etc. On the Vinobot, we also included a differential GPS, a robotic arm, a LIDAR, and other support equipment for autonomous navigation and operation of the phenotyping sensors.

\subsubsection{Vinoculer}

Vinoculer is a portable observation tower mounted in the center of a field. It is capable of turning 360 degrees while capturing data from a large area. It was equipped with stereo RGB and IR cameras in order to perform measurements such as volume, leaf area, biomass, height, growth rate and other canopy characteristics. Together, the Vinobot and Vinoculer are capable of collecting data from a large area at the canopy and individual plant level, as will be explained later.

The Vinoculer was equipped with two RGB-spectrum cameras for stereo vision 


\subsection{Developed Phenotyping System}

(Grasshopper3 by PointGray), an IR camera (Flir A625), a 360 turntable with an accuracy of $0.1^{\circ}$, a temperature sensor, and WiFi. This equipment was mounted on a telescopic and easily movable tower, which could be elevated to heights in the range of $3 \mathrm{~m}$ to $10 \mathrm{~m}$. Given the characteristics of the current lenses $(8 \mathrm{~mm})$, the tower provided Vinoculer with a viewing area with $30 \mathrm{~m}$ diameter, when deployed at maximum height, but lenses with shorter focal lengths could be used to increase the viewing area.

The two 12Mpixel RGB cameras were placed on a horizontal bar to provide a stereo baseline of approximately $1.7 \mathrm{~m}$ - the IR camera was mounted at the center, between the two RGB cameras. The turntable rotated the set of cameras with respect to the base and the Vinoculer was programed to image the field at regular intervals - as often as every $5 \mathrm{~min}$ and in $0.1^{\circ}$ steps. Vinoculer V1 and V2 used two Raspberry-Pi's-V3 to control the turntable and the cameras and a total of 64 GB of memory stored data for up to 10 consecutive days. In the third version of Vinoculer, the computing unit was upgraded to NVIDIA Jetson TX-2 to process data on-the-fly. One temperature sensor was added to measure air temperature. The entire system was powered from the ground by $12 \mathrm{~V}$ lead-acid batteries, which were charged through solar panels, but could last 2 days without sunlight. The entire system was weatherized to tolerate typical summer conditions in the Midwest of the USA - i.e., over $100^{\circ} \mathrm{F}\left(37.8^{\circ} \mathrm{C}\right)$ temperatures, rain, and strong winds (50 mph or $22.3 \mathrm{~m} / \mathrm{s}$ on record for the period of deployment). The tower was also equiped with a broad range end-to-end WiFi to enable remote access to the on-board computer and upload data to the server.

\subsubsection{Vinobot}

As mentioned, Vinobot is a mobile platform equipped with a range of sensors designed to fulfill both the phenotyping tasks and its autonomous navigation needs. Figure 2.1b depicts the equipment installed aboard the Vinobot, which will be explained in detailed next.

Vinobot was implemented around the Husky A-200 from Clearpath. The hardware components of the Vinobot are shown in Figure 2. A linear slide at the front 


\subsection{Developed Phenotyping System}

of the robot guides a robotic arm (JACO2 from Kinova), which was mounted on the slide to improve lateral reach. The purpose of the robotic arm is to allow for multiple sensors to be handled by the robot. Currently, a stereo-thermal sensing unit containing BumbleBee XB3, by PtGray and A65 by Flir is handled by the arm to perform 4D imaging (3D-RGB-Thermal).

In terms of environment, three sets of temperature (TMP36), humidity (HIH-4030), and light intensity (TSL2561, LI-190 SA, and LI-200 SA) sensors were mounted along a vertical bar at three different heights. The three sets perform measurements close to the ground, at mid-plant height and near the top of the canopy. While the robotic arm could chose between multiple sensors and it could easily position a single set of environmental sensors at any height, the use of a vertical bar with the three sets already mounted at three heights was chosen to increase throughput. Also, in terms of light intensity, four sensors were added at the same three positions on the bar: a Pyranometer at the top and a Quantum at the mid-plant and ground levels; and a Luminosity and photo diode at all three levels.

Once again, the purpose of the robotic arm is to move the cameras around individual plants, allowing for multiple viewing angles and resolving any potential occlusions while imaging the plant. Various other equipments were contained within the Vinobot, including an on-board PC (Mini-ITX Single board), GPS (Novatel Smart6-L), LiDAR (Sick LMS 151), long-range WiFi, ADCs, Arduinos UNO, etc. These devices are mostly required for the navigation task, but they may also provide support for phenotyping.

\subsubsection{Advantages over Other Systems}

Our architectural design focused on maximizing accuracy, throughput, and payload, while minimizing cost and downtime. So here, we present a comparison between the components of the developed architecture and other systems in the literature. Our goal is to demonstrate that when accounting for all characteristics above, our system has clear advantages.

As mentioned earlier, one way of achieving a minimum throughput of hundreds of plants per hour to be considered a HTPP system [25] is to perform aerial imaging 
Table 2.1: Comparison between Vinoculer and the UAV-based phenotyping system presented in [4].

\begin{tabular}{|c|c|c|}
\hline Platform & Phenocopter [4] & Vinoculer \\
\hline \hline Payload & $1.5 \mathrm{Kg}$ & $10 \mathrm{Kg}$ \\
\hline Flight Time & $30 \mathrm{~min}$ & - \\
\hline Area/Flight & $3 \mathrm{ha}$ & - \\
\hline Availability & 7 flights/day & $24 / 7$ \\
\hline Total Area Covered & $21 \mathrm{ha} /$ day & $2.2 \mathrm{ha} /$ day \\
\hline Type of Camera & RGB, Thermal and NIR & Limited by payload \\
\hline Max wind speed & $11 \mathrm{~m} / \mathrm{s}$ & $22.3 \mathrm{~m} / \mathrm{s}^{1}$ \\
\hline
\end{tabular}

Table 2.2: Comparison between field-based systems that perform phenotyping of groups of plants

\begin{tabular}{|c|c|c|c|c|}
\hline Platforms & Vinobot & Vinoculer & Phenomobile [13] & Scanalyzer ${ }^{\text {Field }}[36]$ \\
\hline \hline Type & Semi-automated & Fully-automated & Manually-driven & Fully-automated \\
\hline Plants/h & 35430 & 12,648 & - & - \\
\hline ha/h & 0.41 & 0.09 & 0.84 & 0.002 \\
\hline Images $/ \mathrm{h}$ & 324000 & 2592 & - & 115 \\
\hline Bytes/h & $380 \mathrm{G}$ & $5.4 \mathrm{G}$ & $2.094 \mathrm{M}$ & $6.7 \mathrm{G}$ \\
\hline \multirow{3}{*}{ Capabilities } & $\begin{array}{c}\text { RGB, humidity } \\
\text { temperature } \\
\end{array}$ & $\begin{array}{c}\text { Mobile, Stereo RGB } \\
24 / 7, \text { IR imaging, } \\
\text { light intensity }\end{array}$ & $\begin{array}{c}\text { Air Temperature } \\
\text { sonar sensors } \\
\text { Multispectral }\end{array}$ & $\begin{array}{c}\text { RGB, Multispectral } \\
\text { Fluorescence }\end{array}$ \\
\hline
\end{tabular}

using UAVs. So, in this section we first compare the Vinoculer with a typical UAVbased system reported in [4]. Table 2.1 summarizes this comparison mainly in terms of payload, availability, total area covered, and max wind speeds. As the table shows, our system compares favorably in all but one of these criteria: total area covered.

Next, we considered typical field-based systems that perform phenotyping of groups of plants without resorting to UAVs. In this case we assumed both Vinoculer and Vinobot collect only groups of plants, and Table 2.2 summarizes the advantages of the combined platforms with respect to two other systems: the "Phenomobile" in [13] and the Scanalyzer Field in [36]. We call the attention of the reader to the fact that the combined Bytes/h of our architecture is comparable to the commercial Scanalyzer ${ }^{\text {Field }}$, for a much lower price and without the confinement of a 1000sqm area, since both of our platforms are mobile. 


\subsection{Plants Behavior to Environment}

Table 2.3: Comparison between field-based systems that perform detailed phenotyping of individual plants

\begin{tabular}{|c|c|c|c|}
\hline Platforms & Type & Plants/h & Images/h \\
\hline Vinobot & Outdoor/Semi-automated & 120 & 1440 \\
\hline Scanalyzer $^{3 D H T}[15]$ & Indoor/Fully-automated & 25 & 375 \\
\hline Phenoscope [12] & Indoor/Fully-automated & 185 & 3750 \\
\hline
\end{tabular}

Finally, in Table 2.3 we compare phenotyping platforms that can performed detailed, and hence time consuming scans of individual plants. Once again, while our platform performs below one of the systems in the literature, it performs much higher than the other system and it does so in outdoor conditions.

\subsection{Plants Behavior to Environment}

In order to understand plants behavior in response to environment, one should capture, measure, and track changes that happen over time for different plants (with different genotypes). Therefore, the system requires frequently collecting (plants can respond to changes within $30 \mathrm{~min}$ ) multi-modal data over a large area covering all the plants. However, the raw data collected with such frequency and scale is not helpful as one cannot directly infer important spatial and temporal features. The main goal of this work is to provide a pipeline to process the large amount of data collected by our proposed automated platform and create a consistent spatiotemporal model of plants that one can accurately extract phenotyping features. Indeed, such system along with the processing pipeline can provide high-throughput plant phenotyping in the field. 


\section{Chapter 3}

\section{Challenges in Dynamic Scene}

While most computer vision algorithms make use of the static scene assumption, a lot of real-world problems have to deal with dynamic scenes. The scene can become dynamic by various changes over time such as motion and deformation which can be happen slow or fast in time. Although other factors like changes in lighting, shading, and texture conditions over time can also change the appearance of the world in an image, here, they are not considered as the sources of being dynamic. In all these cases, time is an essential component to define the state of the world.

Almost all the real-world applications have some degrees of dynamics where the temporal information is often important. The agricultural fields can be considered as an example of a dynamic environment where the growth of plants is the source of changes. In this case, the deformation of objects (plants) is changing the appearance of the field as plants grow. The urban roads are another example of a dynamic environment where the movement of vehicles or pedestrians makes the scene dynamics. There are other applications like human body motion where both deformation and movement can cause temporal changes in appearance.

Having a dynamic scene adds a level of complexity to the problem especially if the temporal information is required. There are cases that the problem is solved by ignoring the dynamic components of the scene as outliers. Although in such cases the algorithm is robust even in presence of moving or deformable objects, one could not extract temporal information.

The state-of-the-art SLAM or SfM algorithms also assume that the scene is static 


\subsection{Deformation}

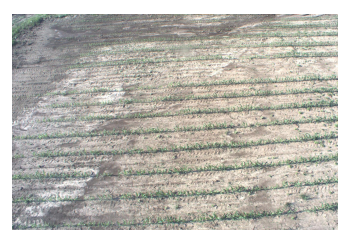

DAP 19

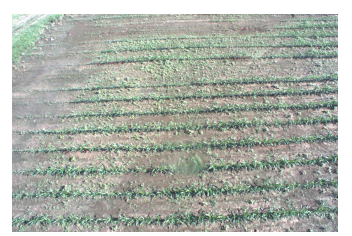

DAP 24

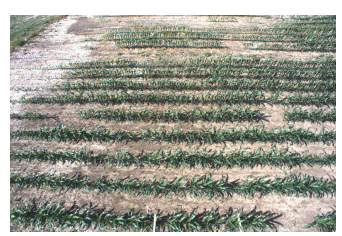

DAP 29

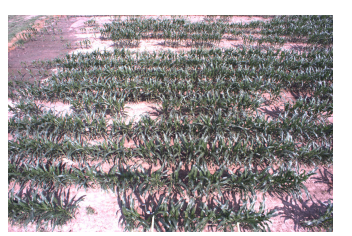

DAP 38

Figure 3.1: An agricultural field over the growing season (DAP: Days After Planting)

and objects are rigid with respect to the background. Although these algorithms can handle movement or deformation of objects to some extend by ignoring them as outliers, they can not capture motion information or temporal shape of moving or deformable objects, respectively.

In next sections, two source of dynamic scenes are explored: deformation of an object and non-rigid motion of objects.

\subsection{Deformation}

Non-rigid objects are commonly seen in our surroundings. They are challenging to deal with as their shapes can be changed over time. Depending on the application, there are different levels of complexity when it comes to non-rigid objects. The complexity varies from isometric scale deformation to arbitrary deformation of each point in space. The main challenge is the difficulty to model the geometry of a nonrigid object or a scene containing such an object. For example in Structure from Motion (SfM), the relative pose of two frames is calculated by reprojecting points of the object from one frame to another, however, when the object is non-rigid the corresponding points either cannot be found or the object geometry should also be taken into account to find the relative pose of two frames.

Figure 3.1 shows an agricultural field during the growing season. As can be seen, the appearance of the field is dramatically changing as plants grow. This potentially creates various difficulties if one tries to reconstruct the canopy both spatially and temporally.

First, point correspondence between temporal frames is challenging as the scene structure is constantly changing. Even scale-invariant feature descriptors like SIFT 


\subsection{Deformation}

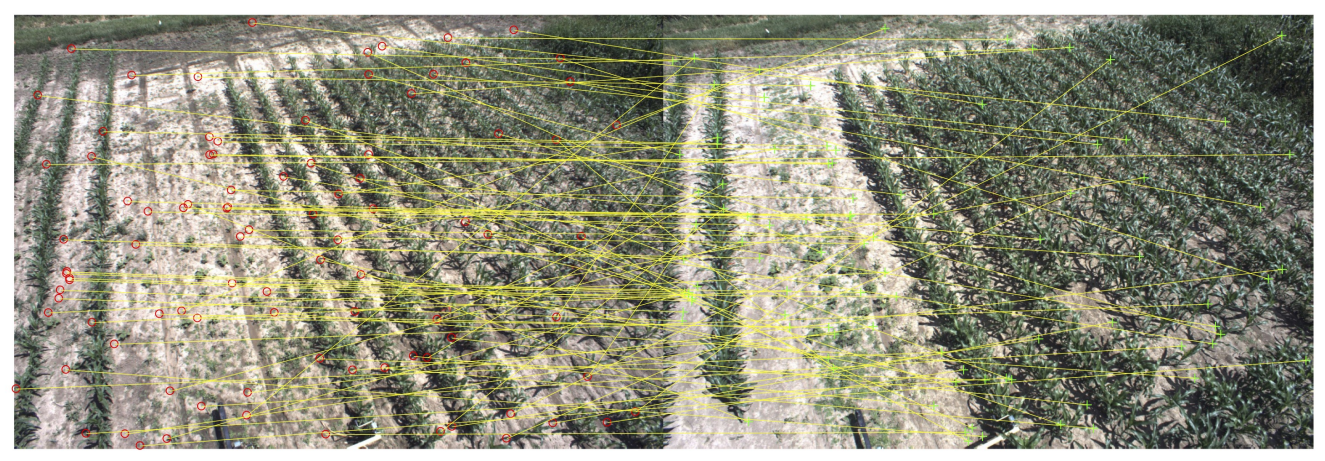

Figure 3.2: Temporal feature matching. Most mismatching are among keypoints that belong to the canopy

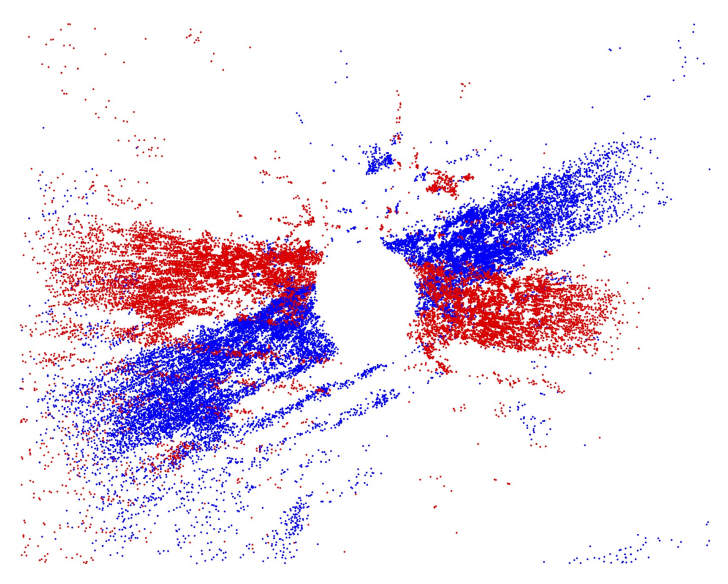

Figure 3.3: Temporal registration of two sparse point clouds created using images taken with 5 days time period gap

cannot be reliably matched for keypoints belong to deformable objects (plants).Figure 3.2 shows a typical SIFT feature matching over temporal frames captured 5 days apart. Most of the mismatches are among the keypoints that belong to the canopy.

Second, to create temporal models of growing plants one has to define temporal constraints to register temporal cloud of points. Given a deformable object like the canopy makes it extremely difficult to construct such constraints especially if the temporal gap is relatively large. Figure 3.3 shows two point clouds captured with 5 days time period gap. Registration of these two clouds of point using conventional rigid-body registration algorithms like ICP fails due to structural changes in the canopy.

Third, the variation of lighting condition especially over a long period (couple months) adds more complexity to the problem. Figure 3.4 shows typical images 


\subsection{Motion}

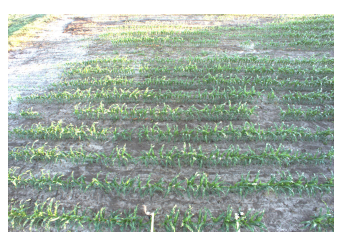

7:00 AM

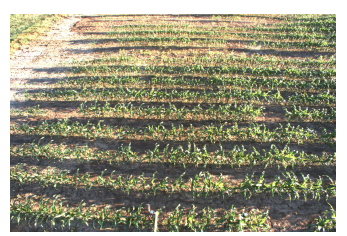

8:00 AM

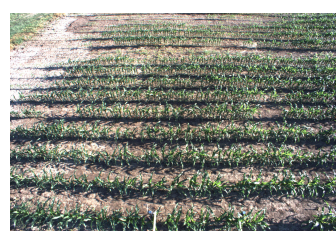

10:00 AM

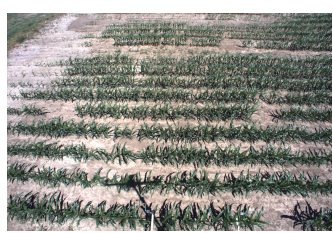

12:00 PM

Figure 3.4: Variation of light condition over time

of canopy captured within a couple of hours. As can be seen, the light condition for images taken even on the same day can be extremely varied which makes the temporal association harder.

\subsection{Motion}

The motion is another factor that leads to a dynamic scene. If one or more objects (e.g. leaves) independently move with respect to the background or each other then the state-of-the-art SfM or SLAM algorithms are unable to extract temporal information from the sequence of temporal images. Looking at the pipeline of these algorithms, one could see the footprint of static scene assumption in e.g. feature extraction and matching, 3D triangulation, relative pose estimation, loop closure and etc.

\section{Feature extraction and matching}

Feature extraction and matching are the backbones of most computer vision algorithms. The features are mostly extracted from a finite number of points named keypoints that are distinguished the most in the image. As a result, a lot of keypoints belong to corners or edges visible in the image which often caused by the edge or corner of an object standing next to the background. While the scene is static and the object is not moving, the features in two frames are similar and can be matched correctly. However, if the object moves with respect to the background then the appearance of the keypoint (the corner or the edge) can be changed and as a result, the feature cannot be correctly matched. 


\subsection{Motion}

\section{D triangulation}

Triangulation is a required step in most 3D computer vision applications although is not needed when using a depth camera as the 3D points are given. The process involves finding the 3D coordinate of points given matched keypoints in two frames. The keypoint that belongs to a moving object has different 3D coordinates (with respect to a world coordinate frame attached to the background) in two temporal frames which without having a motion model it cannot be retrieved.

\section{Relative pose estimation}

Incremental camera pose estimation finds the pose of a new frame based on two transformations: the pose of the previous frame with respect to the world coordinate frame, the relative transformation between the new and previous frame. To find the relative transformation of two frames different algorithms such as $2 \mathrm{D}$ to $2 \mathrm{D}$, $3 \mathrm{D}$ to $2 \mathrm{D}, 3 \mathrm{D}$ to $3 \mathrm{D}$ are proposed. In all these algorithms, the relative transformation is obtained by minimizing an error function which is the error of projection or transformation. This error is calculated as the summation of each keypoint's projection/transformation error. As keypoints are spread among static and dynamic 3D points with different transformations, minimization can fail or converge to a wrong transformation. The ideal optimization algorithm should eliminate all keypoints belong to moving objects and only use keypoints that are static which even in this case temporal information of moving objects is ignored.

\section{Loop closure}

Loop closure is the process of eliminating propagated error introduced by incremental camera pose estimation. The idea is when the camera revisits a previously seen scene, an optimization on all previous frames bundled together will reject the error propagation and refines all camera poses. The static scene assumption can easily be found in the loop closure as revisiting a scene whose objects are moving cannot be achieved if one looks at the scene with everything in it. As a result, the loop closure cannot be done for the entire scene if one wants to have temporal information of moving objects. 


\section{Chapter 4}

\section{Literature Review}

In this chapter, a review of algorithms for 3D registration and reconstruction is given. Depending on the sensor data, one can apply one of these algorithms to generate a complete 3D model of a rigid scene. The registration algorithms are widely used with depth or Lidar sensors that capture 3D frames of the scene as the sensor explores the environment and the algorithm tries to best align all partial clouds of points and merge them together to create a complete model of the scene. Therefore, the main challenge is to find the pose of frames that best merge all clouds of points. On the other hand, 3D reconstruction algorithms not only have to estimate camera poses but also need to estimate 3D points visible in each frame of the 2D image. Simultaneous Localization and Mapping (SLAM) also known as Structure from Motion (SfM) in the computer vision community, is the problem of finding the robot pose (position and orientation) while moving in an environment and building the map of this environment simultaneously. While the main focus in SLAM is on accurate estimation of the robot (camera) pose on the fly, SfM pays more attention to the structure, namely the map of the environment.

In the next sections, an overview of registration and reconstruction algorithms is given and their weaknesses are discussed specifically for dynamic scenes. 


\subsection{Registration}

\subsection{Registration}

Registration of 3D models of an object is the process of finding a rigid transformation that aligns the source cloud to the target cloud. Rigid-body registration is an important problem in computer vision, computer graphics, and robotics and has application in other problems like SLAM, 3D object retrieval, camera relocalization, object pose estimation, etc. Looking at the literature, a common solution involves two stages: a coarse global registration to estimate an initial rigid transformation that aligns two clouds relatively close to each other, and a local refinement stage that refines the initial estimate and obtains a better registration [37-39]. The first stage mostly relies on point-to-point correspondences found using a 3D descriptor and compute the initial transformation iteratively from sparse random draw subsets of correspondences using a variant of RANSAC. However, this process takes many iterations when the data is noisy or the models partially overlap. The second stage, the local refinement stage, uses the initial estimate of the transformation and refines the estimation based on dense correspondences. This stage is mostly based on iterative closest point (ICP) algorithm or its variants [40,41]. The ICP algorithm refines the initial estimate by iteratively establishing correspondences via closestpoint lookups and recomputing the transformation based on new correspondences. The initialization plays a crucial role in ICP and it often fails if the initial estimate is not close to the optimal solution. The combined two-stage registration pipeline achieves robust registration however, such registration pipeline is computationally expensive as both model fitting and the local refinement steps are iterative. The run time speed is a crucial factor in registration as they are often used as part of the SLAM algorithm to map the environment in real-time (30 fps).

\subsection{SLAM or SFM}

Simultaneous Localization and Mapping (SLAM) or Structure from Motion (SFM) are well-known topics in robotics and computer vision where the goal is to simultaneously find the pose of the robot (or camera) and create a map for the environment the robot is exploring. In this section, an overview of the SLAM algorithms and a 


\subsection{SLAM or SFM}

list of strengths and weaknesses of each approach are discussed.

Looking at the literature, different theoretical approaches have been proposed to solve the SLAM problem. These solutions were introduced for different domains from indoor to outdoor, underwater, and airborne robots. Also, different sensor data (Monocular RGB, Stereo RGB, RGBD, LiDAR, Sonar, GPS, IMU), as well as their fusion, were used for SLAM. Depending on sensor data, these algorithms can be categorized into 1) Volumetric methods where raw data (2D or 3D) are directly fed into the system; and 2) Feature-based methods where a subset of raw data are used (landmarks) after some preprocessing (feature extraction). The latter was done to make the SLAM scalable and reduce the computational complexity of the problem. From the theoretical point of view, the SLAM algorithms can be categorized into two frameworks explained below:

\subsubsection{Bayes Filter Framework}

The Bayes filter is a framework to estimate the probability distribution of the state of the system $(x)$ given the observations $(z)$ and the controls $(u)$. In other words:

$$
\begin{aligned}
& \operatorname{bel}\left(x_{t}\right)=p\left(x_{t} \mid z_{1: t}, u_{1: t}\right) \\
& =\eta p\left(z_{t} \mid x_{t}, z_{1: t-1}, u_{1: t}\right) p\left(x_{t} \mid z_{1: t-1}, u_{1: t}\right)
\end{aligned}
$$

The Bayes Filter, as the name suggests, uses Bayes rule along with some assumptions and probability rules to make the above equation in a recursive form so one could estimate the current state of the system using only the previous state of the system and current observation and control signal. This is usually done in two phases, predication, and correction (estimation) steps:

Prediction: $\overline{\operatorname{bel}}\left(x_{t}\right)=\int p\left(x_{t} \mid x_{t-1}, u_{t}\right) \operatorname{bel}\left(x_{t-1}\right) d x_{t-1}$

Correction: $\operatorname{bel}\left(x_{t}\right)=\eta p\left(z_{t} \mid x_{t}\right) \overline{\operatorname{bel}}\left(x_{t}\right)$

From these equations, one could see the prediction step is using the motion model of the robot $\left(p\left(x_{t} \mid x_{t-1}, u_{t}\right)\right)$, that is, given the previous state of the system and the control command how the robot is moved. On the other hand, the correction step is using the observation model $\left(p\left(z_{t} \mid x_{t}\right)\right)$, that is, given the state of the system, what should be the observation from the sensor data. The above equations are obtained using the following assumptions and rules: 
- given that the state of the system is known, the previous measurements and controls can be ignored (Markov assumption):

$$
p\left(z_{t} \mid x_{t}, z_{1: t-1}, u_{1: t}\right) \sim p\left(z_{t} \mid x_{t}\right)
$$

- then, using the law of total probability and Markov assumption:

$$
\begin{aligned}
& p\left(x_{t} \mid z_{1: t-1}, u_{1: t}\right)=\int_{x_{t-1}} p\left(x_{t} \mid x_{t-1}, z_{1: t-1}, u_{1: t}\right) p\left(x_{t-1} \mid z_{1: t-1}, u_{1: t}\right) d x_{t-1} \\
& =\int_{x_{t-1}} p\left(x_{t} \mid x_{t-1}, u_{t}\right) p\left(x_{t-1} \mid z_{1: t-1}, u_{1: t-1}\right) d x_{t-1} \\
& =\int_{x_{t-1}} p\left(x_{t} \mid x_{t-1}, z_{1: t-1}, u_{1: t}\right) \operatorname{bel}\left(x_{t-1}\right) d x_{t-1}
\end{aligned}
$$

In the context of SLAM, the state of the system $\left(x_{t}\right)$ is a vector containing pose of the robot and landmarks (localization and mapping). For localization alone (given the map), the state of the system $\left(x_{t}\right)$ will be reduced to the only pose of the robot.

The Base filter is a general framework for recursive state estimation without any assumptions on the underlying distribution or how to compute the integrals. To solve the SLAM problem, different algorithms have been proposed that are considered variants of Bayes filter some listed below:

\subsubsection{Parametric Filters}

\section{Kalman Filter (KF)}

Kalman filter is an implementation of Bayes filter with assumptions that the underlying distributions are Gaussian and the motion and observation models are linear which makes the Kalman filter an optimal solution for SLAM with the abovementioned assumptions. Given the linear motion and observation model as:

$$
\begin{aligned}
& x_{t}=A_{t} x_{t-1}+B_{t} u_{t}+\epsilon_{t} \\
& z_{t}=C_{t} x_{t}+\delta_{t}
\end{aligned}
$$

where $A_{t}$ is $n \times n$ matrix describing how the state of the system changes without control inputs and noise, $B_{t}$ is $n \times l$ matrix describing how the control inputs change the states of the system, and $C_{t}$ is $k \times n$ matrix representing observation model.

Using properties derived from linearity of the models and Gaussian distributions, the Bayes equations can be derived and written as follows:

$$
\bar{\mu}_{t}=A_{t} \mu_{t-1}+B_{t} u_{t}
$$




$$
\begin{aligned}
& \bar{\Sigma}_{t}=A_{t} \Sigma_{t-1} A_{t}^{T}+R_{t} \\
& K_{t}=\bar{\Sigma}_{t} C_{t}^{T}\left(C_{t} \bar{\Sigma}_{t} C_{t}^{T}+Q_{t}\right)^{-1} \\
& \mu_{t}=\bar{\mu}_{t}+K_{t}\left(z_{t}-C_{t} \bar{\mu}_{t}\right) \\
& \Sigma_{t}=\left(I-K_{t} C_{t}\right) \bar{\Sigma}_{t}
\end{aligned}
$$

where $\mu_{t}$ and $\Sigma_{t}$ are mean and covariance of the new estimation. Being a Gaussian distribution, the state of the system can be described only by the mean and covariance matrices which are incrementally estimated at each time instance.

\section{Extended Kalman Filter (EKF)}

As the linearity of motion and observation models are rarely valid in the SLAM problem, Extended Kalman Filter solves the problem by linearizing the non-linear models around the current state of the system.

$$
\begin{aligned}
& \bar{\mu}_{t}=g\left(u_{t}, \mu_{t-1}\right) \\
& \bar{\Sigma}_{t}=G_{t} \Sigma_{t-1} G_{t}^{T}+R_{t} \\
& K_{t}=\bar{\Sigma}_{t} H_{t}^{T}\left(H_{t} \bar{\Sigma}_{t} H_{t}^{T}+Q_{t}\right)^{-1} \\
& \mu_{t}=\bar{\mu}_{t}+K_{t}\left(z_{t}-h\left(\bar{\mu}_{t}\right)\right) \\
& \Sigma_{t}=\left(I-K_{t} H_{t}\right) \bar{\Sigma}_{t}
\end{aligned}
$$

where $G_{t}$ and $H_{t}$ are linearized matrices obtained by gradient of non-linear motion $g(x)$ and observation $h(x)$ models.

\section{Unscented Kalman Filter (UKF)}

While Extended Kalman Filter (EKF) solves the problem of non-linearity of motion and observation models by linearization, Unscented Kalman Filter (UKF) uses the unscented transform to estimate the required mean and covariance obtained through a non-linear function. The unscented transform uses a set of so-called sigma points drawn from the source and transforms each of the points by the non-linear function and later finds the required mean and covariance from the transformed sigma points. The main advantage of UKF is that linearization is not required which is particularly important when the underlying non-linear function is not differentiable.

Given the mean and Covariance at time $t-1$, the sigma points along with their weights can be obtained as: 
$\chi_{t-1}=\left(\mu_{t-1}, \mu_{t-1}+\gamma \sqrt{\Sigma_{t-1}}, \mu_{t-1}-\gamma \sqrt{\Sigma_{t-1}}\right)$

These points can be easily transformed using motion model to find the state of the sigma points at time $t$ :

$\bar{\chi}_{t}^{*}=g\left(u_{t}, \chi_{t-1}\right)$

The mean and covariance of the state of the system at time $t$ derived from motion model can be obtained by :

$$
\begin{aligned}
& \bar{\mu}_{t}=\sum_{t=0}^{2 n} \omega_{m}^{[i]} \chi_{t}^{*[i]} \\
& \bar{\Sigma}_{t}=\sum_{t=0}^{2 n} \omega_{c}^{[i]}\left(\bar{\chi}_{t}^{*[i]}-\bar{\mu}_{t}\right)\left(\bar{\chi}_{t}^{*[i]}-\bar{\mu}_{t}\right)^{T}+R_{t}
\end{aligned}
$$

Given the mean and covariance of the state of the system at time $t$, one could draw sigma points to be transformed by observation model:

$$
\begin{aligned}
& \bar{\chi}_{t}=\left(\bar{\mu}_{t}, \bar{\mu}_{t}+\gamma \sqrt{\bar{\Sigma}_{t}}, \bar{\mu}_{t}-\gamma \sqrt{\bar{\Sigma}_{t}}\right) \\
& \overline{\mathcal{Z}}_{t}=h\left(\bar{\chi}_{t}\right) \\
& \bar{z}_{t}=\sum_{t=0}^{2 n} \omega_{m}^{[i]} \overline{\mathcal{Z}}_{t}^{[i]} \\
& \mathcal{S}_{t}=\sum_{t=0}^{2 n} \omega_{c}^{[i]}\left(\overline{\mathcal{Z}}_{t}^{[i]}-\bar{z}_{t}\right)\left(\overline{\mathcal{Z}}_{t}^{[i]}-\bar{z}_{t}\right)^{T}+Q_{t}
\end{aligned}
$$

The fusion of the prediction and observation steps can be done similar to KF as:

$$
\begin{aligned}
& \bar{\Sigma}_{t}^{x, z}=\sum_{t=0}^{2 n} \omega_{c}^{[i]}\left(\bar{\chi}_{t}^{*[i]}-\bar{\mu}_{t}\right)\left(\overline{\mathcal{Z}}_{t}^{[i]}-\bar{z}_{t}\right)^{T} \\
& K_{t}=\bar{\Sigma}_{t}^{x, z} \mathcal{S}_{t}^{-1} \\
& \mu_{t}=\bar{\mu}_{t}+K_{t}\left(z_{t}-h\left(\bar{\mu}_{t}\right)\right) \\
& \Sigma_{t}=\left(I-K_{t} H_{t}\right) \bar{\Sigma}_{t}
\end{aligned}
$$

\section{Covariance Intersection (CI)}

One of the assumptions made for KF, EKF, and UKF was the independency of consecutive estimates or the observation and the predictions. This assumption makes the estimate to be non-conservative if the inputs are dependent. Covariance Intersection (CI) is a fusion method similar to Kalman Filter but without independency requirement of inputs. Given multiple Gaussian distributions that are not necessarily independent, the conservative fusion can be found using CI:

$$
\mathcal{G}_{1}:\left(X_{1}, P_{1}\right), \mathcal{G}_{2}:\left(X_{2}, P_{2}\right), \ldots, \mathcal{G}_{k}:\left(X_{k}, P_{k}\right) \Rightarrow\left(X_{1}, \frac{1}{\omega_{1}} P_{1}\right),\left(X_{2}, \frac{1}{\omega_{2}} P_{2}\right), \ldots,\left(X_{k}, \frac{1}{\omega_{k}} P_{k}\right)
$$

where $0 \leq \omega_{i} \leq 1$ are weights to be optimally found after applying Kalman Filter and minimizing a measure of "size" i.e. determinant or trace of the estimated 
covariance:

$$
\begin{aligned}
& P_{\text {new }}=\left(\sum_{j=1}^{k}\left(\omega_{j} P_{j}^{-1}\right)\right)^{-1} \\
& \omega_{i}=\arg \min \left(\operatorname{det}\left(P_{\text {new }}\right)\right) \text { s.t. } 0 \leq \omega_{i} \leq 1, \text { and } \sum \omega_{j}=1
\end{aligned}
$$

and the new estimate that is conservative can be found using following equation:

$$
X_{\text {new }}=\left(\sum_{i=1}^{k}\left(\omega_{i} P_{i}^{-1}\right)\right)^{-1} \sum_{i=1}^{k}\left(\omega_{i} P_{i}^{-1} X_{i}\right)
$$

\subsubsection{Non-parametric Filters}

While parametric filters rely on a fixed functional form of the posterior such as Gaussian, non-parametric filters approximate the posterior by a finite number of values i.e. samples. Histogram and particle filters are two non-parametric approaches for approximating posteriors over continuous spaces. Both approaches have little assumptions on the posterior density which makes them appropriate for complex multi-modal beliefs. The particle filter is widely used for robotics applications such as localization.

\section{Particle Filter}

The particle filters approximate the posterior by a finite number of samples namely particles. Each particle $x_{t}^{m}(1 \leq m \leq M)$ represents a hypothesis of the state of the system at time $t$. Algorithm 4.1 is a basic variant of the particle filter which the particles at time $t-1$ along with the most recent control $u_{t}$ and the observation $z_{t}$ are

used to generate hypothetical state $x_{t}^{[m]}$ for time $t$ based on the particle $x_{t-1}^{[m]}$. These hypothetical states are obtained by a so-called resampling or importance resampling (lines 8 to 11). The resampling step is done to keep all particles in regions of the high posterior probability.

\subsubsection{Graph Based Framework}

In contrast to the Bayes framework (filtering approach) that model the problem as on-line state estimation, the graph-based framework (smoothing approach) estimates the full trajectory of the robot from the full set of measurements. The graphbased SLAM as the name suggests contains a graph where nodes are robots or 


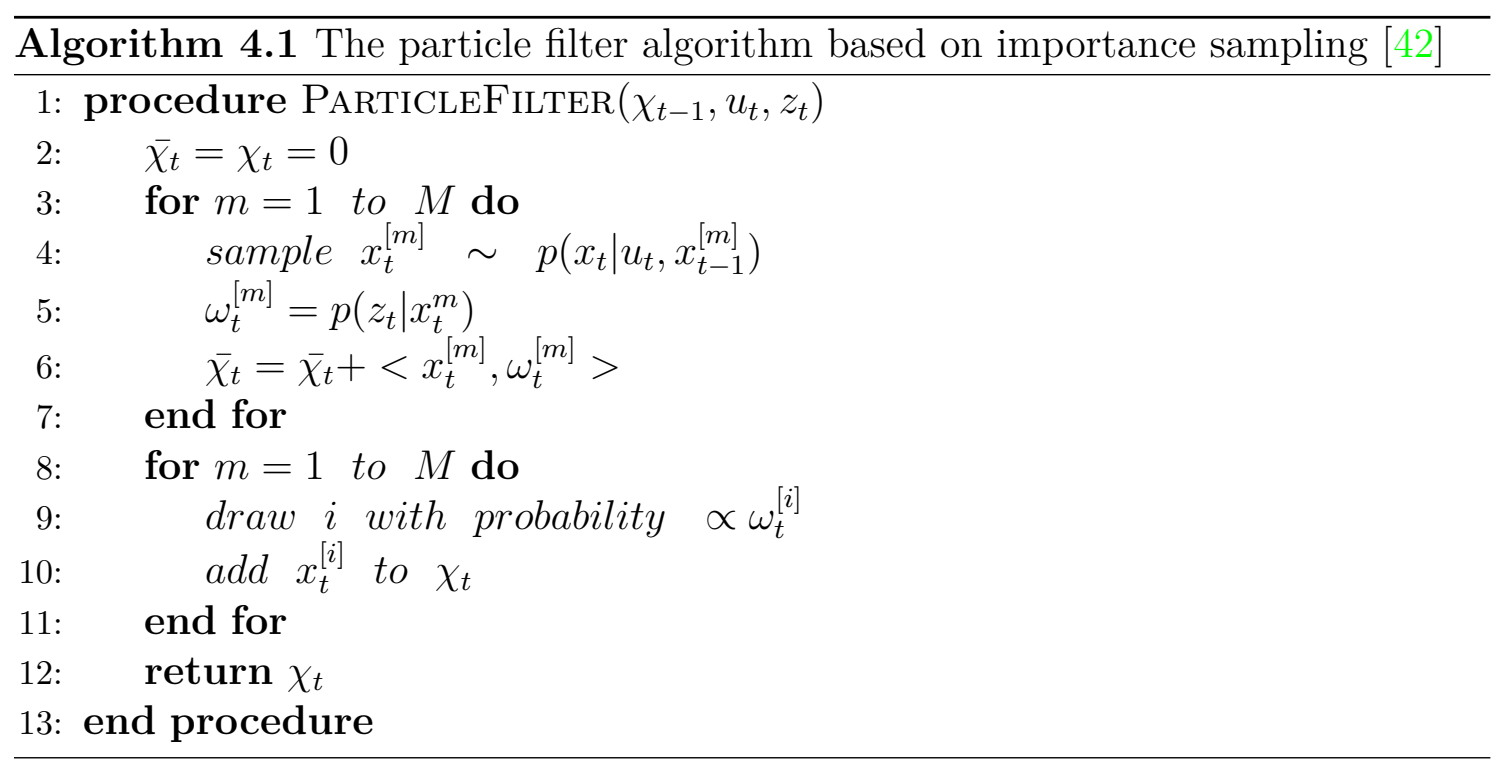

landmarks poses and edges encode constraints between nodes obtained by sensor measurements. As the sensor measurements are always affected by noise, these constraints can contradict with each other and the main problem to solve is to find a graph configuration (nodes) that maximally consistent with the measurements which involve solving a large error minimization problem [43].

Let $x=\left(x_{1}, \ldots, x_{T}\right)^{T}$ be a vector of poses where $x_{i}$ describes pose of node $i$ and can be a $2 D$ or $3 D$ transformation (i.e. Homogeneous coordinates $n D$ : $\left.\left[\begin{array}{cc}R_{n \times n} & t_{n \times 1} \\ 0 & 1\end{array}\right], n=2,3\right)$. Let $z_{i j}$ and $\Omega_{i j}$ be respectively the mean and the information matrix of the measurement between the node $i$ and $j$ and $\hat{z}_{i j}\left(x_{i}, x_{j}\right)$ be the prediction of this measurement obtained by relative transformation between the two nodes $\left(x_{i}^{-1} \times x_{j}\right)$. Let $\mathbf{e}\left(x_{i}, x_{j}, z_{i j}\right)$ be a function that computes a difference between the expected observation $\hat{z}_{i j}\left(x_{i}, x_{j}\right)$ and the real observation $z_{i j}$ :

$$
\mathbf{e}_{i j}=\mathbf{e}\left(x_{i}, x_{j}, z_{i j}\right)=z_{i j}-\hat{z}_{i j}\left(x_{i}, x_{j}\right)
$$

The goal here is to find the nodes configuration $x^{*}$ that minimizes the negative log-likelihood of all the observations between the set of pairs $(\mathbb{C})$ of indices for which an observation $z_{i j}$ exists:

$$
x^{*}=\underset{x}{\operatorname{argmin}} \sum_{(i, j) \in \mathbb{C}} \mathbf{e}_{i j}^{T} \Omega_{i j} \mathbf{e}_{i j}
$$

By formulating the SLAM problem as above, one can find the best node configuration (robot and landmark poses: $x_{i}$ ) that is consistent with all measurements. Figure 4.1 shows a pose-graph representation with different type of constraints i.e. 


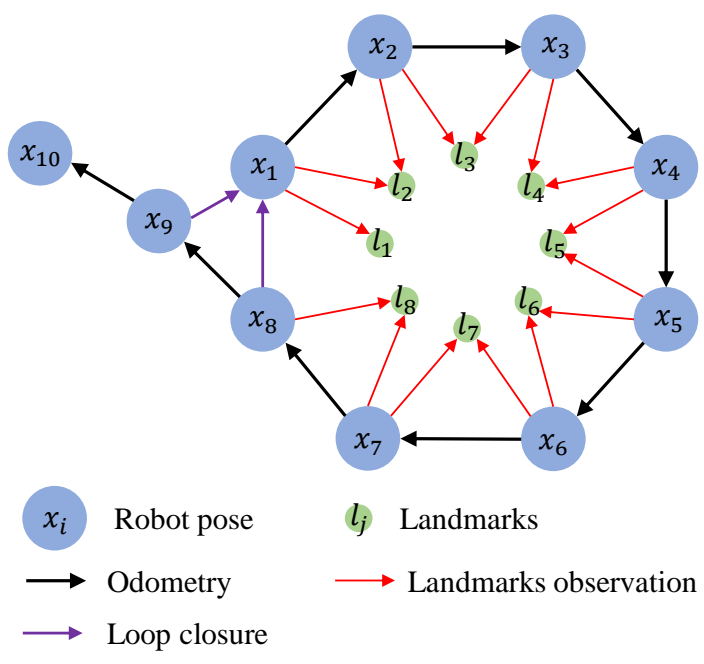

Figure 4.1: Pose graph representation of SLAM. Blue nodes are pose of robots at different time, green nodes are the position of landmarks, and arrows are different type of constraints (observations) between nodes.

odometry, landmark, loop closure.

\subsubsection{Bundle Adjustment}

Bundle adjustment (BA) is the process of refining a visual reconstruction to estimate optimal 3D structure and viewing parameters (camera extrinsic and/or intrinsic parameters) which is a key component in SfM systems. The optimization is done on an objective function that quantifies the model-fitting error where the solution is simultaneously optimal with respect to both structure and camera parameters. Mathematically, BA is a large sparse geometric parameter estimation problem where the parameters being the combination of 3D coordinates, and camera parameters. A special case of BA, motion-only bundle adjustment, aims to refine only camera parameters while 3D feature coordinates being fixed during the optimization. The motion-only BA is mostly used in depth camera where 3D feature coordinates are known. 


\section{Chapter 5}

\section{Proposed Method}

In this chapter, the proposed pipeline to process collected data using the developed platform, Vinoculer, is described in detail. The pipeline involves calibrating the data, and 3D-4D reconstruction followed by 5D registration. A non-rigid registration algorithm is also proposed to be utilized in the last stage. To visualize the processed data, a visualization tool, VisND, is developed for spatio-tempral visualization of a higher-dimensional temporal model of the canopy.

\subsection{Calibration}

Camera calibration is an essential step when dealing with images. There are different types of calibrations (thermal calibration, color calibration, geometric camera calibration, stereo camera calibration, head-eye calibration) and some were used in this

paper. For instance, thermal cameras are very sensitive and have to be calibrated regularly to produce accurate measurements. This calibration usually is conducted using black bodies, which avoid spurious radiation reflectivity, often provided by the manufacturer as well as many laboratories around the world. Below, some of the calibration procedures used in this research are described briefly:

\subsubsection{Geometric Camera Calibration}

Geometric calibration is an important step in which one finds a mathematical model relating the image pixel coordinates to their corresponding points in 3D space. This 
is commonly done using a chessboard with black and white squares that makes corner detection easy. For RGB cameras, this is relatively straight forward since a normal chessboard with black and white color can be used to calibrate the camera; however, a thermal camera is not able to detect black and white squares since the camera returns the temperature of squares. In this work, we developed a calibration pattern using a multi-material surface: i.e., thin black painted Aluminum squares on top of white paper. This combination of highly heat-absorbent and highly heatreflective materials created enough contrast for detecting corners and producing an accurate calibration - even on cloudy days. The developed pattern measured $1.5 m(H) \times 1.5 m(W)$, with 81 black aluminum squares $\left(8 \times 8 \mathrm{~cm}^{2}\right.$ each $)$. The black

aluminum and white paper squares were applied to a foam board and mounted on a sturdy, but a light wooden frame to provide support for the foam-board and two handles on the back - allowing for easy positioning of the pattern during calibration. A popular camera calibration algorithm [44] and its widely available implementation [45] were used to calibrate all three cameras with images captured at 10 different positions and orientations of the calibration pattern.

\subsubsection{Stereo Camera Calibration}

In addition to individual camera calibration which results in intrinsic and distortion camera parameters, stereo camera calibration was done for left-thermal cameras and left-right cameras. To determine the position and orientation of the cameras with respect to each other, the first calibration was executed to obtain the correct association between the measurement of the temperature and the reconstructed 3D model of plants. Figure 5.1 shows three of those images, one for each of the cameras being calibrated.

\subsubsection{Head-eye Calibration}

In mobile robotic applications, the problem of finding the camera coordinate frame with respect to the mobile robot frame is usually referred to as head-eye calibration and it can be formulated as a $A X=X B$ system of equations, where $X$ is the 


\subsection{Calibration}

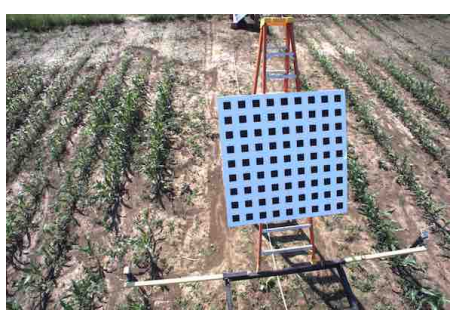

(a) Left Image

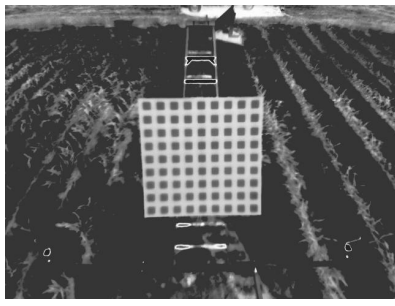

(b) Thermal Image

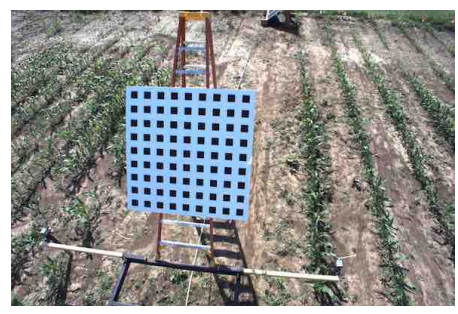

(c) Right Image

Figure 5.1: Three typical images of the aluminum + paper pattern used for calibration of RGB and IR cameras. (a) Left RGB; (b) IR Image; (c) Right RGB

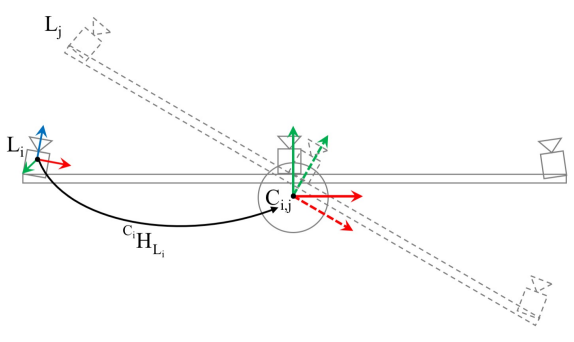

Figure 5.2: Coordinate frame of left camera with respect to turn-table (head-eye calibration)

unknown position/orientation (or pose) of the camera frame, and $A$ and $B$ are known reference frames. In this research, we used an iterative method developed by DeSouza et al. [46] to find the pose of the left camera with respect to the world coordinate frame attached to the turn table. Figure 5.2 illustrates the transformation incurred by the left camera while capturing one of the ten images obtained of the pattern at a fixed pose - i.e. ten images at different angles of the turntable such as the ones shown in Figure 5.1. For two reasons the head-eye calibration is an important step in our 4D-RGB reconstruction pipeline. First, the obtained point cloud can be referenced to a single world coordinate frame - i.e. fixed with respect to the field. This makes the phenotype features (e.g. height) and their localization in a specific plot possible. Second, all camera poses can be initially estimated with respect to the turntable's encoder, which makes the 3D reconstruction process faster (see our Constrained Bundle Adjustment below). 


\subsection{D Reconstruction Pipeline}

In order to create 5D-RGB models of the field, a sequence of processing steps is required. This pipeline started with the calibration of the trinocular cameras, as well as their head-eye calibrations. Next, the pipeline employed a 3D reconstruction algorithm, which included a constrained bundle adjustment and dense reconstruction, to combine each set of data collected during one $360^{\circ}$ revolution of the tower.

Next, it added the $4^{\text {th }}$ dimension (temperature) by back-projecting IR images onto the densely reconstructed 3D model of the field [47]. The pipeline then optimized all sets reconstructed at individual time stamp by registering the same 4D-RGB models with respect to a single, global coordinate frame defined by the first set.

\subsubsection{Pre-processing}

All camera calibration process could be performed on-site using a large calibration pattern $(1.5 \mathrm{~m} \times 1.5 \mathrm{~m})$. This calibration process consists of finding the mathematical model relating the image pixel coordinates from all cameras to their corresponding points in 3D space.

For this work, RGB and thermal cameras were calibrated using a multi-material calibration pattern that employed black-painted aluminum squares on top of white paper, on a wooden frame. This combination of heat-absorbent vs. heat-reflective, as well as high-contrast (black vs. white) materials, created enough gradient for detecting corners in both thermal and RGB images. Multi-objective optimization of stereo pairs was also carried out between the left-right (RGB) cameras and leftthermal (RGB-IR) cameras to accurately estimate the position and orientation of the cameras with respect to each other. Figure 5.1 shows three of the calibration images captured during this process.

In order to find a single world coordinate frame aligned with the observation tower, a head-eye calibration was also carried out and the pose of the left camera with respect to the center of rotation was established. This type of calibration is referred to as the $A X=X B$ problem, where $X$ is the unknown pose of the camera frame with respect to the world, and $A$ and $B$ are known reference frames. In this 


\subsection{D Reconstruction Pipeline}

paper, we used the iterative method presented in [46] to solve for the pose of the left camera, assuming a world coordinate frame at the center of the turntable.

It is important to mention here that the head-eye calibration is a very important step in the pipeline for it can affect the entire 5D reconstruction through many of the steps of the pipeline: 3D reconstruction, back-projection of thermal data onto the 3D models, and temporal cloud registration. It is also quite useful in constraining the bundle adjustment and dense reconstruction steps by reducing the complexity of the optimization from $6 \times N$ to $6+N$ unknown parameters - again, for more details, refer to [47].

\subsubsection{D Reconstruction}

In order to perform the $3 \mathrm{D}$ reconstruction of the canopy, the following steps were followed:

1. Feature extraction and three-view matching between two consecutive stereo frames: For this step, SIFT feature descriptors [48] were employed to find corresponding points between $L_{i}$ and $R_{i}$, as well as $L_{i}$ and $L_{i+1}$ images where, $L$ and $R$ represent, respectively, left and right camera frames or their images, and $i$ represents the $i^{\text {th }}$ instant (or angle of the turning table) when the images were taken. See Figure 5.3(a) for the depiction of the camera poses at various instants $i$;

2. Triangulation of stereo matches to establish 3D points: After undistorting the matched features (keypoints), a sparse set of 3D points were computed from the 2D keypoints on both left and right images. These 3D coordinates were calculated with respect to the left camera coordinate frame at instant $i$;

3. Incremental camera pose estimation: Using the Perspective from $n$ Points $(\mathrm{PnP})$ algorithm, the relative poses of the left camera frame at instant $i+1$,

i.e. $L_{i+1}$, with respect to the left camera frame $L_{i}$ were found by minimizing the error in back-projecting the 3D points at time $i$ onto the $2 \mathrm{D}$ image $L_{i+1}$. This process is iterated for $i$, creating a chain of homogenous transformation: 
${ }^{w} H_{L_{i+1}}={ }^{w} H_{L_{i}} \times{ }^{L_{i}} H_{L_{i+1}}$, where the first transformation in the chain is obtained from the head-eye calibration as the world being the center of the turn table, i.e. ${ }^{w} H_{L_{0}}={ }^{C} H_{L}$;

4. Constrained bundle adjustment to refine camera poses: As the step above iterates, the error in camera pose estimation propagates and potentially grows from $i$ to $i+1$. Therefore, the chain of camera poses needed to be refined by using a motion-only Constrained Bundle Adjustment (CBA). As mentioned earlier, this constraint - derived from the fact that the cameras rotate about a fixed $z$ axis - reduces the number of unknown parameters for optimization from $6 \times N$ to $6+N$, where $N$ is the number of left-camera poses (angles) and 6 is the number of unknowns in rotation and translation. That is, the $6+N$ parameters are: 1$)$ the $N$ relative angles $\theta_{i}^{i+1}$ of the turn table about its vertical $z$ axis, where each $\theta_{i}^{i+1}$ could be used to define the relative poses of the left camera with respect to the center of the turn table ${ }^{C} H_{L_{i}}$; and 2) the 6 rotational and translational components in ${ }^{w} H_{L_{0}}$, the refined head-eye calibration. After the refinement of the poses of all left camera frames, the poses of the right and thermal camera frames were found using the extrinsic parameters from the calibration step $\left({ }^{L} H_{R}\right.$ and ${ }^{L} H_{T}$, respectively). The CBA not only led to faster convergence, but it also reduced the residual error and hence, improving the next step, the dense reconstruction;

5. Dense 3D reconstruction using refined camera poses: Using the multi-view dense reconstruction algorithm (PMVS2) developed by Furukawa and Ponce [49] and the refined left and right camera poses from CBA, a dense 3D point cloud was created. The patches constructed by the PMVS2 algorithm were also useful in finding candidates for the thermal images from which corresponding 3D points could be observed.

\subsubsection{D Reconstruction (Temperature)}

In order to find temperature information for 3D points, the software pipeline backprojected the $3 \mathrm{D}$ points onto the thermal images from which those points were 


\subsection{D Reconstruction Pipeline}

observed. As mentioned above, the candidates for those thermal images were determined using the PMVS2 patch information. The back-projection was done using the intrinsic parameters of the thermal camera obtained during calibration and the angles $\theta_{i}^{i+1}$ above, which fully determined the transformation between the left coordinate frame at instant $i$ and the world coordinate frame, and hence also the thermal coordinate frame and the world. In other words:

$$
\begin{gathered}
{ }^{T_{i}} H_{w}={ }^{T} H_{L} \times{ }^{L_{i}} H_{w} \\
\left(\begin{array}{c}
u \\
v \\
s
\end{array}\right)=K_{T} \times{ }^{T_{i}} H_{w} \times\left(\begin{array}{c}
X \\
Y \\
Z \\
1
\end{array}\right)
\end{gathered}
$$

where ${ }^{T_{i}} H_{w}\left({ }^{L_{i}} H_{w}\right)$ is the transformation from the world coordinate frame to the $i^{\text {th }}$ thermal (left) coordinate frame; while ${ }^{T} H_{L}$ and $K_{T}$ are the extrinsic and intrinsic parameters of the thermal camera from the calibration step. Finally, to minimize the effect of potentially inaccurate 3D-to-2D back-projections, the algorithm took the median of all valid temperature values corresponding to the $3 \mathrm{D}$ points. Later, the temperature information for the point cloud was used as input for the Visualization tool (VisND).

\subsubsection{D Registration (Temporal)}

Our ultimate goal is to create a tool to allow visualization and analysis of 5D-RGB models, that is, of 4D-RGB models over time. Similarly to registering 2D images to obtain 3D models, and then 3D point clouds to obtain the 4D-RGB models, in order for us to register 4D-RGB over time, corresponding keypoints among 4D models need to be found, selected and then registered with respect to the world coordinate frame. Manual registration of $4 \mathrm{D}$ point clouds can be tedious and time consuming for large numbers of points in the cloud. Alternatively, one could use the camera poses already determined with respect to the world coordinate frame to 


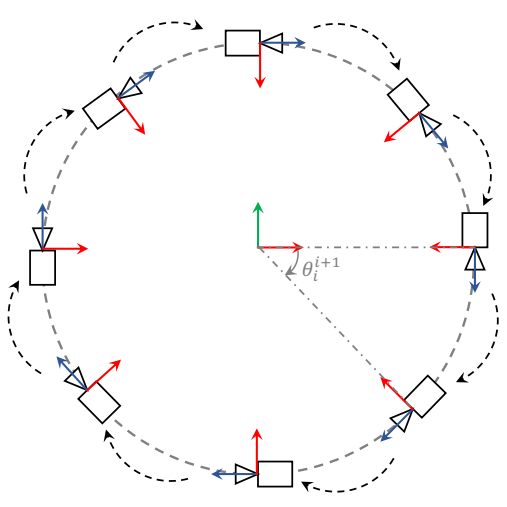

(a)

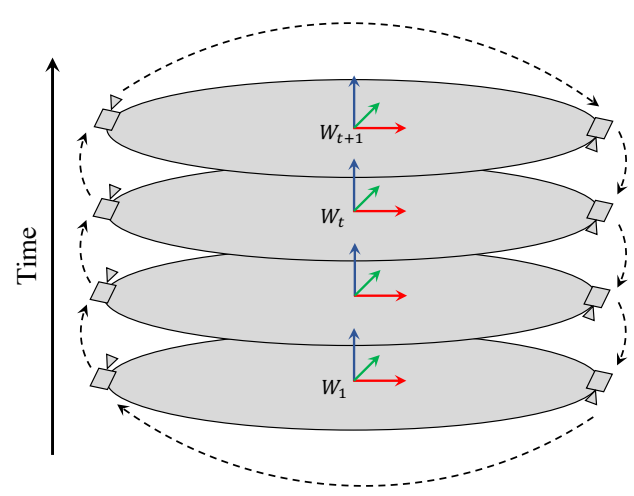

(b)

Figure 5.3: Depiction of the (a) spatial and (b) temporal constraints used for the creating of the 5D-RGB models

devise an automated registration. However, due to changes in the environment over days or even hours, finding correspondences in 4D-RGB point clouds can be very hard and error prone. Therefore, relying on incrementally updating camera poses using the relative transformations between two consecutive time frames (similar to what was done before for ${ }^{L_{i}} H_{L_{i+1}}$, but now with $i$ representing time) could lead to even greater problems in error propagation due to weak correspondences. Earlier in the spacial case, strong correspondences were possible due to the large overlaps between consecutive frames and hence a loop constraint, as in Figure 5.3(a), could be used.

Since a similar loop constraint is only possible for the temporal case if we can find strong correspondences, we limited the search for keypoints to ground pixels over periods of time no longer than 5 days (Figure 5.3(b)). Therefore, in order to find ground points, the pipeline used a color-based ground detection algorithm ( [50]) to segment out ground pixels, and then used a mask to detect keypoint features. Figures 5.4 illustrate this idea where SIFT features could be reliably matched among two temporal frames, 5 days apart, if only ground segmented pixels are used. That is, initially, in Figure 5.4(a), we observe the mismatches due to changes in the canopy, while much better results were obtained by the use of the segmentation mask in Figure 5.4(b) leading to a strong feature correspondence shown in Figure 5.4(c) after imposing that same segmentation mask.

The optimization steps for the parameters of the temporal loop were very similar 


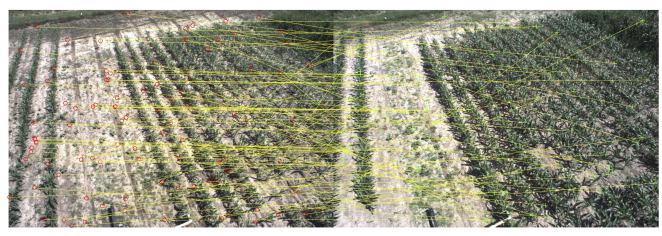

(a) Feature Matching without using ground segmentation mask

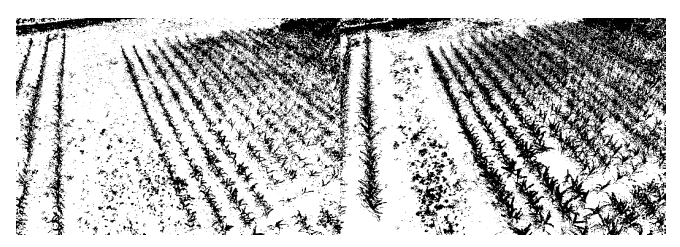

(b) Ground segmentation mask

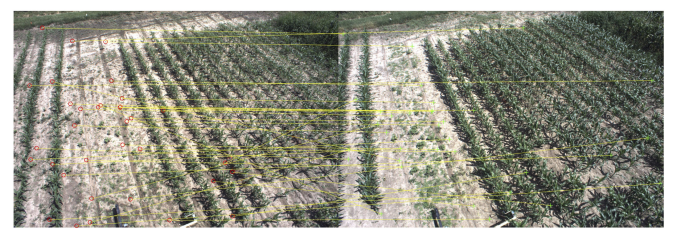

(c) Feature matching using ground segmentation mask

Figure 5.4: Temporal feature matching between images taken 5 days apart

to the ones explained in section 5.2.2, parts 3 and 4 . The differences were:

1. Before, the loop constraint (Figure 5.3(a)) was given by a chain of $N$ matrices ${ }^{L_{i}} H_{L_{i+1}}$ defined by two sets of unknowns: the $N$ times $\theta_{i}^{i+1}$ relative angles of rotations of the turn-table - which uniquely defined ${ }^{C} H_{L_{i}}$-and the 6 rotational and translational components in the head-eye calibration ${ }^{w} H_{L_{0}}$. However, for the temporal case, the loop constraint (Figure 5.3(b)) was given by $T$ matrices ${ }^{L_{t}} H_{L_{t+1}}$ defined by the unknown matrices ${ }^{W_{t}} H_{W_{t+1}}$ and the constant matrices (obtained from the spacial optimization phase) ${ }^{W_{t}} H_{L_{j}}$. Here, $t$ is the time index for the $4 \mathrm{D}$-RGB model, and $j=\{1,2\}$ is one of the two cameras empirically chosen to form the loop.

2. Instead of using a constrained bundled adjustment, the pipeline for the temporal case must run a full motion-only bundle adjustment, since now a full set of rotational and translational parameters are required to determine ${ }^{W_{t}} H_{W_{t+1}}$, leading to a total number of parameters to be optimized equal to $(6 \times(T-1))$. 


\subsection{Non-Rigid Registration}

The resulting ${ }^{{ }^{t}} H_{W_{t+1}}$ matrices were used to register the 4 D-RGB models with respect to one single coordinate frame - the first one in time. Alternatively, these transformations could be calculated by another method and provided to VisND as input.

\subsection{Non-Rigid Registration}

The last stage of the 5D reconstruction pipeline was designed to register temporal models of the canopy. As described above, by limiting the constraints to be within a 5-day period, a static scene assumption was made. That is, we used incremental temporal model rigid registration (every two consecutive $4 \mathrm{D}$ models with at most 5-days gap) and solved the error propagation problem by constructing a virtual loop where two sets of frames used to go forward and backward in time to close the loop. An alternative way is to use a non-rigid registration algorithm to align multiple deformable surfaces together. In such cases, we do not need to limit the constraints to be within a 5-day period (as long as we can find point correspondences) as the model can handle deformation as opposed to rigid transformation (rotation and translation).

\subsubsection{Non-Isometric Transformation}

As the first step towards non-rigid registration, a non-isometric registration is proposed where in addition to rotation and translation, three scale parameters in threeaxis directions are considered:

$$
H=\left[\begin{array}{cc}
S \times R & \vec{t} \\
\overrightarrow{0}^{T} & 1
\end{array}\right] \quad S=\left[\begin{array}{ccc}
s_{x} & 0 & 0 \\
0 & s_{y} & 0 \\
0 & 0 & s_{z}
\end{array}\right]
$$

where $H$ is non-rigid transformation and $S$ is the diagonal scale matrix:

Considering two point clouds: source, $q$, and target $p$, the goal is to find a nonisometric transformation $\mathrm{H}$ that aligns $p$ to $q$. The proposed method is a featurebased approach where correspondences are used to optimize the objective function. The correspondences between 3D points can be found from the corresponding 2D 
image or by computing a 3D feature descriptor like FPFH.

Let $\kappa=\{(p, q)\}$ be the set of correspondences. The objective is to optimize nonisometric transformation $H$ such that euclidian distances between corresponding points are minimized. However, due to the noise some of the correspondences might be mismatched which in such case the optimization uses wrong information and as a result converges to a local minimum. To avoid this, we use scaled Geman-McClure estimator as the robust penalty function $\rho($.) to penalize spurious constraints (large euclidian distance) and define our objective as follows:

$$
F(X)=\sum_{(p, q) \in \kappa} \rho(\|p-H q\|) \quad \text { where } \rho(x)=\frac{\mu x^{2}}{\mu+x^{2}}
$$

Figure 5.5(a) shows an interesting property of Geman-McClure robust estimator. When $\mu$ is chosen to be large, the penalty function has a small slope for a large value of $x$ (residual). On the other hand for a small value of $\mu$ the function returns sub-linear growth and rapid flattening as the $x$ (residual) grows. This makes the cost function to automatically prune the outliers (mismatches). That is starting from a large value of $\mu$ the objective function has a small gradient for all correspondences which makes two point clouds to get closer. If we reduce the value of $\mu$ as we iterate, the objective function gives a smaller gradient for bad correspondences (larger residuals) and automatically removes them from the optimization. Also, looking at the objective function (Figure 5.5(b)) one can see that decreasing the value of $\mu$ makes the object function become non-convex however the local minimums can be avoided if we are close enough to the optimum solution.

To solve this optimization problem, we use a Damped Method described next.

\subsubsection{Trust Region and Damped Methods}

Let $L$ be the second order Tylor expansion of $F$ that approximates behavior of $F$ in the neighborhood of the current iterate $x$ :

$$
F(x+h) \simeq L(h) \equiv F(x)+h^{T} g+\frac{1}{2} h^{T} H h
$$

where $g \in \mathbb{R}^{n}$ is the gradient of $F$ and $H \in \mathbb{R}^{n \times n}$ is the symmetric hessian matrix.

This approximation is a good only when $h$ is sufficiently small. Here we present two methods to determine the step $h$ such that it is a descent direction and the model $L$ stays a good approximation of $F$ around $x$. In the trust region method, we 


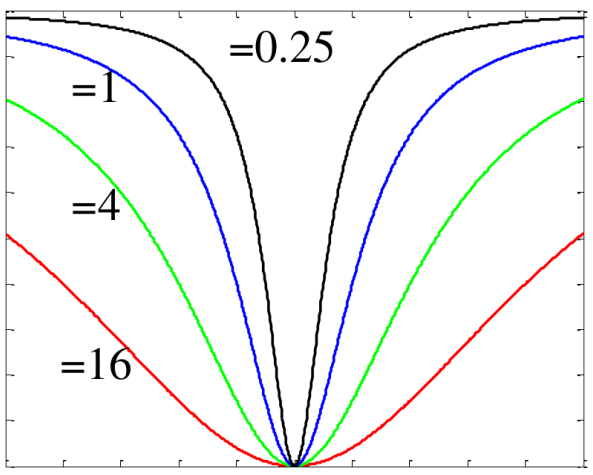

(a)

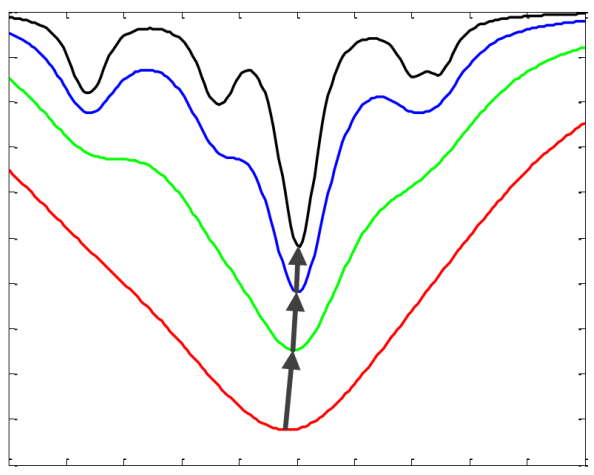

(b)

Figure 5.5: Effect of robot penalty function (a) Geman-McClure penalty function for different value of $\mu$,(b) The object function with Geman-McClure robust estimator as the penalty function [1].

assume a boundary with a size of $\triangle$ around $x$ such that the model is sufficiently accurate, and find the step $h$ as following minimization:

$$
h=h_{t r} \equiv \underset{\|h\| \leq \triangle}{\operatorname{argmin}} L(h)
$$

In the damped method, the step is determined as follows:

$$
h=h_{d} \equiv \underset{h}{\operatorname{argmin}}\left\{L(h)+\frac{1}{2} \lambda h^{T} h\right\}
$$

where the damping parameter $\lambda \geq 0$. The regularization term $\frac{1}{2} \lambda h^{T} h$ is added to penalize large steps.

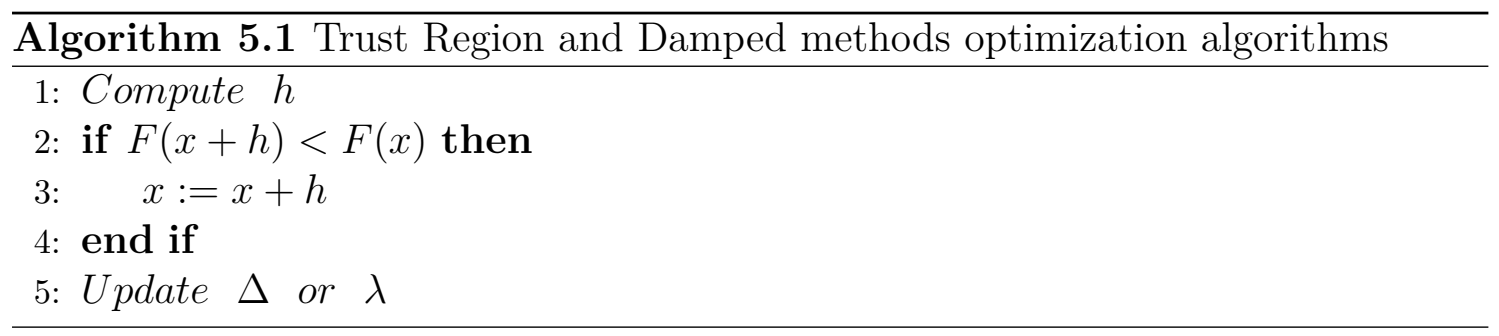

\subsection{VisND: a High Dimensional Spatio-Temporal Visualization Tool}

VisND is a tool that enables the user to visualize spatio-temporal models with additional dimensions e.g. temperature. Currently, the inputs for the software are frames of 3D point cloud, frames of temperature information, and optional transformation of frames in a world coordinate frame. VisND allows the user to play sequences of 


\subsection{VisND: a High Dimensional Spatio-Temporal Visualization Tool}

4D-RGB models as a video while providing tools for manipulating the same models; rendering different dimensions (currently color texture and temperature); measuring height and temperature of plants; and exporting that same metadata. Figure 5.6 shows an snapshot of the VisND graphical user interface (GUI). VisND was written in $\mathrm{C}++$ using Qt, PCL, and OpenCV libraries for the Linux Operating System. However, it can be easily ported to Windows or Mac OS since all libraries are cross-platform.

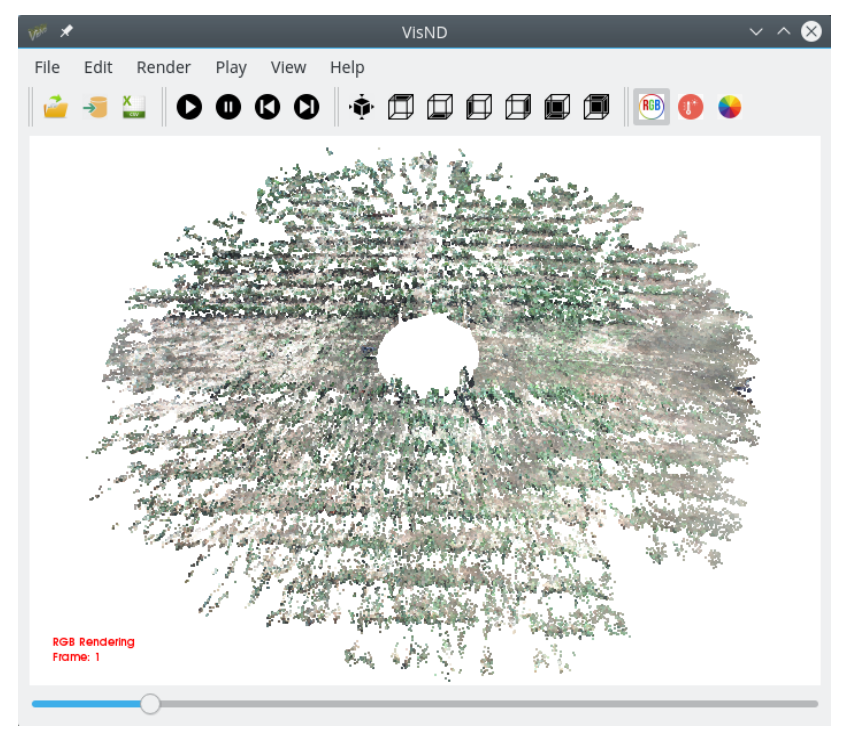

Figure 5.6: VisND Graphical User Interface (GUI)

The main features of VisND are as follows:

- Data model input: The user can import all 4D-RGB models together or add them individually. Each 4D model is composed of a 3D point cloud file (in .ply or .pcd formats), and a text file containing information on the higher dimension data - currently, temperature, but in the future other hyper-spectral data;

- 3D manipulation: Like any 3D software viewer, VisND provides tools to render the models from different viewing angles, zooming in and out, etc. It can also crop the model by setting planar thresholds. The planes are defined by clicking on any set of 3 arbitrary points and then changing its position and orientation (Figure 5.7a). This tool is useful to analyze plants and soils separately (Figure 5.7b); 


\subsection{VisND: a High Dimensional Spatio-Temporal Visualization Tool}

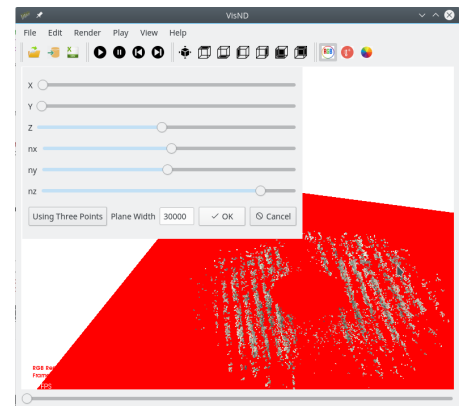

(a)

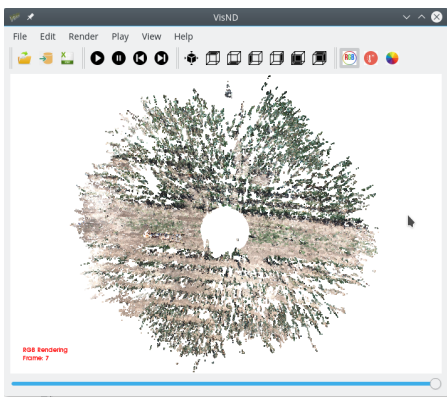

(c)

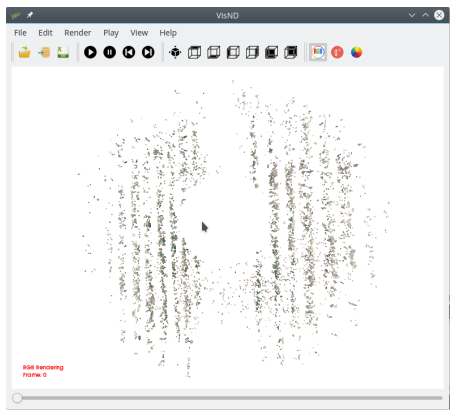

(b)

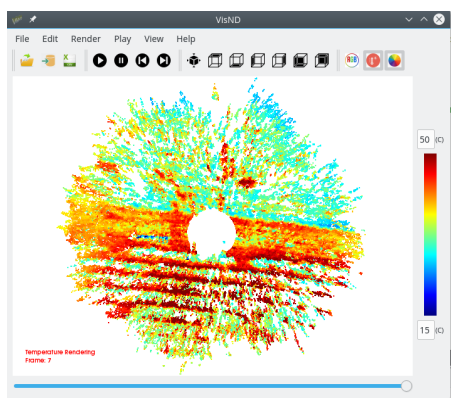

(d)

Figure 5.7: Snapshots of VisND demonstrating its current feature: (a) Manual ground detection (b) Plant segmentation by removing ground plane (c) Appearance rendering (d) Temperature rendering using Jet color mapping

- Selective model resolution: Optionally, the software can be configured to reduce the density of the point clouds. This parameter uses a voxel grid filter to reduce the cloud resolution. This can improve the rendering of large datasets with multiple models each carrying a large cloud of points;

- Animation of sequences of 4D-RGB models: This enables the user to follow temporal changes of individual plants or the entire crop. Currently, these animations can be either in $3 D$ structure $+R G B$ (Figure $5.7 \mathrm{c}$ ) or $3 D$ structure + temperature (Figure 5.7d);

- Choice of color mapping: Different color maps can be used for the visualization of higher dimensional data (again, currently temperature, but in future hyper-spectral data). This allows, for example, the user to scale the display of temperatures according to the gradients in specific areas of the model. The current options of color maps are Autumn, Bone, Jet, Winter, Rainbow, Ocean, Summer, Spring, Cool, HSV, Pink, and Hot (Figure 5.7d); 


\subsection{VisND: a High Dimensional Spatio-Temporal Visualization Tool}

- Manual registration of 4D-RGB clouds: This feature allows the user to manually click on 3D points and establish their correspondences among different 4D-RGB models. This manual selection can also be used to skip the temporal registration step of the pipeline as one can provide previously defined transformations as input;

- Extraction of phenotypes: Heights and temperatures of plants, leaves, etc. can be extracted by clicking on various points of the model. In the future, other phenotypes will be automatically extracted, such as LAI, leaf area, leaf angle, etc... These phenotypes can be exported into .csv files for future use; 


\section{Chapter 6}

\section{Experiments and Results}

In this chapter, results for the proposed spatio-temporal reconstruction pipeline as well as the non-isometric, and non-rigid registration algorithms are presented. We use the data collected by Vinoculer for the 5D reconstruction pipeline, a synthetic range datasets as well as an in-house real dataset for non-isometric registration. The synthetic data is used to enable detailed evaluation of the proposed method.

\subsection{Datasets}

\subsubsection{Vinoculer (Temporal Stereo RGB-Thermal Images)}

Various experiments and data collections have been performed at the University of Missouri Bradford Research Center. Here, we will focus on experiments conducted in one of the fields where maize and sorghum were planted in rows with either 30, 45, and 60 ", i.e. 76,114 , and $152 \mathrm{~cm}$ spacings between rows. Figure 6.1 shows the spatial configuration of the rows and plant species distribution, as well as the location of the Vinoculer in the center of the field, and two illustrations of the Vinobot as if moving between two rows. Each small vertical box in the Figure represents 10 plants, usually selected for destructive sampling - i.e. for manual phenotyping and hence, the source of ground truth data. The filled boxes indicate the plants actually used in the experiments in this paper. The red boxes mark the areas with maize and blue boxes those with sorghum. Ground truth data were collected from all row 


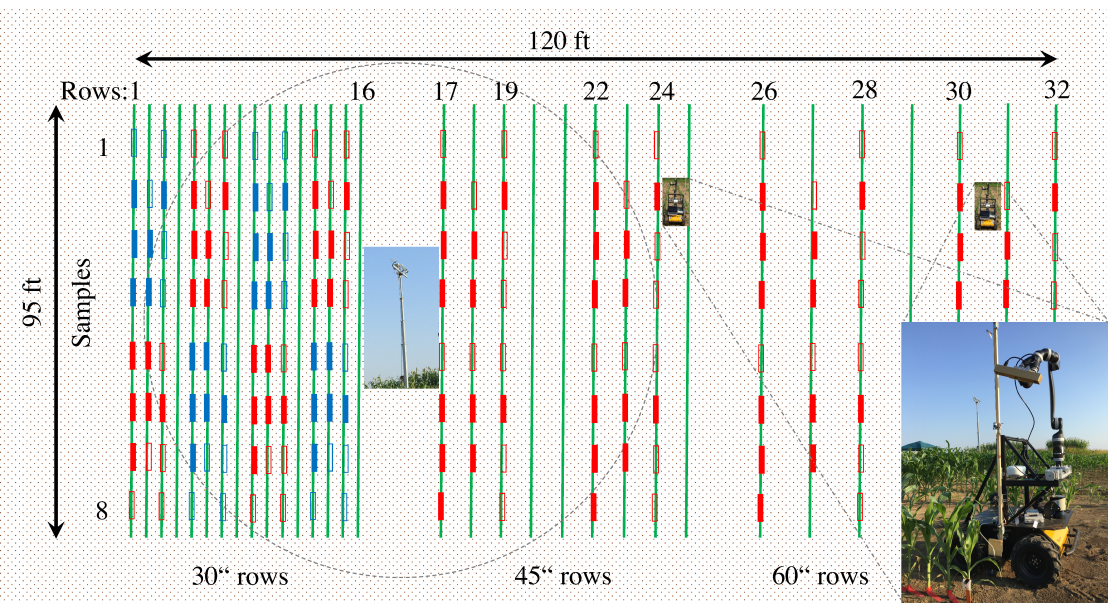

Figure 6.1: Field configuration at the MU Bradford Research Center. Small vertical boxes represent 10 plants, with sorghum marked by blue and corn marked by red boxes. Full boxes indicated plants selected for destructive sampling.

Table 6.1: Sampling method performed per indicated type of plots

\begin{tabular}{|c|c|c|c|}
\hline Plot Type vs. Sampling Method & Manually & Vinoculer & Vinobot \\
\hline \hline $30 "(76 \mathrm{~cm})$ Rows & $\sqrt{ }$ & $\sqrt{ }$ & \\
\hline $45 "(114 \mathrm{~cm})$ Rows & $\sqrt{ }$ & $\sqrt{ }$ & $\sqrt{ }$ \\
\hline $60 "(152 \mathrm{~cm})$ Rows & $\sqrt{ }$ & & $\sqrt{ }$ \\
\hline
\end{tabular}

spacings and related to Vinoculer-based data obtained for the 76 and $114 \mathrm{~cm}$ row spacings, and to Vinobot-based data obtained for the 114 and $152 \mathrm{~cm}$ row spacings. This sampling method is summarized in Table 6.1.

\subsubsection{Synthetic range data}

We used a combination of three well-known models introduced in [1]: the AIM@SHAPE repository (Bimba, Dancing Children, and Chinese Dragon), the Berkely Angel dataset [51], and the Stanford Bunny. The dataset consists of three sets (different noise level) of 25 synthesized models (5 pairs of partially overlapping range images for each type: Bimba, Dancing Children, and Chinese Dragon, Angel, and Bunny). Three different noise levels, defined by setting the standard deviation of the Gaussian distribution to $\sigma=0$ (no noise), $\sigma=0.0025$, and $\sigma=0.005$ (the unit of $\sigma$ is the diameter of the surface) are considered to better analyze the performance of the registration in presence of noise. The number of points in each range image 


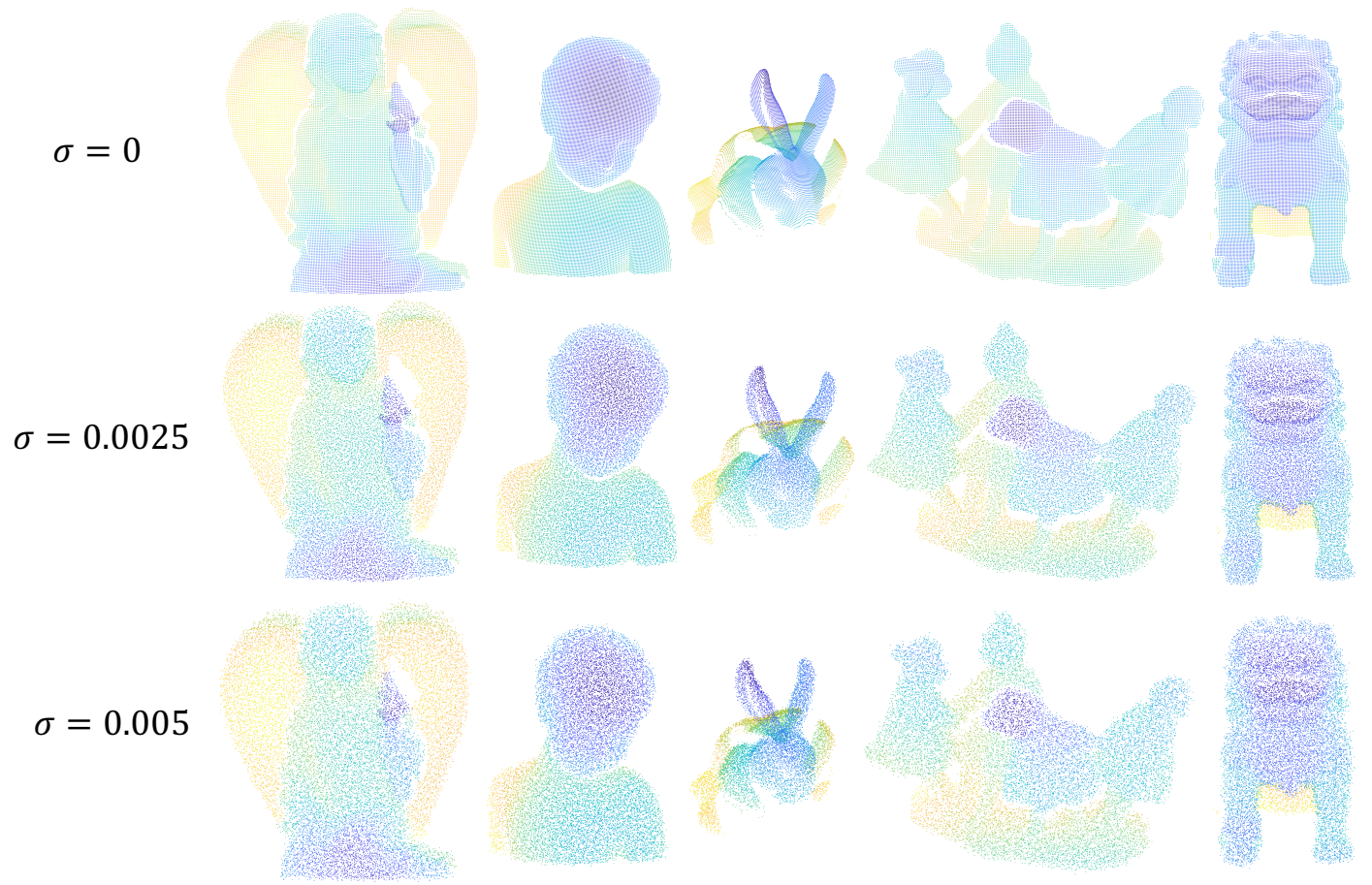

Figure 6.2: Synthetic range data

varies between $8 K$ to $19 K$ and the overlapping ratio varies between $47 \%$ and $90 \%$. Figure 6.2 shows different types of models (Bimba, Dancing Children, and Chinese Dragon, Angel, and Bunny) the data synthesized from.

\subsubsection{African violet spatio-temporal dataset}

In this work, a real-world dataset of a growing African violet plant captured with the developed $4 \mathrm{D}$ scanner is provided. The $4 \mathrm{D}$ scanner is equipped with a stereo-rig camera (top and bottom cameras), a turntable, two Raspberry Pi, and an LED lamp to help the growth of the plant, as depicted in Figure 6.3(left). The plant is placed on a turntable that rotates at specific time to allow the $4 \mathrm{D}$ scanner to capture stereo images over $360^{\circ}$ view of the plant. This data collection process was completely automated throughout the collection period. In fact, over a period of ten days, 197 sets of stereo images showing the blooming of the plant flowers were recorded and used to create dense 3D point clouds. A sample of stereo images (top and bottom images) is shown in Figure 6.3(right). 


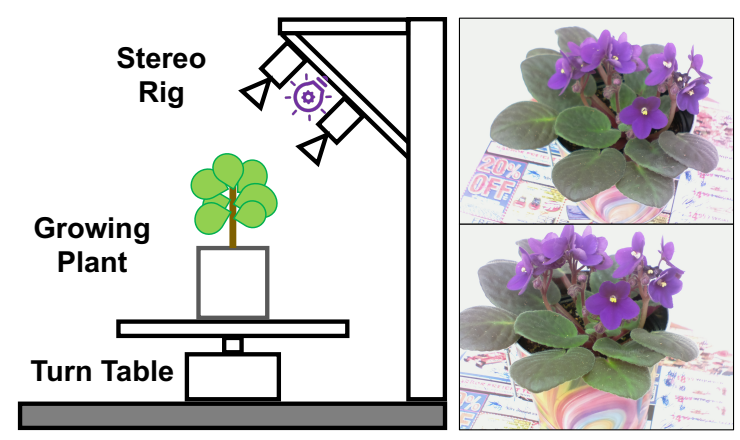

Figure 6.3: (Left) Schematic representation of the 4D Scanner equipped with a stereo-rig camera, a turn table, two raspberry pi, and a LED lamp. It can collect temporal $360^{\circ}$ view of the object. (Right) A sample stereo images: top, and bottom images captured by $4 \mathrm{D}$ Scanner

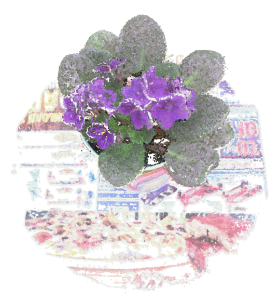

Day 1

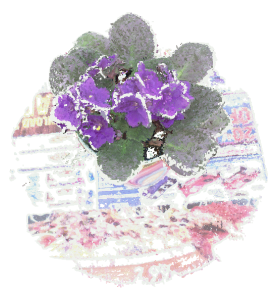

Day 4

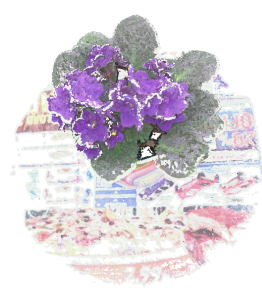

Day 7

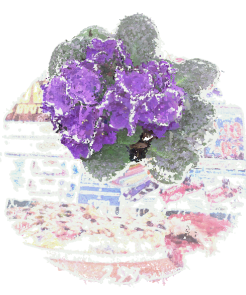

Day 10

Figure 6.4: A time-series of 3D point clouds of the same African violet plant captured during the blooming of its flowers processed using COLMAP $[2,3]$. The proposed registration algorithm is able to capture its anisotropic growth, development and deformations over a 10-days period of time. For different days, the presented clouds are obtained from raw 2D stereo images taken exactly at the same time 01:00 AM.

The dataset ${ }^{1}$, including both raw images and processed point clouds, is made publicly available for researchers in the field. Figure 6.4 illustrates examples of spatio-temporal models of African violet dataset in 4 different days.

\subsection{D Reconstruction Pipeline}

\subsubsection{Calibration}

As mentioned earlier, the Vinoculer observes the field, collecting both stereo images (for 3D reconstruction) and IR images (for inspection of leaf and soil temperatures).

\footnotetext{
${ }^{1}$ vigir.missouri.edu
} 


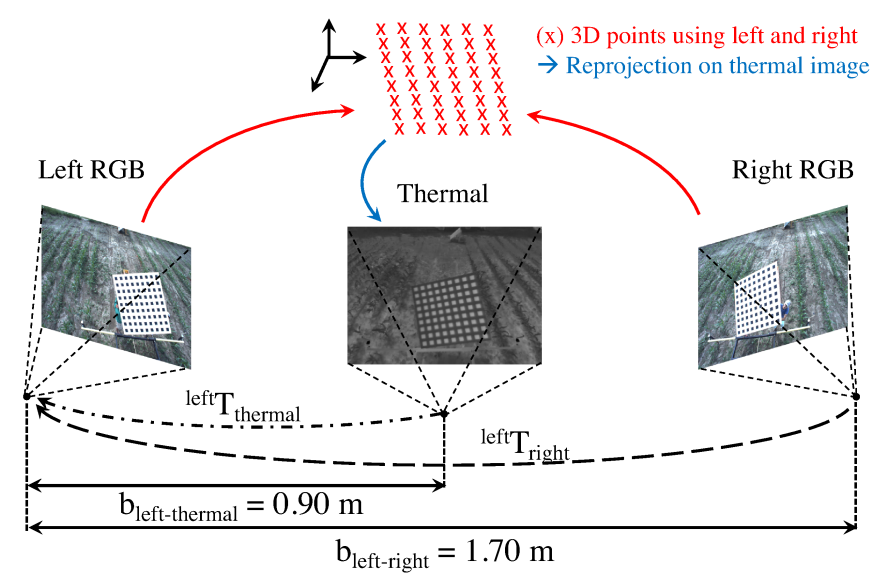

Figure 6.5: Stereo RGB-Thermal camera configuration

To register the stereo images with the IR image (i.e., to have the correct association between the measurement of the temperature and the reconstructed 3D model of a plant) a calibration procedure is required. Most calibration algorithms developed to date rely on black-and-white chessboards and finding the corners of its squares to derive a calibration matrix relating world and pixel coordinates. However, such corners on regular black and white chessboards cannot be reliably perceived by IR cameras. Thus, we developed a calibration pattern using a multi-material surface: i.e., thin black painted Aluminum squares on top of white paper. This combination of highly heat-absorbent and highly heat-reflective materials created enough contrast for detecting corners and producing an accurate calibration even on cloudy days.

From the calibration process we obtain a set of camera parameters: (1) the intrinsic parameters $K_{\text {left }}, K_{\text {right }}, K_{\text {thermal }} ;(2)$ the lens distortion $d_{\text {left }}, d_{\text {right }}, d_{\text {thermal }}$; and (3) the extrinsic parameters ${ }^{l e f t} T_{\text {right }}$, ${ }^{\text {left }} T_{\text {thermal }}$, which represents the position and orientation of the right and the thermal cameras with respect to the left, or reference camera. Figure 6.5 illustrates the spatial relationship between the cameras, with the corresponding extrinsic parameters and calibrated baselines indicated.

To validate the calibration, the calibration pattern was repositioned and 5 additional sets of images were obtained for testing. The validation was conducted using the following procedure: (1) 3D reconstruction of the corners using left and right RGB images; (2) re-projection of the $3 \mathrm{D}$ coordinates of the corners onto the thermal image; and (3) comparison between the re-projected 3D corners and the 


\subsection{D Reconstruction Pipeline}
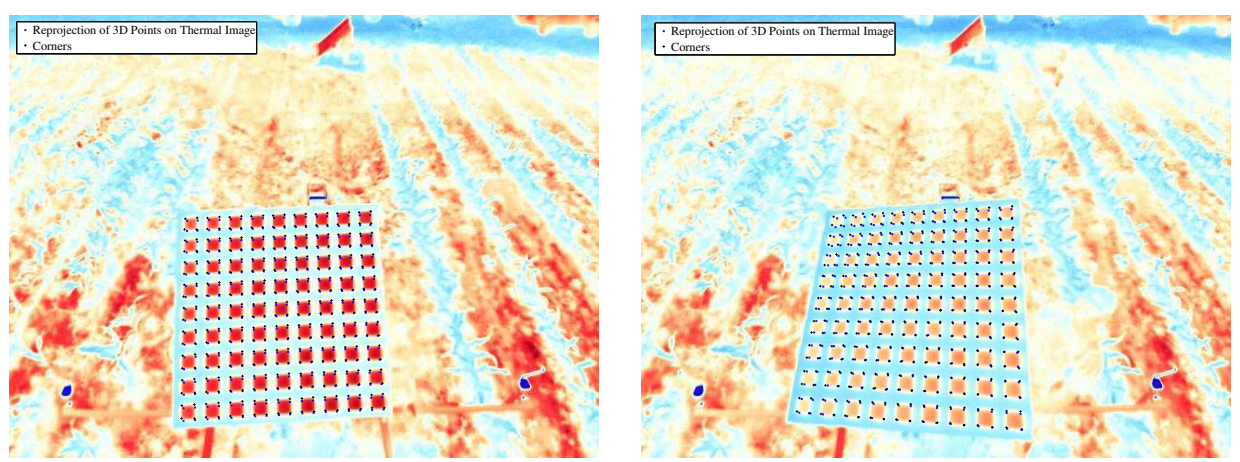

Figure 6.6: Re-projections of the 3D coordinates of the corners obtained from leftright stereo reconstruction onto the thermal images. The " $x$ " and "+" indicate the re-projections and extracted corners from the thermal image, respectively. The images were not used in calibration process.

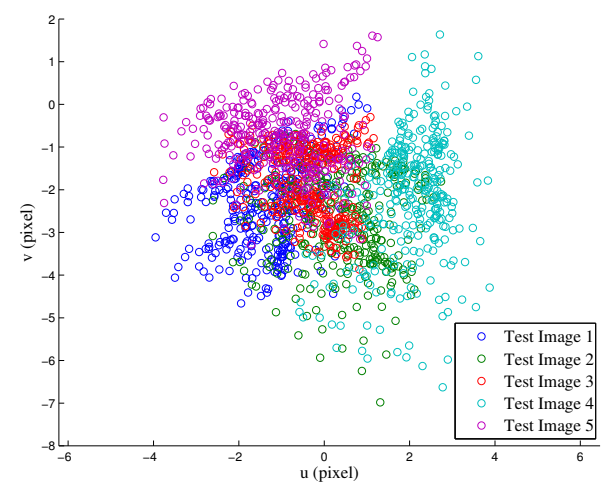

Figure 6.7: Re-projection error of 3D points found by triangulation of corners on left and right $\mathrm{RGB}$ images

extracted corners from thermal images. The rationale for this procedure is to encompass all possible sources of error, i.e., from calibration itself, 3D reconstruction, re-projection, and even corner extraction (all of which originated from the combination of right and left cameras, as well as thermal and left (reference) cameras). Both qualitative and quantitative results are reported here in Figures 6.6 and 6.7, respectively. From the latter, we calculated an RMS error of 2.5 pixels.

\subsubsection{D Reconstruction}

As detailed in Chapter 5, the 3D reconstruction is obtained by estimating $6+N$ parameters where $N$ is the number of stereo pairs collected in one turn through a constrained bundle adjustment. Based on Vinoculer stereo images, 3D models of the entire field were created to estimate the height of the canopy from the average 


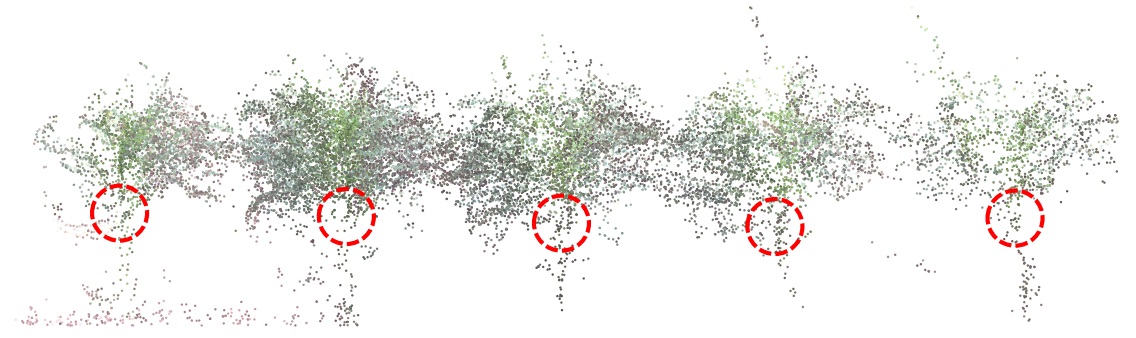

Figure 6.8: Configuration of selected points as collar of top most leaf on 3D model created by Vinoculer images

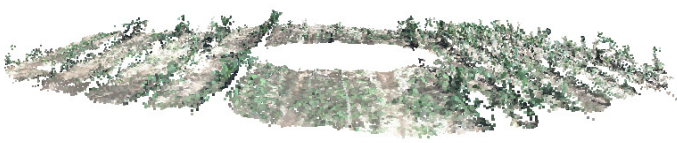

(a) $23 \mathrm{DAP}$

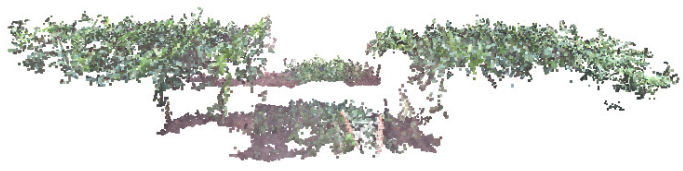

(c) $39 \mathrm{DAP}$

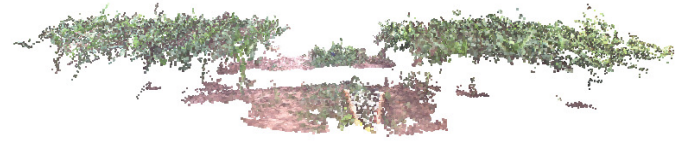

(b) 36 DAP

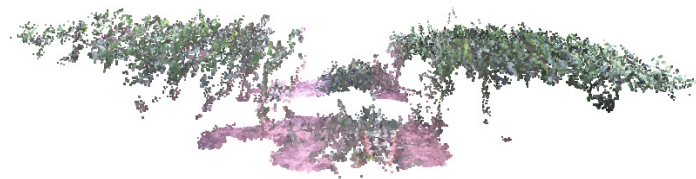

(d) 45 DAP

Figure 6.9: 3D Reconstruction of entire field (side view) using stereo $R G B$ images collected by the Vinoculer

heights of plants within a plot. Figure 6.9 depicts some of the generated 3D models (side view of the field), for different times during the growing season. From these models, the growth rate could also be calculated, and biotic and abiotic stresses could hence be investigated.

Using 3D models of the canopy, we extracted two features: height (measured by the height of the uppermost leaf collar) and Leaf Area Index (LAI). To validate our system, the averages of the heights of plants from many plots over multiple days were computed using the 3D models and compared to the ground truth data. Figure 6.10(a) shows the relationship between these measurements and their associated errors. The mean square error was calculated to be $2.36 \mathrm{~cm}$. The heights were measured by averaging, among the plants in the same plot, the distances from the collar of the top leaf to the ground (see Figure 6.8).

Leaf area index was estimated from Vinoculer images using an equivalent to airborne canopy photography by defining a new estimate of gap fraction for 3D im- 


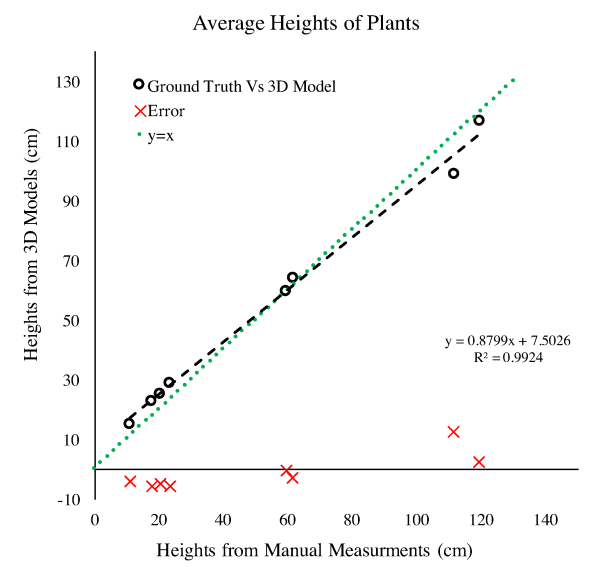

(a)

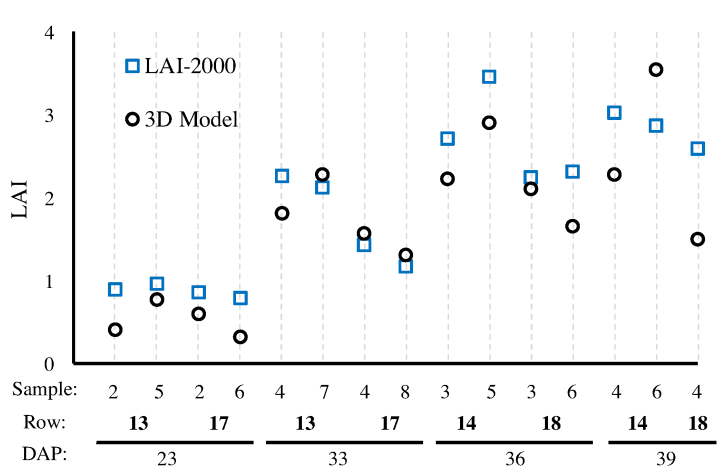

(b)

Figure 6.10: Evaluation of phenotyping features: (a) height, and (b) Leaf Area Index (LAI) extracted from 3D models

ages. That is, gap fraction is usually calculated as the ratio between the number of background pixels and the total number of pixels within the region of interest (ROI) of the image taken vertically downwards. To find background pixels, a segmentation algorithm is employed to distinguish background (soil) and foreground pixels (leaves). LAI is then commonly estimated from vertical gap fraction as [52]:

$$
L A I=-2 \ln \left(P_{0}\right)
$$

where $P_{0}$ is the vertical gap fraction.

It is important to mention here that this approach to LAI estimation is very error-prone, especially when weeds are present. So, in our system, LAI was estimated by first thresholding plants from the 3D model of the field. That is, instead of using a simple foreground and background segmentation based on color, our algorithm also took into account the heights of the point clouds. Since the presence of weeds resulted in a distinct height distribution, our height thresholding approach drastically and more accurately improved the segmentation. Once the foreground (real crop) was segmented from the background (soil), a 3D to 2D orthogonal reprojection was applied to the point clouds to create simulated top-down views of the canopy. Finally, from the knowledge of the ROIs, vertical gap fractions were calculated using the traditional method discussed above.

Figure 6.10(b) presents the LAI estimation results for different plots in the field, obtained during the growing season and compared against the manual measurements 


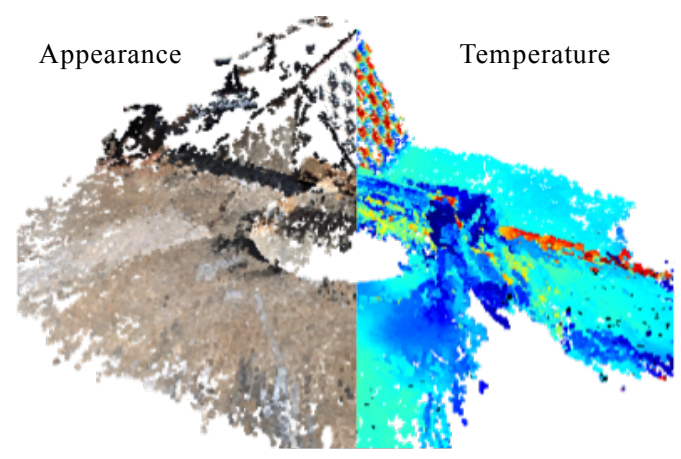

(a)

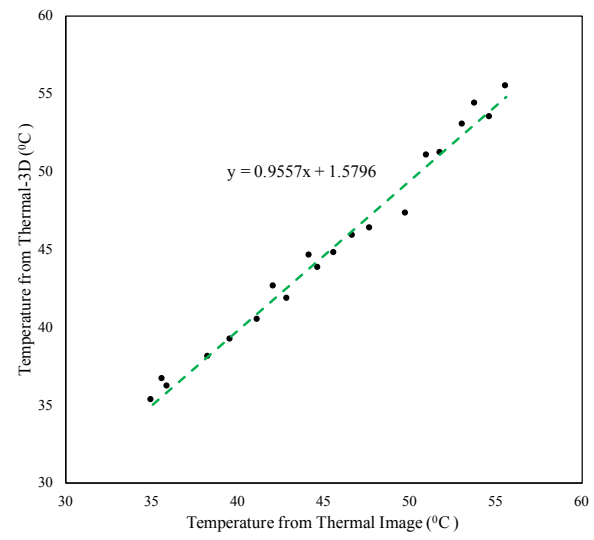

(b)

Figure 6.11: (a) RGB(left) - Thermal (right) 3D model used for quantitative evaluation of thermal 3D projection. (b) Temperature of distinct points (in RGB and Thermal images) visible in point cloud and thermal image are compared.

using LAI-2000 (ground truth). The horizontal axes shows the number of samples collected per row; the row number according to Figure 6.1; and DAP.

\subsubsection{Validation of Thermal to 3D projection}

To validate $4 \mathrm{D}-\mathrm{RGB}$ reconstruction, quantitative and qualitative analyses were conducted. In the quantitative evaluation shown in Figure 6.11(b), the temperature of distinct points (visible both in RGB and Thermal images with two adjacent materials) are measured from the point cloud and thermal image. All points were manually selected and the temperature from the point cloud and thermal images are compared to show spatial consistency in projecting temperature information into the point cloud. This evaluation is done to ensure that the projection of thermal information into 3D point cloud has no offset or drift. Again, this can be more clearly tested at the edge between hot and cool materials as in the black and white squares in the calibration pattern, also depicted in Figure 6.11(a).

In terms of qualitative results, Figure 6.12(a) shows an analysis of the thermal projections where the RGB and temperature of plants in the field are both represented. As it can be seen through the rows of plants in the RGB (top) and Thermal (bottom) parts of the figure, the projection of the thermal signature on the point cloud is aligned with the true location of their $3 \mathrm{D}$ points. That is, rows of plants 


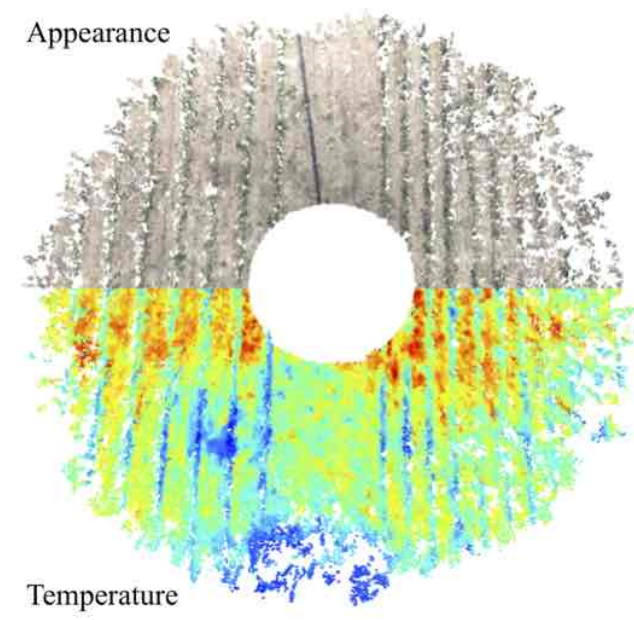

(a)

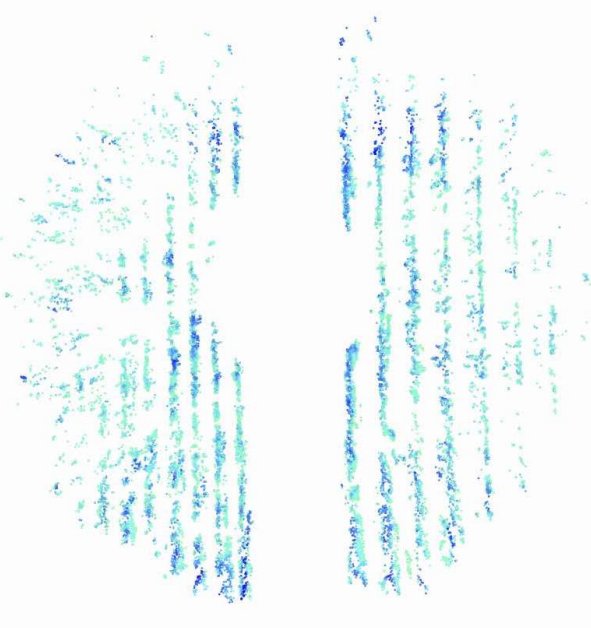

(b)

Figure 6.12: (a) RGB (top) Thermal (bottom) 4D reconstructed point cloud of the entire field at 19 DAP (days after planting), (b) Canopy temperature by ground subtraction

(easily visible in both RGB and Thermal) are perfectly aligned with each other without any offset.

\subsubsection{D-RGB Point Cloud of the Canopy}

One of the main advantages of our developed platform (Vinoculer) is its ability to monitor plants 24/7 and throughout the growing season. Also, the fusion of thermal, RGB, and 3D point cloud opens the door to visualization, inspection and analysis through the extraction of important phenotypes that can correlate plant growth rate with the genotype and environmental conditions like temperature. Having the geometric information along with the temperature can also help to maintain appropriate conditions for plants, for instance by irrigation of stressed areas of the field. Finally, visualization and analysis of 4D-RGB point cloud throughout the season allow us to better understand the long-term behavior of different genotypes. 


\subsection{D Reconstruction Pipeline}

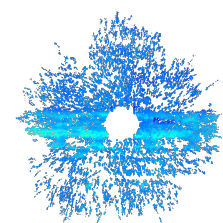

08:30

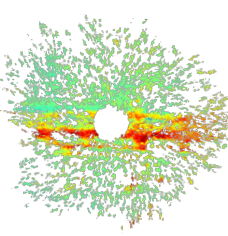

$11: 30$

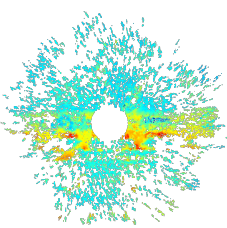

15:00

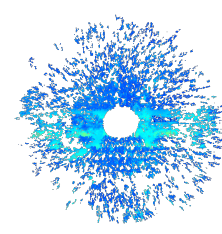

$17: 30$

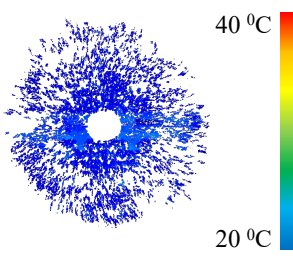

19:30

Time

Figure 6.14: Snapshot of Thermal 3D models of the field at different times at 38 DAP
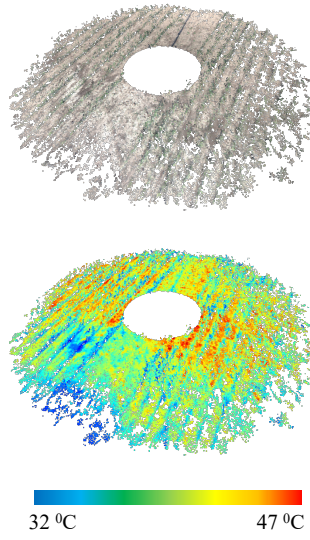

DAP 19
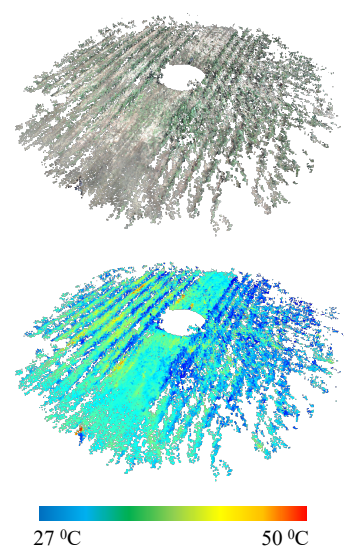

DAP 24
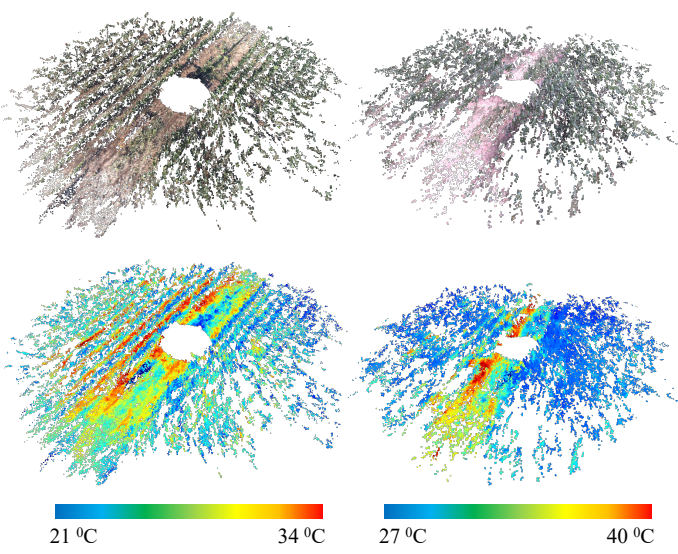

DAP 29

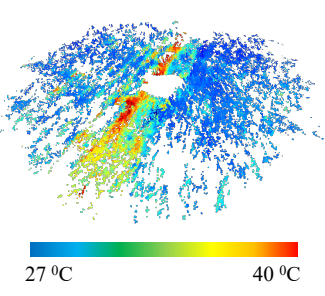

DAP 38

Figure 6.13: Snapshot of 4D-RGB models of the field throughout the season at four DAPs

In that sense, Figure 6.13 shows 3D models of the field with appearance and temperature information at four different DAPs (days after planting) 19, 24, 29, and 38. Similarly, Figure 6.14 shows the variation of temperature in the field throughout the day. For early developmental stages, when the canopy is not closed yet, both canopy and soil temperatures, two important measurements for plant biologists, can be extracted from thermal 3D. Having this information throughout the daytime when the field is under stress gives insight about the field conditions.

In order to illustrate some of the analysis that can be performed with this $4 \mathrm{D}$ RGB model, in Figure 6.12(b) we removed the ground plane of the field at 19 DAP, leaving only the canopy temperatures. This important step of segmenting canopy from the soil is made trivial by filtering the point cloud based on their $\mathrm{Z}$ coordinates since the reference frame of the model is aligned with the ground. This is just one 


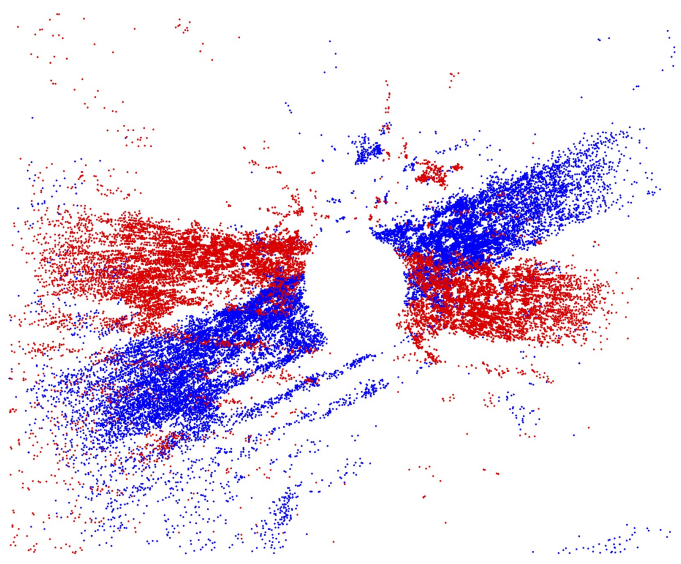

(a)

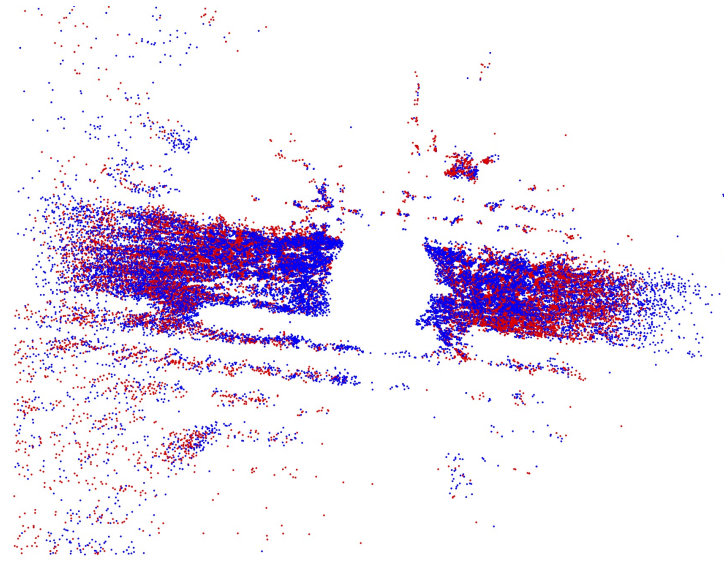

(b)

Figure 6.15: Temporal registration of two sparse point clouds created using images taken with 5 days time period gap (a) before optimization (b) after optimization

simple example of the potential information that can be obtained from the 4D-RGB model and used to study the behavior of different genotypes with respect to soil and air temperature, the effect of row spacing on different corn and sorghum hybrids, etc.

\subsubsection{D Reconstruction}

To perform temporal analysis of plants, all models should be registered in the same reference coordinate frame. This can be achieved by the registration of temporal models. Therefore, as mentioned in Chapter 5, one way to register these temporal models is by limiting the temporal constraints to be within a 5-day period (rigid), and solve the error propagation problem by constructing a virtual loop. The virtual loop is closed by two sets of frames used to go forward and backward in time.

Figure 6.15 shows qualitative results for the temporal registration step of the pipeline. As it can be inferred from the figure, there was a great improvement in the registration of two sets of 4D-RGB models before 6.15(a) and after 6.15 (b) the optimization. In Figure 6.15, for better qualitative illustration, only two point clouds from many temporal models registered together are shown. 


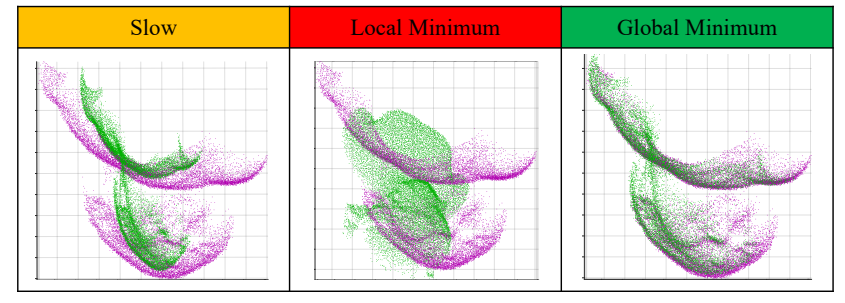

(a) Different convergence depending on $\mu$

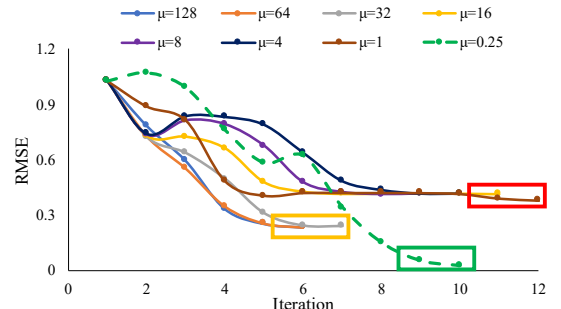

(b) The RMS error in each iteration

Figure 6.16: Non-rigid registration for different $\mu$ values of Geman-McClure robust function $\rho(x)=\frac{\mu x^{2}}{\mu+x^{2}}$

\subsection{Non-Isometric Registration}

In this section, the results for the non-isometric registration and optimization method introduced in Chapter 5 is presented. The proposed optimization method to register two clouds of points uses a non-isometric non-rigid transformation with rotation, translation, and scale parameters (total 9 parameters) along with the damped method.

\subsubsection{Synthetic range dataset}

As described before, the use of Geman-McClure robust estimator helps the optimization to automatically reject the outliers. However, the choice of the hyper-parameter $\mu$ of the estimator is not trivial as the optimal choice is varied depending on the data and the optimization problem. Figure 6.16b shows three different cases depending on the value of $\mu$. When the $\mu$ is too large $(\mu=128,64,32)$, the convergence becomes very slow and eventually stops in a local minimum of the robust estimator function while clearly it is not the local minimum for mean squared error objective function. Making the value of $\mu$ smaller $(\mu=16,8,4,1)$ makes the solution converged to another local minimum. Reducing the $\mu$ further $(\mu=0.25)$ we get a solution that converges to the global minimum.

Looking closer, we can find the optimal choice of $\mu$ as it is illustrated in Figure 6.17a. As can be seen, we varied the value of $\mu$ by increasing and decreasing it around $\mu=0.25$ until it failed. While $\mu=0.41$ was the optimal choice of $\mu$, both in terms of speed and accuracy, others tend to converge to the same level of accuracy 


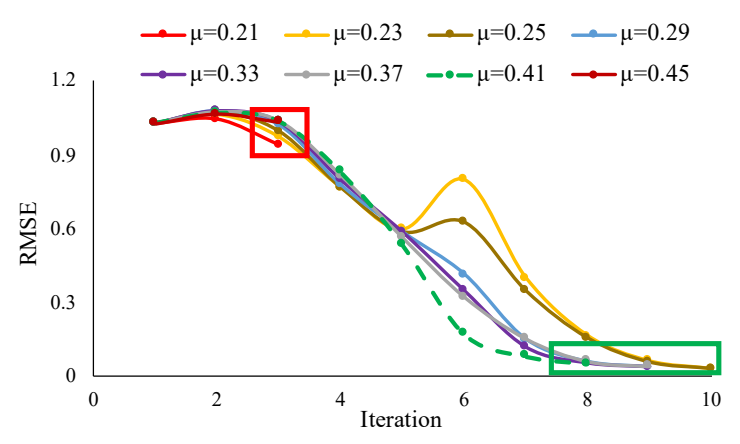

(a) The optimal choice of $\mu=0.41$.

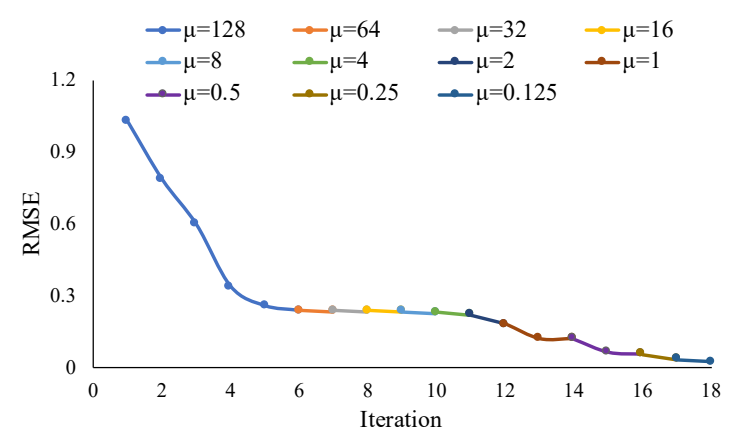

(b) Gradual convergence by decreasing $\mu$

Figure 6.17: Optimization of non-rigid registration using Geman-McClure robust estimator objective function

but took longer iterations by passing some potential local minimum. Therefore, in a none trivial approach, one could try a different value of $\mu$ and find the optimal choice in which optimization converges to the global minimum.

Another approach is to start with a larger value of $\mu$ and gradually decrease it until converged to the global minimum. Figure $6.17 \mathrm{~b}$ shows the RMS error of non-rigid registration while we reduce $\mu$ as we iterate. Using this technique, one can avoid the local minimum by distorting the hyper-surface of the objective function when reducing the $\mu$.

In order to illustrate the effect of Geman-McClure robust estimator objective function, we compared three objective functions: 1) with robust estimator penalty function, 2) without penalty function, 3) Fast Global Registration introduced in [1]. We used synthetic range data with the same correspondences in our experiments. In all experiments we used 6 parameters ( 3 rotation, and 3 translation), that is all three cases are solving a rigid registration problem. Figure 6.18 shows this experiment. As can be seen, the first case (objective function with penalty function solved with damped method while the value of $\mu$ is gradually reduced) obtained significantly better result compared to the vanilla objective function without a robust estimator. Our method works similarly to that of FGR [1] with the difference in the optimization approach. We use the damped method described in Chapter 5 and gradually decrease the $\mu$ in each optimization stage. Also, the FGR approximates the transformation by the differential motion which works fine for rigid registration. 


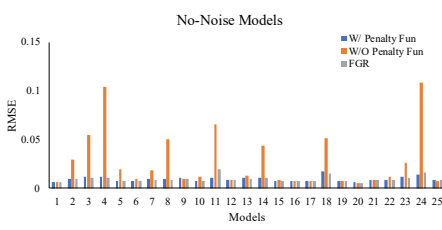

(a)

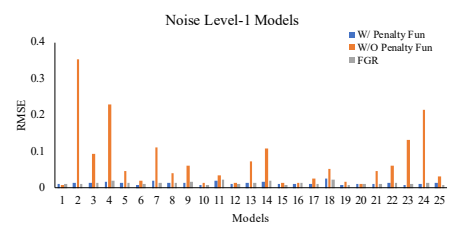

(b)

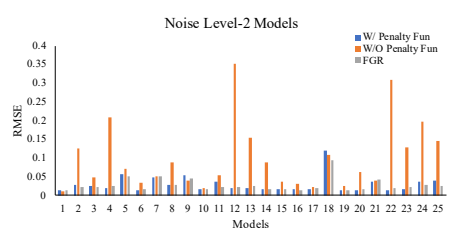

(c)

Figure 6.18: A comparison between objective functions defined (a) with penalty function, (b) without penalty function, (c) as described in fast global registration approach [1].

However, adding scale parameters to the approximation makes the optimization fail most of the time.

Figure 6.19 shows the qualitative results of our proposed non-rigid registration for sample models with no noise and noise level 2. Results for all 25 models converged to the global minimum with RMS error proportion to the noise level. The average RMS error for models with $\sigma=0,0.0025,0.005$ are $0.0102,0.0207,0.0562$, respectively. For all experiments, the source models were scaled with factors of $s_{x}=0.5, s_{y}=$ $0.5, s_{z}=0.5$.

\subsubsection{Non-Isometric Registration of African Violet dataset}

In this section, the result for applying the non-isometric registration method on the African violet dataset is described. Figure 6.20 shows the pipeline for spatiotemporal reconstruction of african violet using the dataset described in 6.1.3.

The pipeline starts with 3D reconstruction of images captured over 10 days. This step produces dense 3D point clouds of the plant at different timestamps (total 197 models). Next, a pre-processing stage is considered to reduce the size of models and extract 3D keypoints representing important regions of the model. These 3D keypoints are then used to extract 3D feature descriptors. Using the extracted feature descriptors, correspondences between temporal models are found. We then use a bundle registration algorithm to register temporal models with respect to a global coordinate frame. 


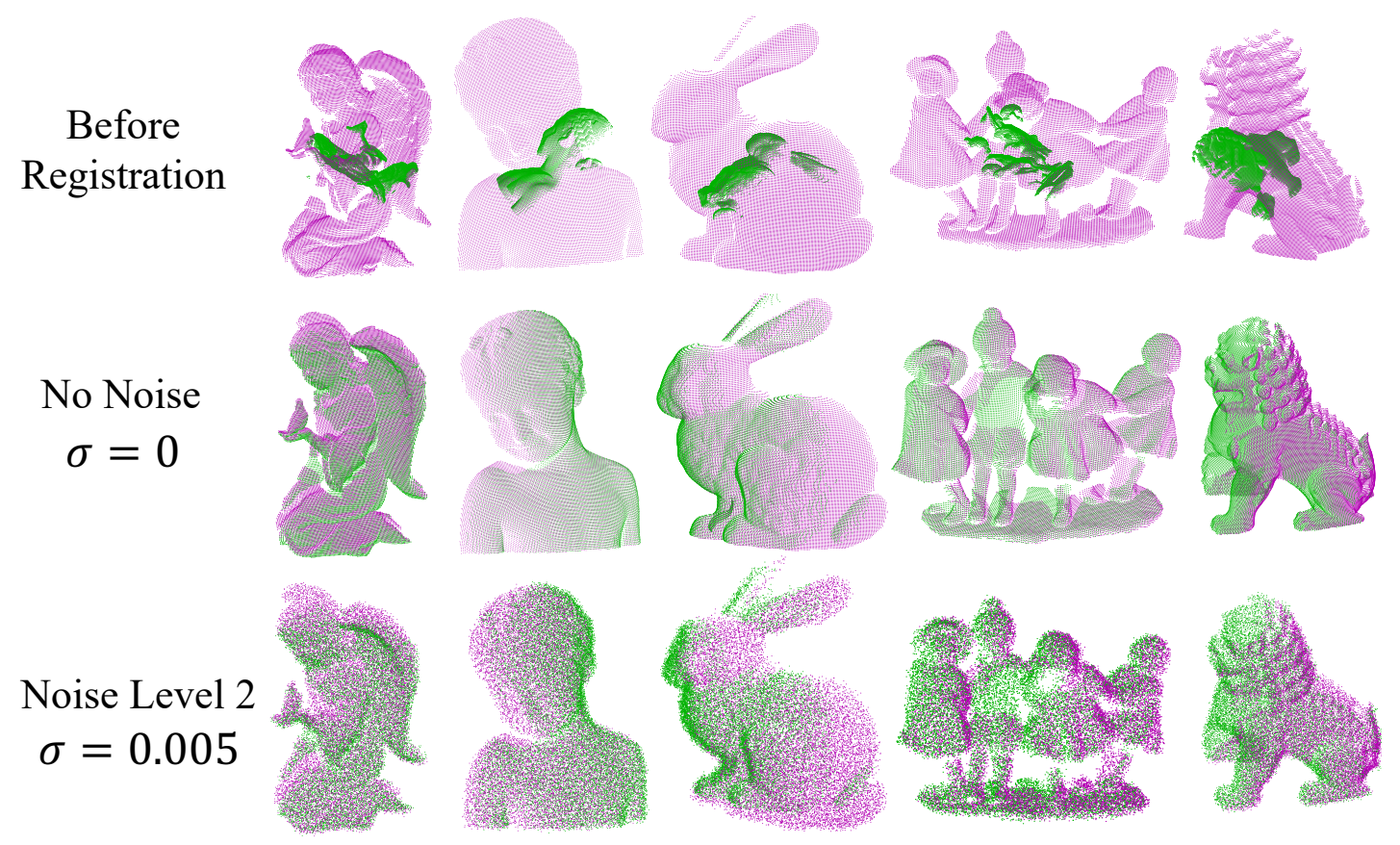

Figure 6.19: Qualitative results for non-rigid registration. Top row models show source and target point cloud before registration. Middle and bottom rows are the results of non-rigid registration for models with no noise and noise level 2, respectively.

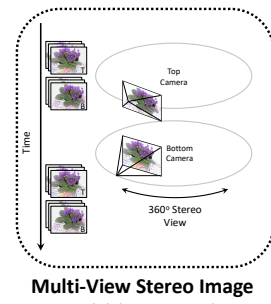
Acquisition Over Time
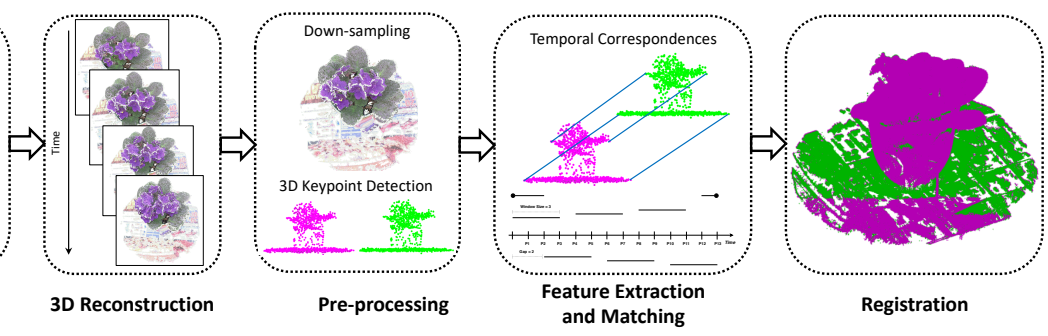

Figure 6.20: The pipeline for the proposed method of spatio-temporal reconstruction 


\subsection{Non-Isometric Registration}

\section{$3 \mathrm{D}$ reconstruction}

The COLMAP $[2,3]$ is used to process all the stereo images' sets and create 3D dense point clouds of the African violet plant. The COLMAP is a general-purpose Structure-from-Motion (SfM) and Multi-View Stereo (MVS) pipeline with a graphical user interface that offers a wide range of features for 3D reconstruction tasks from multiple image sets. The top and bottom images were separated as they had different intrinsic parameters. Although camera calibration of the dataset is available, in this research we let the COLMAP to estimate intrinsic and extrinsic camera parameters and as a result the output 3D models are in different scales. This is mainly to show effectiveness of the proposed algorithm for registration of models with different scales. The default parameters of COLMAP along with OpenCV camera model was used. In Figure 6.4, four typical reconstructed point clouds for days 1, 4, 7, and 10 captured at 1:00 AM are shown. In total, 197 point clouds of the plant over 10 days were reconstructed and provided with the publicly available dataset. The provided temporal models are all registered with respect to the same coordinate frame using the proposed registration method described next.

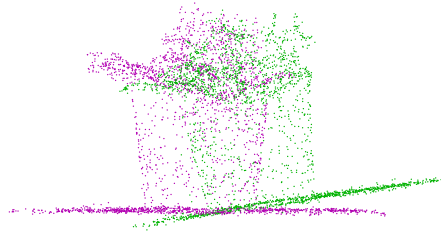

(a) FPFH

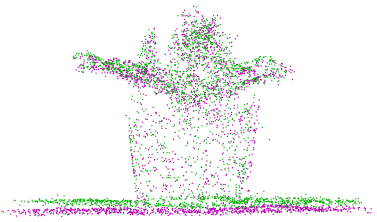

(b) PFHRGB

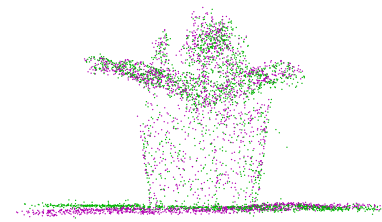

(c) $\mathrm{PFH}$

Figure 6.21: An example of the 3D registration of the same two consecutive point clouds from the violet dataset using (a) PFH, (b) FPFH, and (c) PFHRGB

\section{Preprocessing}

In this step, the original 3D point clouds are downsampled to speed up the computation. The leaf size of 0.01 is empirically chosen for the results reported in this work. Also 3D Harris keypoints are extracted to further speed-up the feature extraction and matching step. 


\subsection{Non-Isometric Registration}

\section{Feature extraction and Matching}

Looking at the literature, different 3D descriptors are introduced for different computer vision problems e.g. registration, seen recognition, object detection, etc [53]. Among these 3D feature descriptors, Point Feature Histogram (PFH) [54], Fast Point Feature Histogram (FPFH) [55] and Color Point Feature Histogram (PFHRGB) that was developed by the PCL community [56] are suited for 3D registration of point clouds. In that regard, a comprehensive review of 3D point cloud descriptors showed PFHRGB outperform other descriptors for our registration task. These feature descriptors are empirically evaluated on the entire violet dataset by varying the matching threshold, and PFHRGB which provided the best overall alignment results was retained in our methodology. Figure 6.21 shows a 3D registration example when trying to align the violet point clouds using different 3D feature descriptors mentioned above.

\section{Normalization}

As mentioned before, to show effectiveness of the registration, uncalibrated images used in COLMAP for reconstruction. As a result, COLMAP produced point clouds in different scales. As a preprocessing step, a normalization step is applied to help the registration discussed next. Given an input point cloud $P$ of size $3 \times N$ where the three dimensions represent $(X, Y, Z) 3 \mathrm{D}$ coordinates of the vertices, we normalized each of the point clouds by applying the following equations:

$$
\begin{aligned}
& \mu_{i}=\frac{\sum_{j=1}^{N} P_{i}(:, j)}{N} \\
& P_{0_{i}}=P_{i}-\mu_{i} \\
& s=\left\|P_{0_{i}}\right\| \\
& P_{n_{i}}=\frac{P_{i}-\mu_{i}}{s}
\end{aligned}
$$

where $N$ is the number of vertices, $\mu_{i}$ is the center of the point cloud $i$ and $s$ is the scale. $P$ and $P_{n}$ are respectively the unnormalized and normalized 3D point clouds.

\section{Bundle Registration}

A bundle registration approach is adopted to align all the temporal point clouds. At each optimization stage, we bundle temporal point clouds of a sliding window size, 


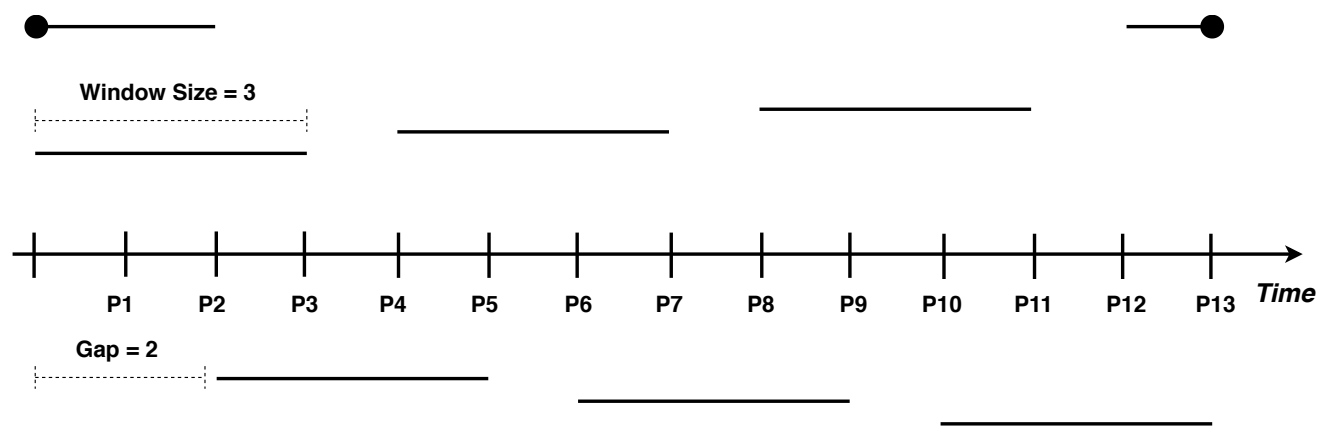

Figure 6.22: Representation of bundles with sliding window and gap. At each bundle optimization, all models within the bundle are aligned with respect to the first model. Sliding this bundle optimization by a gap step smaller than window size ensures overlaps.

fix the coordinate of the first point cloud, and estimate the transformations that best register rest of the clouds in the bundle. We then move to the next optimization bundle by a gap size. Bigger sliding window and smaller gap size are the optimal choice, but it comes with computation burdens. We empirically retained a window size of 10 (models) and a gap of 5 (models) for the results presented in this work. The optimization phase uses the point correspondences between models within the sliding window and minimizes the following objective function:

$$
J=\min _{r, t, s} \sum_{(i, j) \in \mathcal{P}} \rho\left(P_{j}-{ }^{w} H_{j}^{-1} \times{ }^{w} H_{i} \times P_{i}\right)
$$

where $P_{i}$ and $P_{j}$ are corresponding $3 \mathrm{D}$ points in source and target point clouds within the bundle. The indices $i$, and $j$ belong to all possible pairs $\mathcal{P}$ within the bundle. The parameters of the optimization: $r, t, s$, are the rotation, translation, and scale of each model with respect to global coordinate frame. At each optimization phase the first model parameters are excluded from the optimization leaving $(S W-1) \times D o F$ parameters for the optimization. The $\rho($.$) is a robust penalty function to reject$ outliers. The Graduated Non-Convexity (GNC) approach is used to solve the nonconvex optimization problem.

\section{Experimental Evaluations}

The evaluation of non-rigid registration methods is not straight-forward and visual assessments have been used over the years [57]. In this work, the experiments were designed to qualitatively and quantitatively evaluate the proposed spatio-temporal 


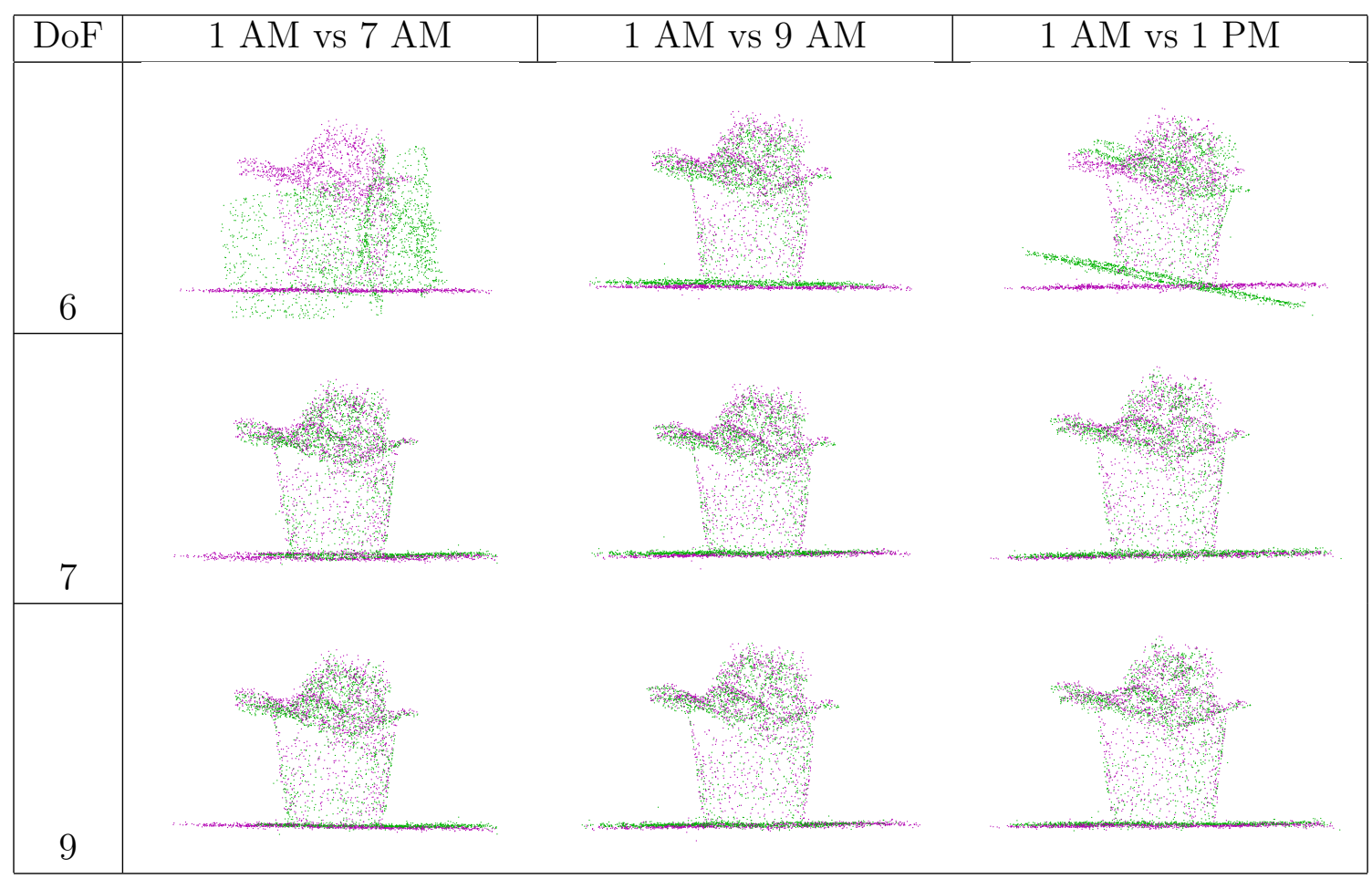

Figure 6.23: registration of violet dataset using the pairwise approach.

registration pipeline on the African violet dataset. For qualitative evaluation, we conduct a visual assessment of the registration results and also visualize the timeseries models using VisND [58].

For quantitative evaluation, we used the registration error between the source $P_{s}$ and target $P_{t}$ point clouds as defined in the following equation:

$$
E=\frac{1}{K} \sum_{k=1}^{K}\left\|p_{1}^{i}-p_{2}^{j}\right\|^{2}
$$

where $E$ is the residual, $K$ is the number of vertices in $P_{s}, p_{2}^{j}$ is thevertex in $P_{t}$ that is the closest to the vertex $p_{1}^{i}$ in $P_{s}$.

In order to show the performance of the proposed approach, the experiments were based on three approaches: pairwise, incremental and bundle registrations. For each approach, three types of transformations were evaluated under 6 (3 translations and 3 rotations), 7 (3 translations, 3 rotations and 1 scale) and 9DoF (3 translations, 3 rotations and 3 scales) to appreciate the effectiveness of the proposed approach in the presence of scale during the registration of time lapsed 3D point clouds. 


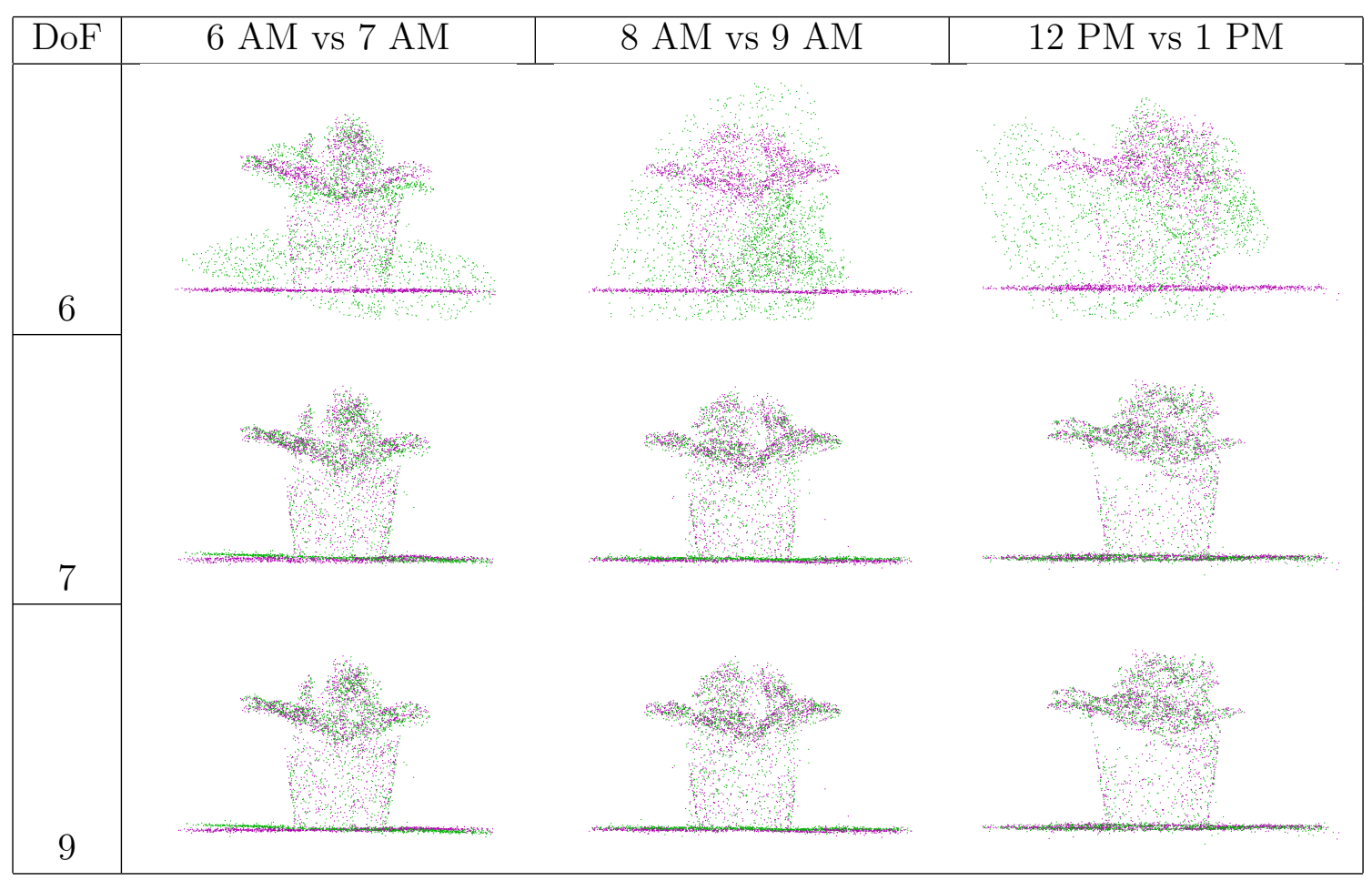

Figure 6.24: registration of violet dataset using the incremental approach.

\section{Pairwise registration}

A pairwise registration was applied to align all the temporal 3D models with respect to the first 3D Model generated in day 1 taken as the global coordinate frame. Figure 6.23 illustrates the results of the pairwise registration for three example pairs of point clouds under 6, 7 and 9 DoF transformations.

\section{Incremental registration}

In this approach, consecutive pairs of point clouds were registered at a time. By incrementally registering the models, we propagating the transformations from the initial to the final models. The downside of this method is that the error also propagates throughout the temporal registration when more models were added. Figure 6.24 illustrates the results of the incremental registration for three example pairs of point clouds under 6, 7 and 9 DoF transformations. 


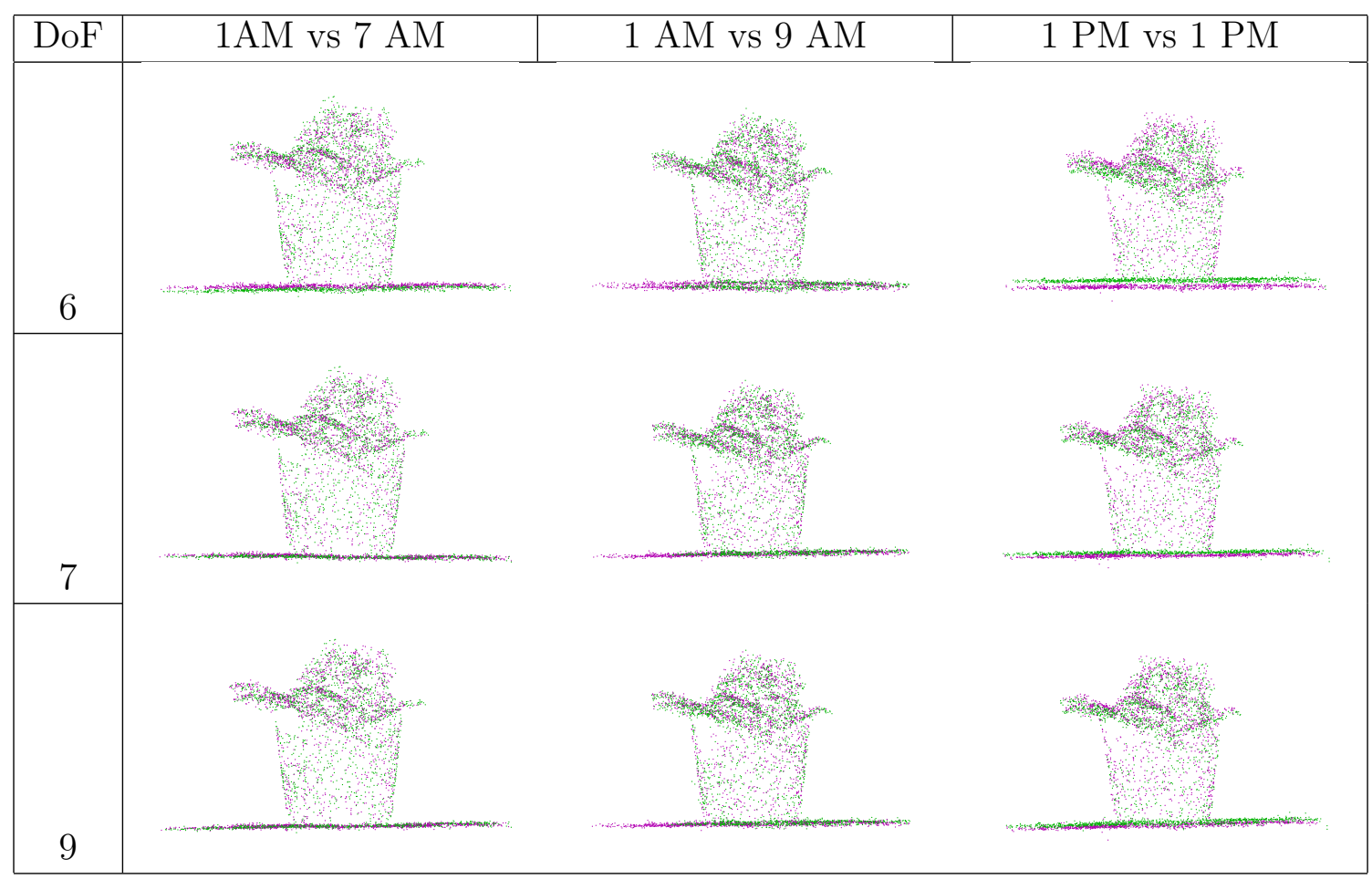

Figure 6.25: registration of violet dataset using the bundle approach.

Table 6.2: Average errors for each time-series registration approach with theAfrican violet dataset under 6,7 and 9 DoF transformations. The values in the table are expressed in millimeter $(\mathrm{mm})$

\begin{tabular}{cccc}
\hline Approach & 6 DoF & 7 DoF & 9 DoF \\
\hline \hline Pairwise & 10.7459 & 1.5951 & 1.5878 \\
Incremental & 36.7156 & 3.7012 & 3.7856 \\
Bundle & $\mathbf{1 . 7 3 6 4}$ & $\mathbf{1 . 5 7 7 8}$ & $\mathbf{1 . 5 8 6 2}$ \\
\hline
\end{tabular}

\section{Bundle registration}

A window size of 10 and a gap size of 5 are empirically retained for the results presented here. The bundle registration is able to handle the presence of noise, outliers and different levels of overlaps in the 3D point clouds as shown in Figure 6.25 .

In Table 6.2, the average registration errors for all approaches over the entire African violet dataset is reported . In Figure 6.26, the average registration errors are compared over ten days. We obtained an average error of less than $2 \mathrm{~mm}$ with using the proposed bundle registration approach, which indicates the effectiveness of the method. 


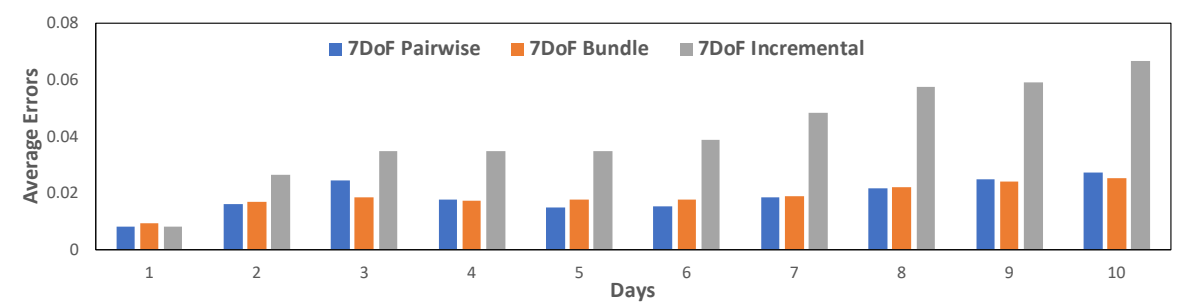

Figure 6.26: A comparison between all the registration methods over 10 days under the 7 DoF transformation

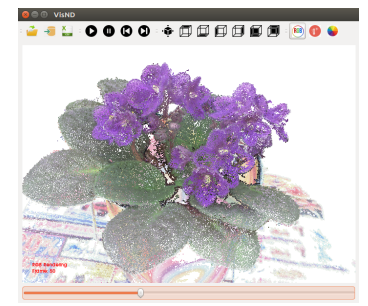

(a) Frame 50

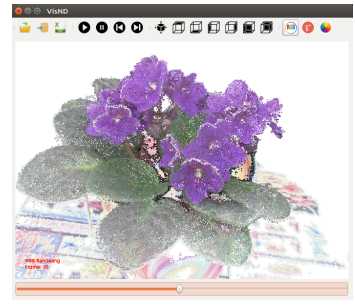

(b) Frame 70

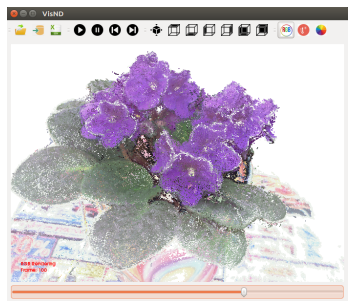

(c) Frame 100

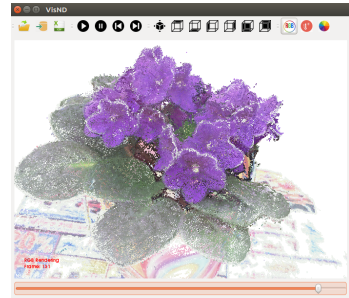

(d) Frame 130

Figure 6.27: Snapshots of VisND with Spatio-temporal models of African violet

\section{Qualitative Analysis}

The VisND [58], a multidimensional visualization tool for plant phenotyping is used to visualize the aligned spatio-temporal 3D models. The tool allows to effectively visualize the changes occurring during the plant development over time by playing sequences of aligned time-series point clouds as a video. In addition, the point cloud can be manipulated to inspect and get additional information about the plant at each specific frame (Figure 6.27). 


\section{Chapter 7}

\section{Conclusions and Future Work}

In the agriculture and food industries, the use of technology is rapidly growing to improve food production both in quantity and quality. Imaging technology can greatly influence the industry by enabling researchers to measure important aspects. The fusion of plant data into a 3D model of the canopy enables and eases plant phenotyping where the correspondence between plants and collected data is of great importance to researchers. Combination of RGB, Thermal and 3D model over the growing season makes the interpretation of plant status in the context of environmental conditions easier (e.g. the correlation between growth rate with average soil or plant temperature over the growing season).

Our proposed phenotyping platforms can collect multi-modal spatio-temporal information from plants and process the raw data and create unified and consistent data that can be used to extract phenotyping features. Specifically, our 5D (3D model with temperature over time) model of the field is capable of providing rich information such that many plant phenotypes can be extracted and correlated with each other and with plant performance. The spatio-temporal reconstruction pipeline is as follows: first 3D models of the canopy at different timestamps are reconstructed, next, temperature information is fused with the reconstructed 3D model and finally, all individual temporal models are registered to the same reference coordinate frame. We also proposed a non-isometric registration algorithm with rotation, translation, and scale (total 9 parameters) as an alternative for the last stage of the pipeline. The results showed the effectiveness of the robust estimator in objective function of 


\subsection{Future work}

the optimization.

While adding multiple dimensions to the data can help plant phenotyping, analyzing these data can become overwhelming, error-prone, and time consuming for humans. Therefore, fusing, cropping, detailing, and expanding the data help plant scientists to analyze, correlate, and infer new information from the data. Effective visualization techniques applied to these high dimensional models can provide the required tool for better research. Therefore, we introduced a multidimensional visualization tool (VisND) that can render a series of 4D-RGB point clouds according to the user's choices of viewing points. The software provides a useful tool for the user to analyze the spatio-temporal model of the canopy.

\subsection{Future work}

The proposed method for non-rigid registration has shown to be effective for data where the deformation is small and incremental. However, in some cases with large deformation, the algorithm is less effective since deformation cannot be handle by non-isometric transformation. In such cases a variant of deformation graph is proposed to solve the non-rigid registration problem for deformable objects. In that regard, a patched base deformation graph can be used to register a source model to a target model. The patched based deformation graph can have less complexity as the original deformation graph. 


\section{Bibliography}

[1] Q.-Y. Zhou, J. Park, and V. Koltun, "Fast global registration," in European Conference on Computer Vision. Springer, 2016, pp. 766-782. (document), $5.5,6.1 .2,6.3 .1,6.18$

[2] J. L. Schönberger, E. Zheng, M. Pollefeys, and J.-M. Frahm, "Pixelwise view selection for unstructured multi-view stereo," in European Conference on Computer Vision (ECCV), 2016. (document), 6.4, 6.3.2

[3] J. L. Schönberger and J.-M. Frahm, "Structure-from-motion revisited," in Conference on Computer Vision and Pattern Recognition (CVPR), 2016. (document), $6.4,6.3 .2$

[4] S. C. Chapman, T. Merz, A. Chan, P. Jackway, S. Hrabar, M. F. Dreccer, E. Holland, B. Zheng, T. J. Ling, and J. Jimenez-Berni, "Pheno-copter: a lowaltitude, autonomous remote-sensing robotic helicopter for high-throughput field-based phenotyping," Agronomy, vol. 4, no. 2, pp. 279-301, 2014. (document), 2.1, 2.1, ??, 2.3.3

[5] G. Fischer, "World food and agriculture to 2030/50," in Technical paper from the Expert Meeting on How to Feed the World in, vol. 2050, 2009, pp. 24-26. 2

[6] M. M. Chaves, J. P. Maroco, and J. S. Pereira, "Understanding plant responses to drought-from genes to the whole plant," Functional plant biology, vol. 30, no. 3, pp. 239-264, 2003. 2

[7] J. L. Araus and J. E. Cairns, "Field high-throughput phenotyping: the new crop breeding frontier," Trends in Plant Science, vol. 19, no. 1, pp. 52-61, 2014. 2 
[8] F. Fiorani and U. Schurr, "Future scenarios for plant phenotyping," Annual review of plant biology, vol. 64, pp. 267-291, 2013. 2

[9] P. L. Gregersen, A. Culetic, L. Boschian, and K. Krupinska, "Plant senescence and crop productivity," Plant Molecular Biology, vol. 82, no. 6, pp. 603-622, 2013. 2.1

[10] M. B. Cruzan, B. G. Weinstein, M. R. Grasty, B. F. Kohrn, E. C. Hendrickson, T. M. Arredondo, and P. G. Thompson, "Small unmanned aerial vehicles (micro-uavs, drones) in plant ecology," Applications in Plant Sciences, vol. 4, no. 9 , p. $1600041,2016.2 .1$

[11] D. Anthony, S. Elbaum, A. Lorenz, and C. Detweiler, "On crop height estimation with uavs," in Intelligent Robots and Systems (IROS 2014), 2014 IEEE/RSJ International Conference on. IEEE, 2014, pp. 4805-4812. 2.1

[12] S. Tisne, Y. Serrand, L. Bach, E. Gilbault, R. Ben Ameur, H. Balasse, R. Voisin, D. Bouchez, M. Durand-Tardif, P. Guerche et al., "Phenoscope: an automated large-scale phenotyping platform offering high spatial homogeneity," The Plant Journal, vol. 74, no. 3, pp. 534-544, 2013. 2.1, 2.2, ??

[13] P. Andrade-Sanchez, M. A. Gore, J. T. Heun, K. R. Thorp, A. E. Carmo-Silva, A. N. French, M. E. Salvucci, and J. W. White, "Development and evaluation of a field-based high-throughput phenotyping platform," Functional Plant Biology, vol. 41, no. 1, pp. 68-79, 2014. 2.1, ??, 2.3.3

[14] J. Barker, N. Zhang, J. Sharon, R. Steeves, X. Wang, Y. Wei, and J. Poland, "Development of a field-based high-throughput mobile phenotyping platform," Computers and Electronics in Agriculture, vol. 122, pp. 74-85, 2016. 2.1

[15] C. Y. Chen, C. L. Butts, P. M. Dang, and M. L. Wang, "Advances in phenotyping of functional traits," in Phenomics in Crop Plants: Trends, Options and Limitations. Springer, 2015, pp. 163-180. 2.1, ??

[16] P. S. Basu, M. Srivastava, P. Singh, P. Porwal, R. Kant, and J. Singh, "Highprecision phenotyping under controlled versus natural environments," in Phe- 
nomics in Crop Plants: Trends, Options and Limitations. Springer, 2015, pp. 27-40. 2.1, 2.2

[17] K. H. von Mogel, "Phenomics revolution," 2013. 2.1

[18] J. L. Araus, G. A. Slafer, C. Royo, and M. D. Serret, "Breeding for yield potential and stress adaptation in cereals," Critical Reviews in Plant Science, vol. 27 , no. 6 , pp. $377-412,2008.2 .1$

[19] L. Busemeyer, D. Mentrup, K. Möller, E. Wunder, K. Alheit, V. Hahn, H. P. Maurer, J. C. Reif, T. Würschum, J. Müller et al., "Breedvision-a multi-sensor platform for non-destructive field-based phenotyping in plant breeding," Sensors, vol. 13, no. 3, pp. 2830-2847, 2013. 2.1

[20] A. Ruckelshausen, P. Biber, M. Dorna, H. Gremmes, R. Klose, A. Linz, F. Rahe, R. Resch, M. Thiel, D. Trautz et al., "Bonirob-an autonomous field robot platform for individual plant phenotyping," Precision agriculture, vol. 9, no. 841, p. 1, 2009. 2.1

[21] B. Åstrand and A.-J. Baerveldt, "A vision based row-following system for agricultural field machinery," Mechatronics, vol. 15, no. 2, pp. 251-269, 2005. 2.1

[22] D. Deery, J. Jimenez-Berni, H. Jones, X. Sirault, and R. Furbank, "Proximal remote sensing buggies and potential applications for field-based phenotyping," Agronomy, vol. 4, no. 3, pp. 349-379, 2014. 2.1

[23] M. Hamza and W. Anderson, "Soil compaction in cropping systems: A review of the nature, causes and possible solutions," Soil and tillage research, vol. 82, no. 2 , pp. 121-145, 2005. 2.1

[24] E. Yol, C. Toker, and B. Uzun, "Traits for phenotyping," in Phenomics in Crop Plants: Trends, Options and Limitations. Springer, 2015, pp. 11-26. 2.2

[25] N. Fahlgren, M. A. Gehan, and I. Baxter, "Lights, camera, action: highthroughput plant phenotyping is ready for a close-up," Current opinion in plant biology, vol. 24, pp. 93-99, 2015. 2.2, 2.3.3 
[26] A. Ruckelshausen and L. Busemeyer, "Toward digital and image-based phenotyping," in Phenomics in Crop Plants: Trends, Options and Limitations. Springer, 2015, pp. 41-60. 2.2

[27] D. Rousseau, H. Dee, and T. Pridmore, "Imaging methods for phenotyping of plant traits," in Phenomics in Crop Plants: Trends, Options and Limitations. Springer, 2015, pp. 61-74. 2.2

[28] C. L. McCarthy, N. H. Hancock, and S. R. Raine, "Applied machine vision of plants: a review with implications for field deployment in automated farming operations," Intelligent Service Robotics, vol. 3, no. 4, pp. 209-217, 2010. 2.2

[29] "Scnalyzer 3d high throughput," URL: http://www.lemnatec.com/products/hardware-solutions/scanalyzer-3d-high-throughput, 2016. 2.2

[30] C. N. Topp, A. S. Iyer-Pascuzzi, J. T. Anderson, C.-R. Lee, P. R. Zurek, O. Symonova, Y. Zheng, A. Bucksch, Y. Mileyko, T. Galkovskyi et al., "3d phenotyping and quantitative trait locus mapping identify core regions of the rice genome controlling root architecture," Proceedings of the National Academy of Sciences, vol. 110, no. 18, pp. E1695-E1704, 2013. 2.2

[31] T. K. D. Nakini and G. N. DeSouza, "Distortion correction in 3d-modeling of root systems for plant phenotyping," in Computer Vision-ECCV 2014 Workshops. Springer, 2014, pp. 140-157. 2.2

[32] M. A. Piñeros, B. G. Larson, J. E. Shaff, D. J. Schneider, A. X. Falcão, L. Yuan, R. T. Clark, E. J. Craft, T. W. Davis, P.-L. Pradier et al., "Evolving technologies for growing, imaging and analyzing $3 \mathrm{~d}$ root system architecture of crop plants," Journal of integrative plant biology, vol. 58, no. 3, pp. 230-241, 2016. 2.2

[33] S. Jay, G. Rabatel, X. Hadoux, D. Moura, and N. Gorretta, "In-field crop row phenotyping from 3d modeling performed using structure from motion," Computers and Electronics in Agriculture, vol. 110, pp. 70-77, 2015. 2.2 
[34] A. Shafiekhani, S. Kadam, F. B. Fritschi, and G. N. DeSouza, "Vinobot and vinoculer: Two robotic platforms for high-throughput field phenotyping," Sensors, vol. 17, no. 1, p. 214, 2017. 2.3

[35] A. Shafiekhani, F. B. Fritschi, and G. N. DeSouza, "Vinobot and vinoculer: from real to simulated platforms," in Autonomous Air and Ground Sensing Systems for Agricultural Optimization and Phenotyping III, vol. 10664. International Society for Optics and Photonics, 2018, p. 106640A. 2.3

[36] N. Virlet, K. Sabermanesh, P. Sadeghi-Tehran, and M. J. Hawkesford, "Field scanalyzer: An automated robotic field phenotyping platform for detailed crop monitoring," Functional Plant Biology, 2017. ??, 2.3.3

[37] S. Choi, Q.-Y. Zhou, and V. Koltun, "Robust reconstruction of indoor scenes," in Proceedings of the IEEE Conference on Computer Vision and Pattern Recognition, 2015, pp. 5556-5565. 4.1

[38] N. Gelfand, N. J. Mitra, L. J. Guibas, and H. Pottmann, "Robust global registration," in Symposium on geometry processing, vol. 2, no. 3. Vienna, Austria, 2005, p. 5. 4.1

[39] D. Holz, A. E. Ichim, F. Tombari, R. B. Rusu, and S. Behnke, "Registration with the point cloud library: A modular framework for aligning in 3-d," IEEE Robotics \& Automation Magazine, vol. 22, no. 4, pp. 110-124, 2015. 4.1

[40] F. Pomerleau, F. Colas, R. Siegwart, and S. Magnenat, "Comparing icp variants on real-world data sets," Autonomous Robots, vol. 34, no. 3, pp. 133-148, 2013. 4.1

[41] S. Rusinkiewicz and M. Levoy, "Efficient variants of the icp algorithm," in Proceedings Third International Conference on 3-D Digital Imaging and Modeling. IEEE, 2001, pp. 145-152. 4.1

[42] S. Thrun, "Probabilistic robotics," Communications of the ACM, vol. 45, no. 3, pp. $52-57,2002.4 .1$ 
[43] G. Grisetti, R. Kummerle, C. Stachniss, and W. Burgard, "A tutorial on graphbased slam," IEEE Intelligent Transportation Systems Magazine, vol. 2, no. 4, pp. 31-43, 2010. 4.2.2

[44] Z. Zhang, "A flexible new technique for camera calibration," IEEE Transactions on pattern analysis and machine intelligence, vol. 22, no. 11, pp. 1330-1334, 2000. 5.1.1

[45] K. Strobl, W. Sepp, S. Fuchs, C. Paredes, and K. Arbter, "Camera calibration toolbox for matlab," Pasadena, CA, 2006. 5.1.1

[46] G. N. DeSouza, A. H. Jones, and A. C. Kak, "An world-independent approach for the calibration of mobile robotics active stereo heads," in IEEE International Conference on Robotics and Automation (ICRA), vol. 4. IEEE, 2002, pp. 33363341. 5.1.3, 5.2.1

[47] A. Shafiekhani, F. B. Fritschi, and G. N. DeSouza, "A new 4d-rgb mapping technique for field-based high-throughput phenotyping," in Computer Vision Problems in Plant Phenotyping. BMVC, 2018, pp. 1-13. 5.2, 5.2.1

[48] D. G. Lowe, "Object recognition from local scale-invariant features," in IEEE international conference on Computer vision, vol. 2. IEEE, 1999, pp. 11501157. 1

[49] Y. Furukawa and J. Ponce, "Accurate, dense, and robust multiview stereopsis," IEEE transactions on pattern analysis and machine intelligence, vol. 32, no. 8, pp. 1362-1376, 2010. 5

[50] J. Liu and E. Pattey, "Retrieval of leaf area index from top-of-canopy digital photography over agricultural crops," Agricultural and Forest Meteorology, vol. 150, no. 11, pp. 1485-1490, 2010. 5.2.4

[51] R. Kolluri, J. R. Shewchuk, and J. F. O'Brien, "Spectral surface reconstruction from noisy point clouds," in Proceedings of the 2004 Eurographics/ACM SIGGRAPH symposium on Geometry processing, 2004, pp. 11-21. 6.1.2 
[52] J. Goudriaan, "The bare bones of leaf-angle distribution in radiation models for canopy photosynthesis and energy exchange," Agricultural and forest meteorology, vol. 43, no. 2, pp. 155-169, 1988. 6.2.2

[53] L. A. Alexandre, "3d descriptors for object and category recognition: a comparative evaluation," in Workshop on Color-Depth Camera Fusion in Robotics at the IEEE/RSJ International Conference on Intelligent Robots and Systems (IROS), Vilamoura, Portugal, vol. 1, no. 3, 2012, p. 7. 6.3 .2

[54] R. B. Rusu, N. Blodow, Z. C. Marton, and M. Beetz, "Aligning point cloud views using persistent feature histograms," in 2008 IEEE/RSJ international conference on intelligent robots and systems. IEEE, 2008, pp. 3384-3391. 6.3 .2

[55] R. B. Rusu, N. Blodow, and M. Beetz, "Fast point feature histograms (fpfh) for 3d registration," in 2009 IEEE international conference on robotics and automation. IEEE, 2009, pp. 3212-3217. 6.3.2

[56] R. B. Rusu and S. Cousins, "3d is here: Point cloud library (pcl)," in 2011 IEEE international conference on robotics and automation. IEEE, 2011, pp. 1-4. 6.3 .2

[57] G. K. Tam, Z.-Q. Cheng, Y.-K. Lai, F. C. Langbein, Y. Liu, D. Marshall, R. R. Martin, X.-F. Sun, and P. L. Rosin, "Registration of 3d point clouds and meshes: A survey from rigid to nonrigid," IEEE transactions on visualization and computer graphics, vol. 19, no. 7, pp. 1199-1217, 2012. 6.3 .2

[58] A. Shafiekhani, F. B. Fritschi, and G. N. DeSouza, "Visnd: A visualization tool for multidimensional model of canopy," in Proceedings of the IEEE Conference on Computer Vision and Pattern Recognition Workshops, 2019, pp. 0-0. 6.3.2, 6.3 .2 


\section{Vita}

Ali Shafiekhani was born in Tehran, Iran. After earning his high school diploma in 2007, he attended the University of Kashan where he got his B.Sc. in Mechanical Engineering in 2011. He continued his graduate studies in Mechanical Engineering and received his M.Sc. from the University of Tehran in 2014 while serving as a Teaching Assistant in different undergraduate courses. During his M.Sc., Ali worked on intersections of Artificial Intelligence (AI), System Dynamics and Control, and Robotics. In 2014, Ali got admitted to the doctoral program at the Electrical Engineering and Computer Science Department, University of Missouri-Columbia. He started working as a research assistant in Vision-Guided and Intelligent Robotics Lab (ViGIR-Lab) developing new robotics platforms for plant phenotyping in the agricultural field. As a result, his research obtained media coverage reaching an officially estimated audience of over 1.5 million people in the US alone. These were through more than 20 radio and TV stations as well as various websites (e.g. WIRED, TechCrunch, EurekaAlert, etc.). While studying towards his Ph.D., Ali also served as the lab instructor for undergraduate and graduate-level robotics courses. In 2017, he received a second master's degree (M.E.) from Electrical Engineering Department made him a scientist with multi-disciplinary skills in Mechanical Engineering, Electrical Engineering, and Computer Science. In 2018, he was admitted to a summer school at the Massachusetts Institute of Technology (MIT) on Cognitive Robotics. He spent the summer of 2019 as a research intern at Philips Research North America conducting research on the application of Artificial Intelligence (AI) in healthcare. Ali received his Ph.D. degree in Electrical and Computer Engineering from the University of Missouri-Columbia in 2020. Over his academic journey, he has served as the reviewer and guest editor for top conferences and journals in his area of research. 